CSDL-T-1183

FUZZY BASED ATTITUDE CONTROLLER FOR FLEXIBLE SPACECRAFT WITH ON/OFF THRUSTERS

by

Roger Glenn Knapp

Mav 1993

Master of Science Thesis

Massachusetts Institute of Technology

(NASA-CR-188250) FUZZY BASED
ATTITUDE CONTROLLER FOR FLEXIBLE
SPACECRAFT WITH ON/OFF THRUSTERS
M.S. Thesis - M.I.T. 1993 (Draper
(Charles Stark) Lab.) $135 \mathrm{p}$

N93-32228

Unclas

G3/18 0176380

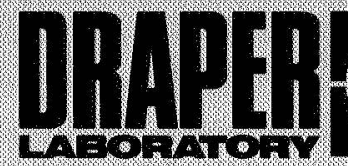

The Charles Stark Draper Laboratory inc: 
Fuzzy Based Attitude Controller for Flexible Spacecraft with On/Off Thrusters

by

\section{Roger Glenn Knapp}

S.B., Aeronautical and Astronautical Engineering Massachusetts Institute of Technology, Cambridge, MA

(1991)

\section{SUBMITTED TO THE DEPARTMENT OF AERONAUTICS AND ASTRONAUTICS IN PARTIAL FULFILLMENT \\ OF THE REQUIREMENTS FOR THE DEGREE OF}

\section{MASTER OF SCIENCE}

at the

\section{MASSACHUSETTS INSTITUTE OF TECHNOLOGY}

May, 1993

(C) 1993 Roger Glenn Knapp

All Rights Reserved

Signature of Author Dogen $\frac{\text { Department of Aeronautics \& Astronautics }}{20}$ May, 1993

Approved by

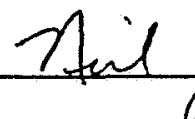
adarz Technical Staff, Charles Stark Draper Laboratory Neil J. Adams Technical Supervisor Certified by W allace $\sum$ Wach Welde Professor Wallace E. Vander Velde Thesis Supervisor

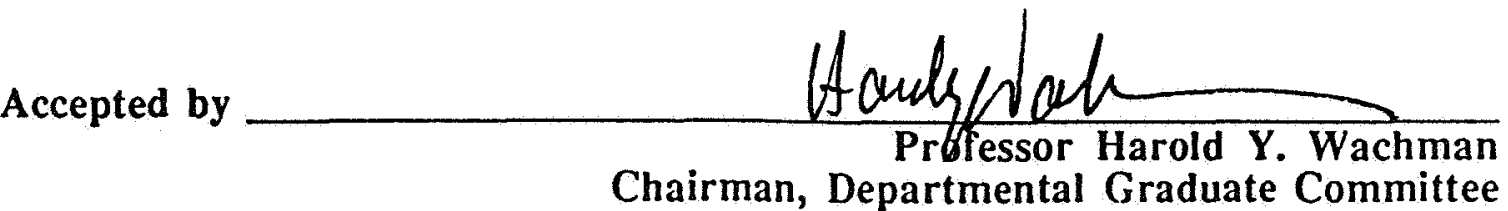





\title{
Fuzzy Based Attitude Controller for Flexible Spacecraft with On/Off Thrusters
}

\author{
by \\ Roger Glenn Knapp \\ Submitted to the Department of Aeronautics and \\ Astronautics on May 7, 1993 in partial fulfillment of the \\ requirements for the degree of Master of Science
}

\begin{abstract}
A fuzzy-based attitude controller is designed for attitude control of a generic spacecraft with on/off thrusters. The controller is comprised of packages of rules dedicated to addressing different objectives (e.g., disturbance rejection, low fuel consumption, avoiding the excitation of flexible appendages, etc.). These rule packages can be inserted or removed depending on the requirements of the particular spacecraft and are parameterized based on vehicle parameters such as inertia or operational parameters such as the maneuvering rate. Individual rule packages can be "weighted" relative to each other to emphasize the importance of one objective relative to another. Finally, the fuzzy controller and rule packages are demonstrated using the high-fidelity Space Shuttle Interactive On-Orbit Simulator (IOS) while performing typical on-orbit operations and are subsequently compared with the existing shuttle flight control system performance.
\end{abstract}

Technical Supervisor: Neil J. Adams

Technical Staff, Manned Space Systems Division

The Charles Stark Draper Laboratory, Inc.

Thesis Supervisor: $\quad$ Dr. Wallace E. Vander Velde

Professor of Aeronautics and Astronautics 



\section{Acknowledgments}

I would like to thank the Charles Stark Draper Laboratory for giving me the opportunity to pursue my Masters Degree at MIT, and John Sweeney and Joan Chiffer for making the Draper Fellow program possible.

Thanks also go to my thesis supervisors Neil Adams and Professor Vander Velde. Neil provided many insights into the operation of the space shuttle flight control system and ways to improve upon it. When the chips were down he transformed into a blinding reading machine, providing such detailed suggestions that I personally was quite amazed he could think them all up (Must have required a superhuman amount of patience!!). Thanks Professor Vander Velde for many valuable suggestions and your careful reading.

Ching, my officemate, deserves thanks for the first paragraph of these acknowledgments. More than that, she blazed the way through much of the meatloaf we must crawl through in pursuit of our degrees at MIT, making it much easier for the rest of us. Most of all, she pointed out to me one Friday that we had Monday off. Without her insight I would have missed a weekend with Kimi in Seattle. Thank you Ching.

Thanks to Jeff and Steve for ensuring I didn't work too hard on the weekends. Mountain biking in the Fells and New Hampshire, wingen on the lake. I'll miss that, but maybe you guys can come out West and trek down the slopes of some real mountains.

Thank you Kimi for all the late night calls that kept me going, and providing me with the incentive to finish this thesis pronto. Thanks Mom and Dad for instilling within me what it took to get to this place and time. 

This report was prepared at the Charles Stark Draper Laboratory, Inc. under contract NAS9-18147

Publication of this report does not constitute approval by Draper Laboratory or the sponsoring agency of the findings or conclusions contained herein. It is published solely for the exchange and stimulation of ideas.

I hereby assign my copyright of this thesis to the Charles Stark Draper Laboratory, Inc., Cambridge, Massachusetts.

\section{Roger Glenn Knapp}

Permission is hereby granted by the Charles Stark Draper Laboratory, Inc., to the Massachusetts Institute of Technology to reproduce any or all of this thesis. 



\section{Table of Contents}

Chapter 1 Introduction.....................................................

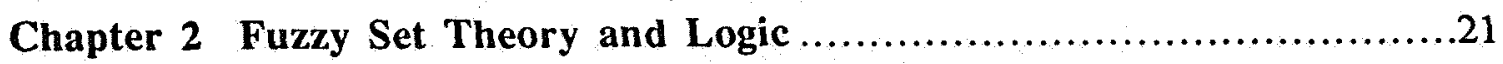

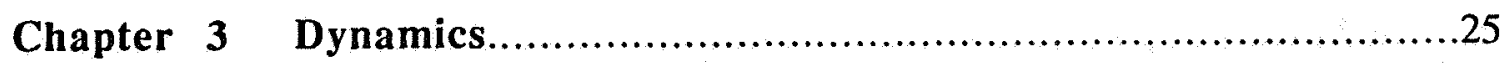

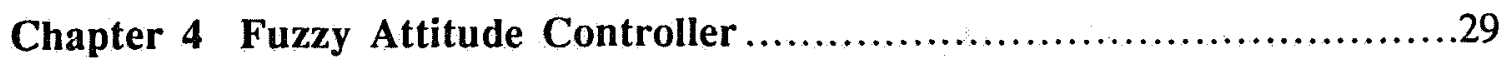

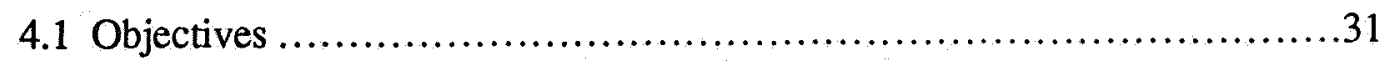

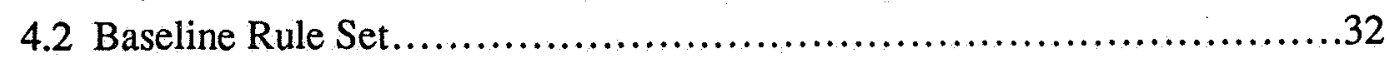

4.3 Supplemental Rule Packages....................................................37

4.4 Slow Disturbances Rule Package.................................................37

4.5 Jet Limiting Rule Package for Fuel Savings .............................44

4.6 Anti-Chatter Rule Package ...........................................45

4.7 Anti-Flex Rule Package.......................................................46

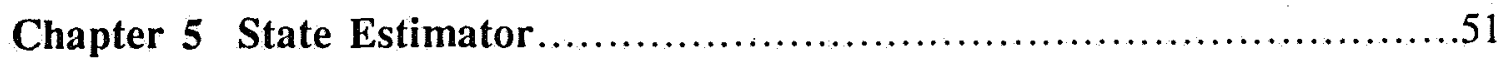

5.1 Slow Disturbance Acceleration Estimation ................................51

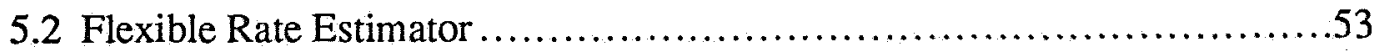

Chapter 6 Space Shuttle Characteristics...................................59

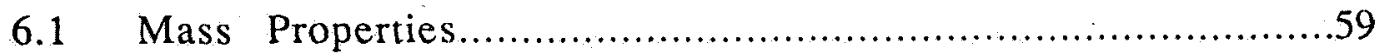

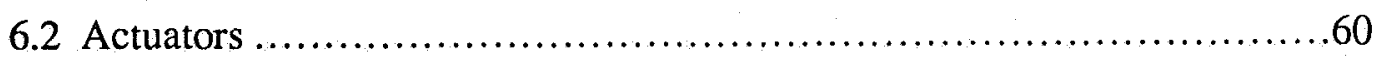

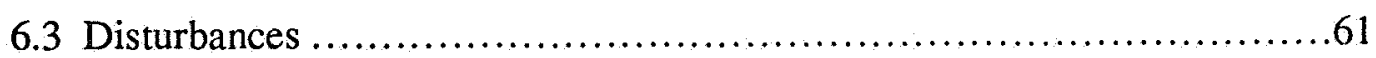

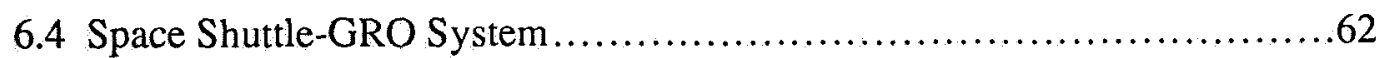

6.4.1 Combined Mass Properties .......................................62

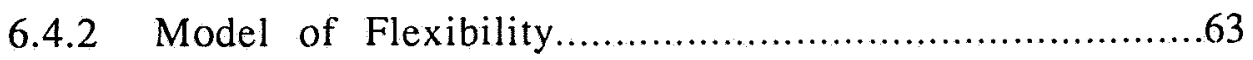

6.5 Current Shuttle Flight Control System.........................................63

6.5.1 Universal Pointing Module ...................................64

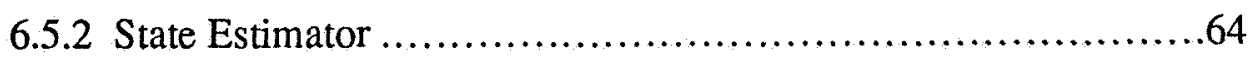

6.5.3 Auto Maneuver Module ...........................................65

6.5.4 Phase Plane Modules..............................................66

6.5.5 Jet Select Module ............................................69

6.5.5.1 Alt Mode.....................................................70

6.5.6 Modifications to Space Shuttle Flight Control System ............70 


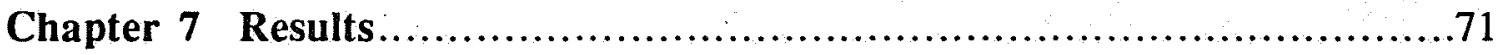

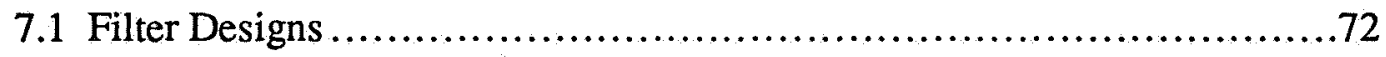

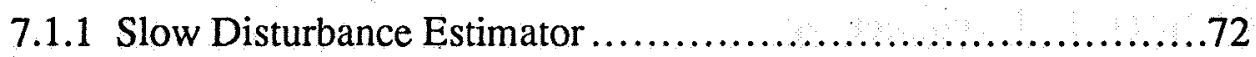

7.1.1.1 Rigid Body Case ..................................72

7.1.1.2 Flexible Body Case ...................................73

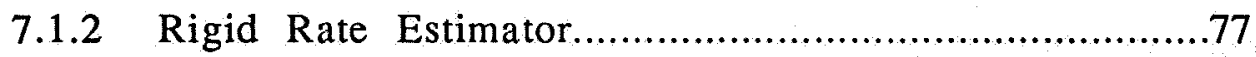

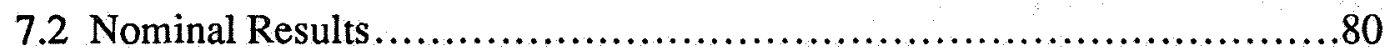

7.2.1 Orbiter Alone ................................................ 81

7.2.1.1 Maneuver Results .....................................83

7.2.1.2 Inertial Attitude Hold Results .........................91

7.2.2 Orbiter with GRO, Modeled as Rigid ........................98

7.2.2.1 Maneuver Results ....................................100

7.2.2.2 Inertial Attitude Hold Results ..........................105

7.2.3 Orbiter with GRO, Modeled as Flexible ......................111

7.3 Off Nominal Results ................................................ 122

7.3.1 Orbiter Alone ............................................... 123

7.3.2 Orbiter with GRO, Modeled as Rigid ...........................129

7.3.3 Orbiter with GRO, Modeled as Flexible .........................135

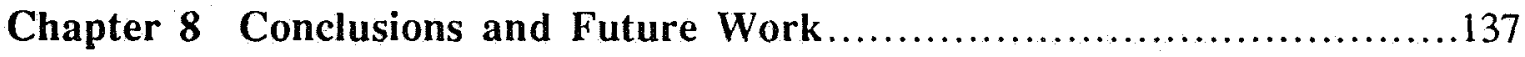

8.1 Recommendations for Future Work............................................140

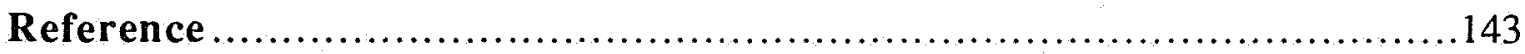




\section{List of Figures}

Figure 2.1. Small, Medium, and Big Membership Functions for $X, Y$, and $Z$.......21

Figure 2.2. Evaluation of Fuzzy Rules........................................22

Figure 2.3. Defuzzification of Rule Outputs ...................................23

Figure 4.1. Fuzzy Attitude Controller Block Diagram...................................30

Figure 4.2. The Jet Selection Parameters and Opposing Jets Selected for Small

Angle Error ..................................................... 34

Figure 4.3. Membership Functions for Pointing Error and Rate Error..................36

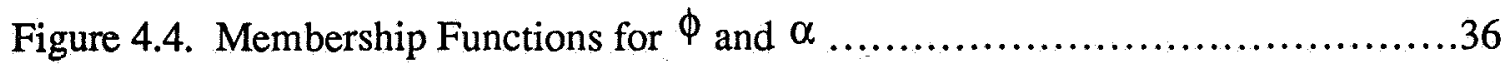

Figure 4.5. Allowable Error Envelope with Slow Disturbance Trajectory................38

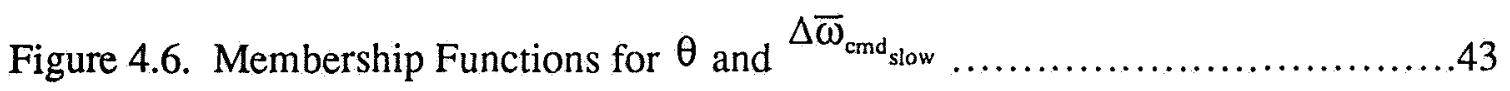

Figure 4.7. Membership Functions for Fuel Savings Package ......................44

Figure 4.8. Membership Functions for Anti-Chatter Rule Package.......................46

Figure 4.9. Membership Functions for Anti-Flex Rule Package $\ldots \ldots \ldots \ldots \ldots \ldots \ldots \ldots . . . .48$

Figure 6.1. OSRS Coordinate System .....................................60

Figure 6.2. Space Shuttle Jet Locations........................................61

Figure 6.3. Artist's Conception of Space Shuttle with Deployed GRO ................62

Figure 6.4. Space Shuttle Flight Digital Autopilot ...............................64

Figure 6.5. Simple Phase Plane ..................................................66

Figure 6.6. Phase Plane with Deadband and High Rate Limit Cycle ...................67

Figure 6.7. Phase Plane with Deadband Designed for Low Rate Limit Cycles ..........67

Figure 6.8. Space Shuttle Phase Plane ....................................68

Figure 7.1. Settling Times vs. Butterworth Filter Order for Various Input Frequencies................................................... 74

Figure 7.2. Peak Transient Amplitudes vs. Butterworth Filter Order ..................75

Figure 7.3. Frequency Response of 5th Order Butterworth Filter ......................76

Figure 7.4. Slow Disturbance Acceleration Time Histories with and without Flexible Disturbances and Higher Order Filtering ....................77

Figure 7.5. Frequency Response of Frequency Weighting Filter....................78

Figure 7.6. Total and Rigid Body Environment Rates ...............................79

Figure 7.7. Actual and Estimated Rigid Rates with and without Frequency Weighting......................................................... 79

Figure 7.8. Maneuver Results for Vernier Jets ...................................85

Figure 7.9. Results Averaged over all Maneuvers for Vernier Jets ....................86

Figure 7.10. Average Completion Time of Maneuvers for Vernier Jets..................87 
Figure 7.11. Maneuver Results for Alt Mode ......................................88

Figure 7.12. Results Averaged over all Maneuvers for Alt Mode............................89

Figure 7.13. Average Completion Time of Maneuvers for Alt Mode.......................89

Figure 7.14. Pitch Rate and Fuel Usage for a Maneuver in Alt Mode....................90

Figure 7.15. Inertial Attitude Hold Results for Vernier Jets .........................92

Figure 7.16. Average Inertial Attitude Hold Results for Vernier Jets.........................93

Figure 7.17. Average Eigenangle and Limit Cycle Time for Inertial Attitude Hold using Vernier Jets ................................................93

Figure 7.18. FCS Body Rates for a Segment of Hold B using Verniers...................94

Figure 7.19. Fuzzy Attitude Controller Body Rates for a Segment of Hold B using Verniers .........................................................94

Figure 7.20. Inertial Attitude Hold Results for Alt Mode ..........................96

Figure 7.21. Average Inertial Attitude Hold Results for Alt Mode.........................97

Figure 7.22. Average Eigenangle and Limit Cycle Time for Inertial Attitude Hold using Alt Mode................................................97

Figure 7.23. Maneuver Results for Rigid Orbiter-GRO with Verniers..................99

Figure 7.24. Average Maneuver Results for Rigid Orbiter-GRO with Verniers .........100

Figure 7.25. Average Maneuver Completion Time for Rigid Orbiter-GRO with Verniers ............................................................... 100

Figure 7.26. Body Rates and Eigenangle for Maneuver D with Verniers for FCS and Fuzzy with Baseline, Jet Limiting, and Anti-Chatter Rules.

Figure 7.27. Fuel Use and Jet Switches for Maneuver D with Verniers for FCS and Fuzzy with Baseline, Jet Limiting, and Anti-Chatter Rules.............102

Figure 7.28. Maneuver Results for Rigid Orbiter-GRO using Alt Mode...................103

Figure 7.29. Average Maneuver Results for Rigid Orbiter-GRO with Verniers .........104

Figure 7.30. Average Maneuver Completion Time for Rigid Orbiter-GRO using Alt Mode .............................................................. 104

Figure 7.31. Inertial Attitude Hold Results for Rigid Orbiter-GRO with Verniers.........106

Figure 7.32. Average Inertial Attitude Hold Results for Rigid Orbiter-GRO with Verniers .................................................... 107

Figure 7.33. Average Eigenangle and Limit Cycle Time for Inertial Attitude Hold with Rigid Orbiter-GRO and Verniers........................................107

Figure 7.34. Inertial Attitude Hold Results for Rigid Orbiter-GRO with Alt Mode.......108

Figure 7.35. Average Inertial Attitude Hold Results for Rigid Orbiter-GRO with Alt Mode

Figure 7.36. Average Eigenangle and Limit Cycle Time for Inertial Attitude Hold with Rigid Orbiter-GRO and Alt Mode........................................109

Figure 7.37. FCS Body Rates for a Segment of Hold C, Alt Mode....................110

Figure 7.38. Fuzzy Attitude Controller Body Rates for a Segment of Hold C, Alt Mode ............................................................110

Figure 7.39. 2-Norm of Flexible Mode Rates(deg/s) for Verniers..........................114

Figure 7.40. Averaged Results for Verniers ......................................115 
Figure 7.41. Body Rates and Fuel Usage for Verniers, Maneuver D, FCS 'Origl'......117

Figure 7.42. Body Rates and Fuel Usage for Verniers, Maneuver D, Fuzzy

Attitude Controller 'msfc'..............................................117

Figure 7.43. 2-Norm of Flexible Mode Rates(deg/s) for Alt Mode........................119

Figure 7.44. Averaged Results for Alt Mode........................................120

Figure 7.45. Body Rates and Fuel Usage for Alt Mode, Maneuver D, FCS

'Orig1' .........................................................121

Figure 7.46. Body Rates and Fuel Usage for Alt Mode, Maneuver D, Fuzzy Attitude Controller ' $m s$ '.

Figure 7.47. Average Limit Cycle Times for Verniers and Alt Mode........................122

Figure 7.48. FCS Body Rates and Eigenangle for Maneuver A using Verniers .........124

Figure 7.49. Fuzzy Attitude Controller Body Rates and Eigenangle for Maneuver A using Verniers ........................................................ 124

Figure 7.50. Comparison of Fuel Consumption and Jet Switches for Maneuver A using Verniers................................................ 125

Figure 7.51. FCS Body Rates and Eigenangle for Maneuver $\mathrm{C}$ using Alt Mode ........127

Figure 7.52. Fuzzy Attitude Controller Body Rates and Eigenangle for Maneuver C using Alt Mode ..................................................... 127

Figure 7.53. Comparison of Fuel Consumption and Jet Switches for Maneuver C using Alt Mode ...................................................... 128

Figure 7.54. FCS Body Rates and Eigen Angle for Maneuver A using Verniers.........130

Figure 7.55. Fuzzy Attitude Controller Body Rates and Eigen Angle for Maneuver

A using Verniers ....................................................130

Figure 7.56. Comparison of Fuel Consumption and Jet Switches for Maneuver A using Verniers ................................................ 131

Figure 7.57. FCS Body Rates and Eigenangle for Maneuver C using Alt Mode ........133

Figure 7.58. Fuzzy Attitude Controller Body Rates and Eigenangle for Maneuver C using Alt Mode.

Figure 7.59. Comparison of Fuel and Jet Switches for Maneuver C using Alt Mode 



\section{List of Tables}

Table 6.1. Jet Performance Specifications ........................................61

Table 6.2. Magnitude of Disturbances Acting on Space Shuttle...........................61

Table 6.3. Modal Frequencies of Combined Space Shuttle-GRO System ...............63

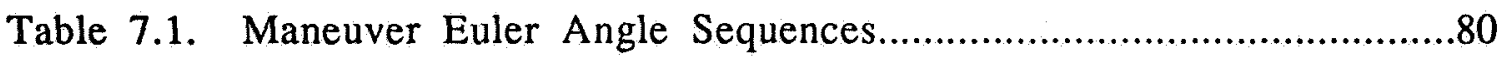

Table 7.2. Parameters for Space Shuttle Flight Control System ......................80

Table 7.3. Parameters for Fuzzy Attitude Controller .................................81

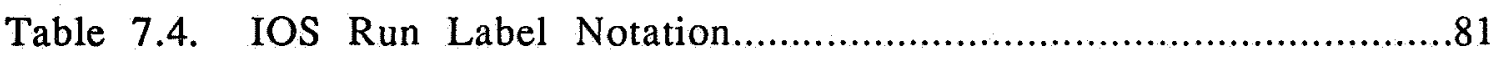

Table 7.5. Configurable Parameters for Fuzzy Attitude Controller, Orbiter Alone

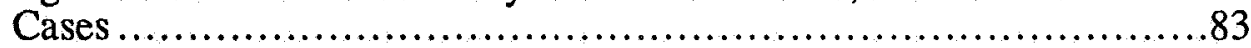

Table 7.6. Configurable Parameters for Fuzzy Attitude Controller Orbiter with GRO, Modeled as Rigid, Cases............................................98

Table 7.7. Configurable Parameters for Fuzzy Attitude Controller Orbiter with GRO, Modeled as Flexible, Cases............................................113

Table 7.8. Comparison of Nominal to Off Nominal Results for the FCS and Fuzzy Attitude Controllers using Verniers....................................126

Table 7.9. Comparison of Nominal to Off Nominal Results for the FCS and Fuzzy Attitude Controllers using Alt Mode ....................................129

Table 7.10. Comparison of Nominal to Off Nominal results with Rigid-GRO for the FCS and Fuzzy Attitude Controllers using Verniers ..................132

Table 7.11. Comparison of Nominal to Off Nominal Results with Rigid-GRO for the FCS and Fuzzy Attitude Controllers using Alt Mode

Table 7.12. Flex 2-Norms with and without Modeling Error for Verniers, Maneuver B

Table 7.13. Flex 2-Norms with and without Modeling Error for Alt Mode, Maneuver

C. 



\section{Chapter 1}

\section{Introduction}

Recent developments in the theory of fuzzy sets, originally developed by Zadeh[1], and its application through rules or "logic" suggests its use for controlling spacecraft attitude with on/off thrusters. Traditional attitude control system designs often use switching curves based on loaded constants and vehicle parameters, which may be approximations to the actual system values. Decisions are made whether to fire thrusters based on the relative value of the error in a desired state of the system with respect to these switching curves. These "phase plane" type controllers are based on the more traditional "crisp" set theory, where an object (e.g., attitude error) is either in or out of a set, but can have no partial membership.

Fuzzy set theory allows partial set membership. For example, the set of big attitude error is a fuzzy set which may overlap with the set of small attitude error. It is possible, depending on perspective, to be $75 \%$ within the big set and $25 \%$ within the small set. The intent being to represent the perspective or the "state" of the observer (i.e., there is a different interpretation of big and small attitude error depending on the classification of the rate error). A crisp set membership, might classify all attitude errors greater than 1 deg as big. This not only implies that an attitude error of $0.999 \mathrm{deg}$. is (100\%) small, but also removes the interdependence with the rate error, which also may have partial set membership. 
For the attitude control function of spacecraft, the objectives are to maintain a specified attitude within certain flight control error margins in the presence of disturbances and unmodeled dynamics. It is desired to do so with reasonable fuel consumption, limited jet firings, and without exciting flexible appendages or providing significant translation that may change the desired orbit. In any control implementation of these objectives, the objective must be defined as a function of the associated vehicle state. The objectives are interdependent and may be conflicting. Controllers that use "crisp" boundaries to discern small or big system states evaluate only specific objectives defined by the switching curve. This type of controller can experience thruster chattering by remaining too long in the vicinity of a switch curve since the boundary between big and small is "crisp". Crisp controllers are also more cumbersome to implement when conflicting objectives exist. For example, it may be beneficial to avoid a thruster firing, which may excite the bending dynamics of an appendage, even if the attitude error is big, but the rate error is small. Delaying the firing for a short period of time may allow the firing to occur at a better time in terms of flex excitation without causing the attitude error to become significantly larger. Implementing crisp boundaries for this kind of scenario would be cumbersome.

A fuzzy logic based control strategy is adept at dealing with the nonlinear equations of motion associated with spacecraft rotational dynamics and on/off actuators simply because it does not rely on analytical models, but instead upon rules developed according to the expertise of the design engineer. The linguistic nature of the rules simplifies the design process as well as the controller. Fuzzy logic also handles conflicting priorities since rules are easily written to penalize undesirable attributes while promoting desirable ones. For example, a sequence of rules can make the tradeoffs in the process of jet selection between maximizing the acceleration produced, minimizing the angle between the commanded direction and the actual direction, minimizing fuel consumption, and damping a flexible mode. 
Several papers from the recent literature discuss development of fuzzy based attitude controllers for spacecraft. Lea and Jani[2] developed a fuzzy based attitude controller designed to hold inertial attitude and perform constant rate maneuvers, but does not deal with disturbances or flexible body dynamics. Berenji[3] describes an attitude controller design, which attempts to mimic Space Shuttle phase plane controller objectives and compares results to a version of the Space Shuttle attitude control system. This thesis presents a generic three degree of freedom attitude controller which addresses the objectives listed above with the assumptions that spacecraft inertia is known, attitude and rate are measurable, actuators are stationary on/off thrusters, and models of the flexible dynamics are available. The fuzzy designs are to be easily adaptable to a variety of vehicles with differing missions and must accommodate different mass properties, assorted disturbances, and flexible modes. The approach taken is to develop a controller which employs fuzzy logic encompassed in packages of rules dedicated to addressing different objectives. These rule packages can be inserted or removed depending on the requirements of the spacecraft.

The thesis is organized as follows. Chapter 2 provides a brief outline of fuzzy set theory and logic. Chapter 3 describes the equations of motion for the rotational dynamics of a spacecraft, including disturbances. Chapter 4 discusses the approach and design of the fuzzy rule packages. Chapter 5 discusses possible design methodologies for estimating signals required by the fuzzy attitude controller. Chapter 6 provides a brief overview of the space shuttle orbiter and its flight control system. Chapter 7 presents performance results from space shuttle on-orbit operations using the space shuttle flight control system and the fuzzy attitude controller. Chapter 8 summarizes the work and discusses potential future work. 



\section{Chapter 2}

\section{Fuzzy Set Theory and Logic}

Fuzzy set theory, developed by Zadeh[1], is an extension of traditional or crisp set theory. Crisp set theory states that an item either is or is not a member of a set. The set boundaries are precise, leading to abrupt changes from membership to non-membership. Fuzzy sets are those ill-specified and not distinct collections of objects with "non-crisp" boundaries in which transition from membership to nonmembership is gradual, Maiers[4], such as the set of hot temperatures or bald men. Membership in a fuzzy set is defined by a membership function which ranges in value from zero to one, zero indicating nonmembership and one indicating full membership. The membership function is usually denoted by $\mu$. Figure 2.1 shows the membership functions for the fuzzy sets small, medium, and big, defined for the quantities $X, Y$, and $Z$.
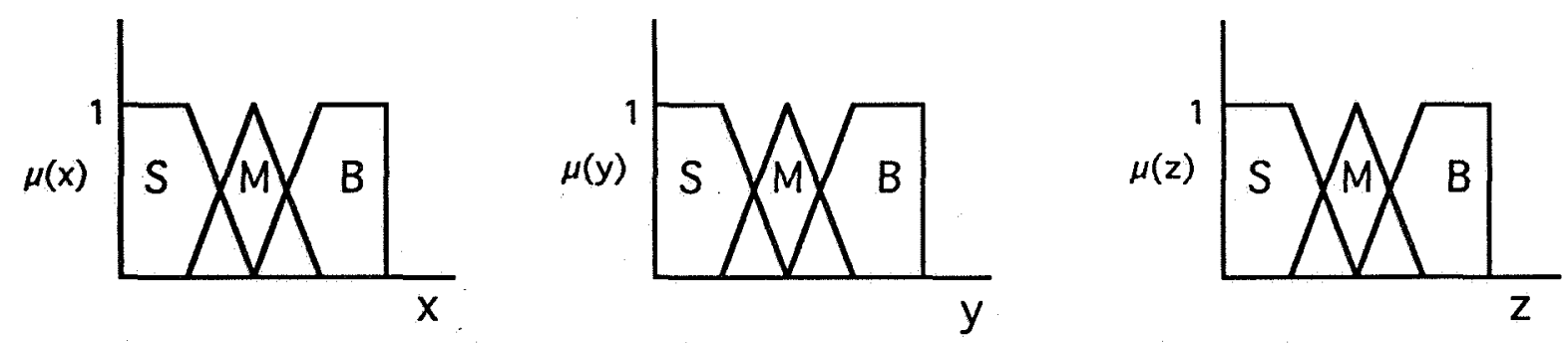

Figure 2.1. Small, Medium, and Big Membership Functions for $\mathrm{X}, \mathrm{Y}$, and $\mathrm{Z}$

Fuzzy logic is the application of fuzzy sets to rules. The following are typical of how fuzzy rules are constructed: 
If $\mathrm{x}$ is big and $\mathrm{y}$ is medium then $\mathrm{z}$ is big

If $\mathrm{x}$ is medium or $\mathrm{y}$ is big then $\mathrm{z}$ is medium

To evaluate these rules (Figure 2.2) the first step is to "fuzzify" the inputs $x$ and $y$. For the first rule, $x$ is fuzzified by assessing its membership in the fuzzy set big. Similarly, $y^{\prime}$ s membership in the fuzzy set medium is assessed. Rules whose predicates have multiple inputs, as in this case, use 'and' or 'or' operations to combine the membership value of each respective input into one membership value. In the case of 'and' operations the minimum membership value is used, for 'or' operations the maximum is used. The resultant membership value for the rule's predicate is used as the truth value for that rule. The truth value of the first rule would be x's membership in big since this is less than y's membership in medium. The output of the rule is set to be the area of the fuzzy set big which lies below the truth value. The same process is followed to evaluate the second rule.

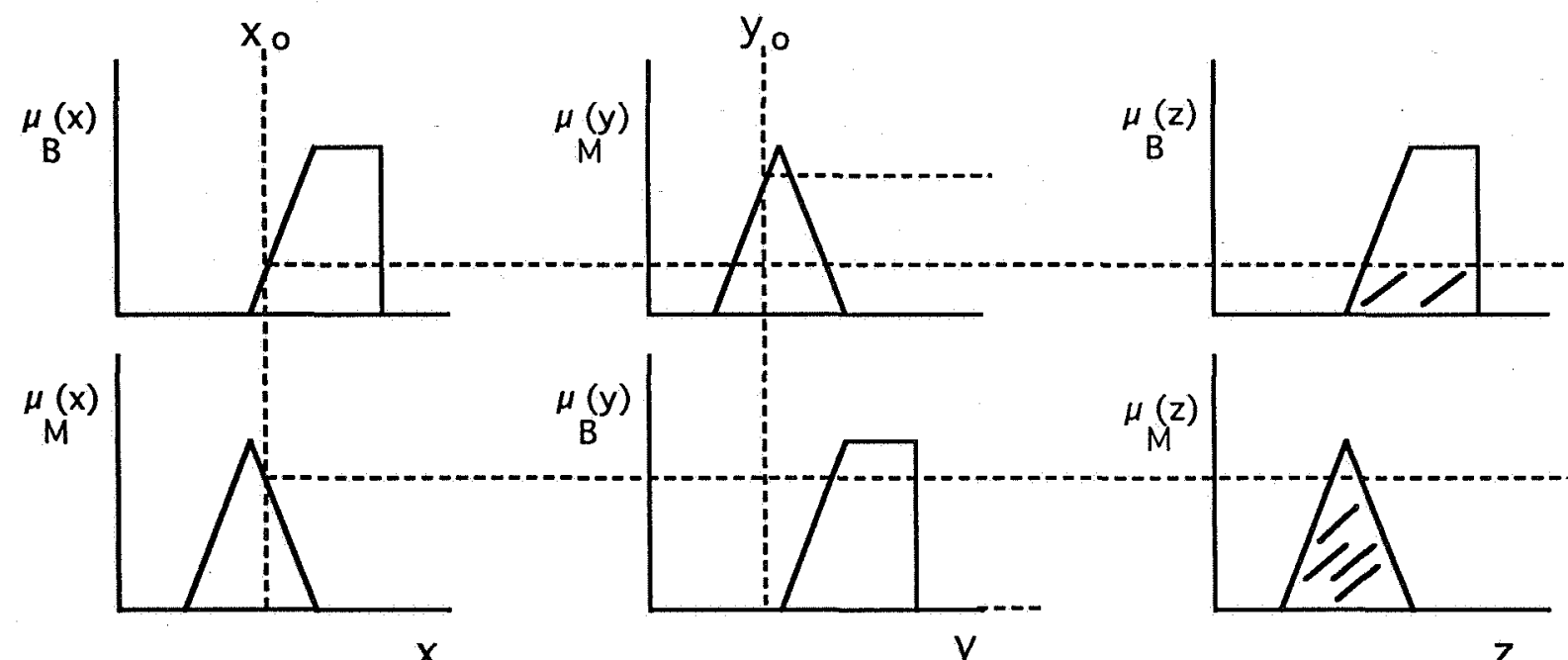

Figure 2.2. Evaluation of Fuzzy Rules

The last step is to "defuzzify" the combination of the rule outputs to get a crisp value for $z$. Many strategies exist for the defuzzification process. A common one is to take the union of the output areas from each rule and calculate the centroid of the resultant area. The location of the centroid is then used as the crisp output. 


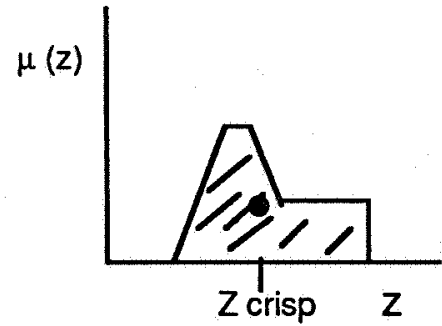

Figure 2.3. Defuzzification of Rule Outputs

A less computationally intense approach for finding a crisp output is to use weighted averages, Stephanides[5]. When employing this technique, each rule has an output value associated with it rather than a fuzzy set. The resulting crisp output value from a series of rules is the average of the rule outputs, weighted according to each rule's truth value. In the case of the previous rules, for example, Big may have the value 5 and Medium the value 3. The crisp output is found with Eq. (2.1) where $\mu_{\mathrm{B}}(\mathrm{x})$ and $\mu_{\mathrm{M}}(\mathrm{x})$, $\mathrm{x}$ 's membership in the fuzzy sets big and medium, are the truth values for the two rules.

$$
\mathrm{Z} \text { crisp }=\frac{\mu_{\mathrm{B}}(\mathrm{x}) * \operatorname{Big}+\mu_{\mathrm{M}}(\mathrm{x}) * \text { Medium }}{\mu_{\mathrm{B}}(\mathrm{x})+\mu_{\mathrm{M}}(\mathrm{x})}
$$

There are several issues the control engineer must consider when designing a fuzzy controller. The first consideration is how many different areas of operation are there within the state space of the system to be controlled. This information is used to partition the state space with fuzzy sets such as small, medium, and big. Rules can then be constructed based upon these fuzzy sets whose outputs effect the desired behavior. Since the number of rules required is related to the resolution of the state space partitioning, it may be more efficient to use fewer partitions. This will generally be successful in cases where the desired output from the fuzzy controller operating in a given regime of the state space is somewhere between the controller outputs from the areas on either side. For example, it may be possible to replace the fuzzy sets small, medium, and big with small and big. 
Another issue is the rate at which transition from one area of the state space to another occurs. This can be controlled by adjusting the shape of the fuzzy membership functions which divide the different areas of the state space. In cases where fuzzy sets are eliminated, the adjacent fuzzy sets are usually extended to overlap across that region.

Once the engineer has developed rules according to his expert knowledge of the way the system should behave, the controller in the loop with the system should be tested for stability and robustness. Chen and Tsao[6] and Chen[7] present useful approaches for performing this analysis. 


\section{Chapter 3}

\section{Dynamics}

The basic rotational equations of motion for a spacecraft are, Kaplan[8]:

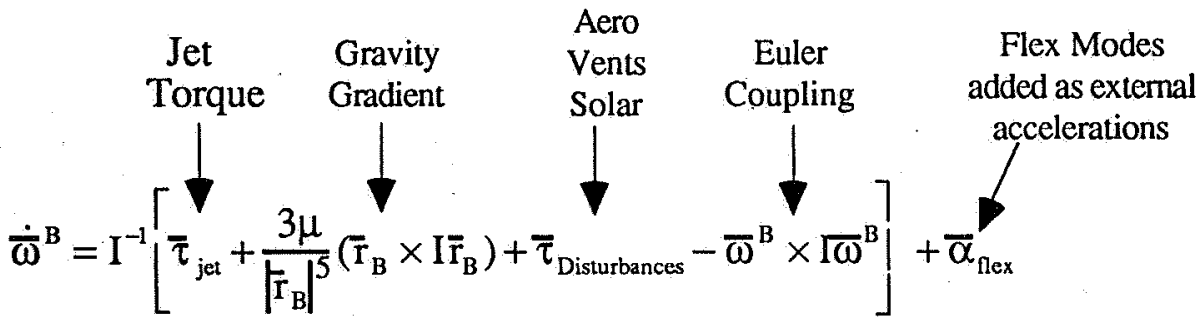

$$
\begin{aligned}
& \dot{\mathrm{q}}_{\mathrm{I}}^{\mathrm{B}}=\frac{1}{2} \Omega \mathrm{q}_{\mathrm{I}}^{\mathrm{B}} ; \quad \Omega=\left[\begin{array}{cc}
0 & \bar{\omega}_{\mathrm{B}}^{\mathrm{T}} \\
-\bar{\omega}_{\mathrm{B}} & -\bar{\omega}_{\mathrm{B}} \times
\end{array}\right] ; \quad \bar{\omega}_{\mathrm{B}} \times=\left[\begin{array}{ccc}
0 & -\omega_{3} & \omega_{2} \\
\omega_{3} & 0 & -\omega_{1} \\
-\omega_{2} & \omega_{1} & 0
\end{array}\right] \\
& \mathrm{q}_{\mathrm{I}}^{\mathrm{B}}=\left[\begin{array}{c}
\cos \frac{\theta}{2} \\
-\overline{\mathrm{v}} \sin \frac{\theta}{2}
\end{array}\right] ; \quad \begin{array}{c}
\theta \text { is the eigenangle } \\
\overline{\mathrm{v}} \text { is the eigenaxis }
\end{array}
\end{aligned}
$$

Eq. (3.1) presents Euler's moment equation. It relates the angular acceleration of the spacecraft in body frame to the external moments applied to it. Included in the equation are the jet torque; torque due to gravity gradient where $\overline{\mathrm{r}}_{\mathrm{B}}$ is the position of the spacecraft in 
body axes with respect to the earth's center and $\mu$ is the gravitational constant; and torque due to other disturbances such as aero loading, solar pressure, and venting. It also includes torque due to Euler or gyroscopic coupling and angular acceleration due to flexible modes. Integrating Eq. (3.1) gives body rates which are used in Eq. (3.2) to propagate $\mathrm{q}_{\mathrm{I}}^{\mathrm{B}}$, the quaternion that relates the current spacecraft body attitude to an inertial reference frame. The elements of $\mathrm{q}_{\mathrm{I}}^{\mathrm{B}}$ are defined in Eq. (3.3). (For more information on quaternions see Carroll[9])

The effects of flexible modes are assumed to be included as an external acceleration on the spacecraft center of gravity (cg). In general $\bar{\alpha}_{\text {flex }}$ is a non-linear function of $\mathrm{q}_{1}^{\mathrm{B}}, \dot{\mathrm{q}}_{1}^{\mathrm{B}}, \bar{\tau}_{\text {jet }}$, and the flex states. However, if the actuation capability of the spacecraft is large compared to the disturbances, the flexible dynamics can be simplified by neglecting the effect of these disturbances on the flexible dynamics. A linear model of the flexible dynamics which takes as inputs the jets fired and outputs an acceleration about the spacecraft cg can be developed, $\mathrm{Eq}(3.4)$.

$$
\begin{aligned}
& {\left[\begin{array}{c}
\overline{\mathrm{w}} \\
\overline{\mathrm{w}}
\end{array}\right]=\left[\begin{array}{cc}
0 & \mathrm{I} \\
-\omega^{2} & -2 \zeta \omega
\end{array}\right]\left[\begin{array}{l}
\overline{\mathrm{w}} \\
\overline{\mathrm{w}}
\end{array}\right]+\left[\begin{array}{c}
0 \\
\mathrm{~B}_{\text {jels }}
\end{array}\right] \overline{\mathrm{u}} } \\
&\left.\bar{\alpha}_{\text {flex }}=\left[\begin{array}{ll}
0 & \alpha_{36}
\end{array}\right] \begin{array}{cc}
0 & \mathrm{I} \\
-\omega^{2} & -2 \zeta \omega
\end{array}\right]\left[\begin{array}{c}
\overline{\mathrm{w}} \\
\overline{\dot{\mathrm{w}}}
\end{array}\right]+\left[\begin{array}{ll}
0 & \alpha_{36}
\end{array}\right]\left[\begin{array}{c}
0 \\
\mathrm{~B}_{\text {jels }}
\end{array}\right] \overline{\mathrm{u}} \\
& \overline{\mathrm{u}}= \text { Jet on vector }(1=\text { on }) \\
& \overline{\mathrm{w}}= \text { flex mode amplitudes } \\
& \omega= \text { natural frequencies } \\
& \zeta= \text { damping ratio } \\
& \alpha_{36}= \text { transforms flex states to spacecraft rotational } \\
& \text { deg rees of freedom about the c.g. }
\end{aligned}
$$

The $-\omega^{2}$ and $-2 \zeta \omega$ terms in Eq (3.4) are diagonal matrices. Each diagonal element contains the frequency and damping information for another mode. 
The disturbance terms, noted in Eq. (3.1), can be separated into two classes, low frequency and high frequency. In cases where the spacecraft is performing an attitude hold with respect to inertial space the gravity gradient will be periodic with the orbit. Disturbances due to the atmosphere will also be periodic with the orbit because of orbital eccentricity and the difference in solar heating between the front and back sides of the earth. In most cases the solar pressure will be periodic with the orbit as well. These are classified as low frequency disturbances. The rest of the disturbance terms are classified as high frequency disturbances since their frequency content depends on thruster firing patterns and flexible body dynamics.

The fuzzy logic attitude controller will contain rule packages to deal separately with the low frequency and high frequency disturbances. This will allow flexibility in moving from one spacecraft to another where each term in Euler's moment equation is liable to carry different levels of importance. 



\section{Chapter 4}

\section{Fuzzy Attitude Controller}

A fuzzy attitude controller was developed for controlling a generic spacecraft's attitude without specific knowledge of the governing non-linear equations of motion. The fuzzy framework provides a simple means of extending the capabilities of the controller by allowing the insertion of more rules to address additional issues. This non-dependence upon specific dynamics and the inherent flexibility allow this attitude controller to be applicable to a wide class of spacecraft, as well as many different operations for a given spacecraft.

To facilitate the design process, there are some general assumptions that must be made about the spacecraft to be controlled. The mass properties of the spacecraft must be known. Accurate attitude and rate measurements must also be available. If the rate is estimated, it should not contain significant phase lag, especially at the frequency of any flexible modes that require damping. The actuators are assumed to be non-vectorable, on/off jets operated with a control system sampling interval, $\Delta t$. The position and direction of the jets along with the magnitude of their thrust should be known. If flexible modes are to be controlled, a linear model of the flexible dynamics should be available.

The fuzzy attitude controller will accommodate certain performance specifications typically 
required by the vehicle designers. These specifications are input to the fuzzy controller through what will be referred to as the set of configurable parameters. Two of the major configurable parameters are the maximum amount of pointing error or the pointing error constraint and the desired rate at which maneuvers should be conducted. Other configurable parameters include the maximum number of jets allowed to be fired simultaneously (Maxjets) and the maximum allowable error in rate (rate error constraint).

Typical spacecraft attitude controllers separate the task of attitude control into two stages. The first stage determines if, and in what direction, a firing should be made. Phase planes for each axis are often employed for this task, the axes of the phase planes being rate and attitude error for roll, pitch, and yaw. Each phase plane has a dead zone in the center with switching curves emanating from it. If the error for an axis is inside the deadzone, that axis does not command a firing. If a switch curve is crossed then that axis commands a firing in the appropriate direction. The composite firing direction is then passed to a jet selection logic which finds the best combination of jets to accomplish the requested rate change and commands them to be fired. One of the problems with this approach is that it assumes the axes can be dealt with individually. Unfortunately, this is not often the case. Depending on the mass properties, firings in one axis can cause significant rate changes in another.

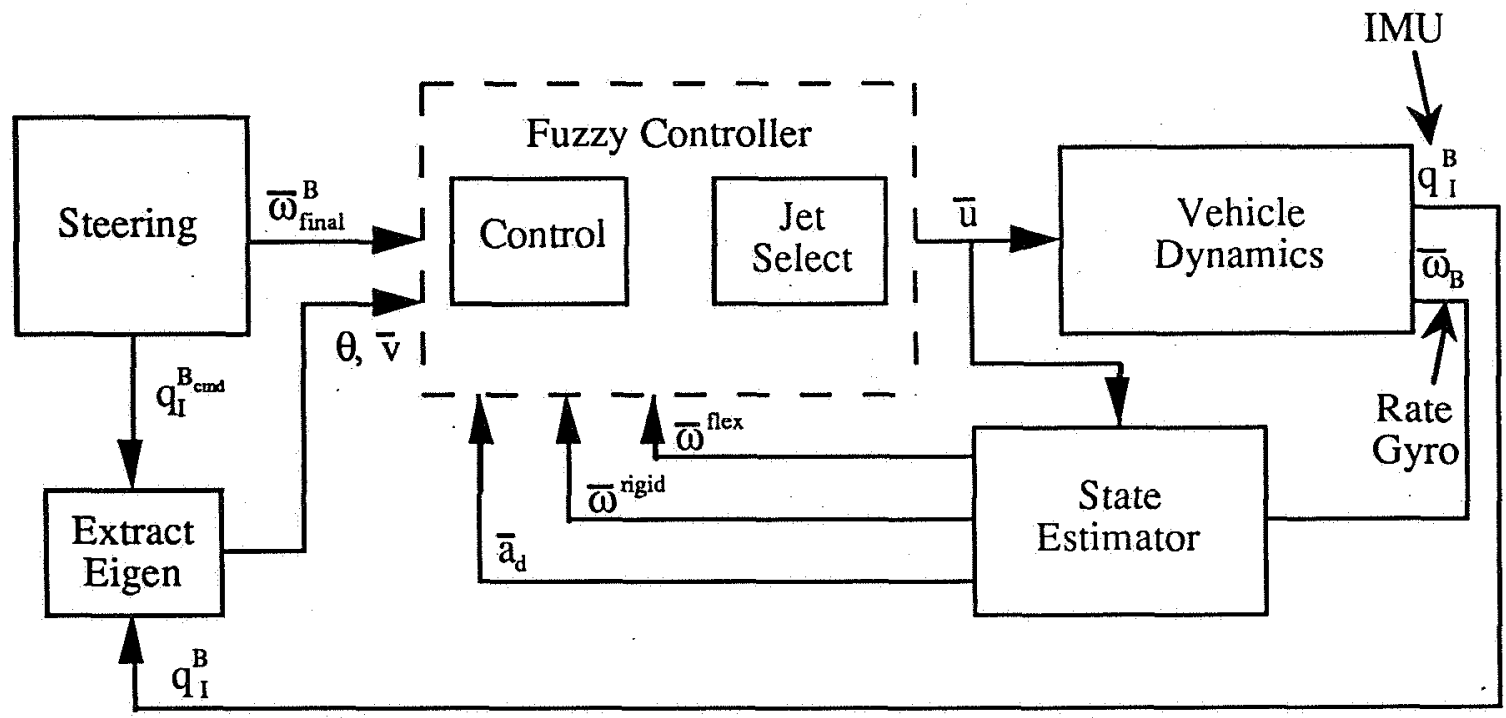

Figure 4.1. Fuzzy Attitude Controller Block Diagram 
The fuzzy attitude controller shown in Figure 4.1 replaces the spacecraft phase planes and jet selection modules with a one step, multi-axis process. This satisfies the important objective that the decision on whether to fire should depend on the quality of the available jet selection as well as the fact that the decision to fire in one axis should not be made independent of the decision in other axes. The fuzzy controller takes as input the estimated rigid and flex components of the body rate, $\bar{\omega}^{\text {rigid }}$ and $\bar{\omega}^{\text {flex }}$, an estimate of the disturbance acceleration acting on the spacecraft, $\bar{a}_{d}$, the eigenangle, $\theta$, and eigenaxis, $\bar{v}$, between the current attitude and the desired attitude as well as the body rates the spacecraft should have upon attaining the desired attitude, $\bar{\omega}_{\text {final }}^{\mathrm{B}}$. The eigenangle and eigenaxis are based upon Euler's Theorem: The most general displacement of a rigid body with one point fixed is equivalent to a single rotation about some axis through that point, Greenwood[10]. The eigenaxis is the axis about which rotation will move the body from the current attitude to the desired attitude. The eigenangle is the angle of rotation required about the axis. The fuzzy controller outputs the vector of jets to be fired, $\bar{u}$.

\subsection{Objectives}

There are several primary objectives for an attitude controller which must be addressed. The first is to maintain an attitude relative to some frame of reference (LVLH, Inertial, Solar Inertial), in the presence of disturbances, while using a reasonable amount of fuel. The capability must also exist to move from one attitude to another in an appropriate amount of time, where the final attitude may be fixed in inertial space or tracking a point such as the center of the earth or another orbital object. Further considerations, which affect jet lifetime, are minimizing the number of on/off cycles and the total on-time of individual jets.

These objectives imply the following requirements. First, efficient limit cycles should be set up to avoid jet chatter. This can occur when a trajectory is being followed closely or 
when some threshold requiring a jet firing is crossed repeatedly due to a disturbance acceleration. This is a problem common to controllers using crisp switch curves. Second, the rate at which the error is removed should be related to the magnitude of the error. This prevents large, fuel costly acceleration and deceleration burns for small errors. Third, hysteresis regions should exist to aid fuel conservation. Finally, jets should be selected such that their resultant is a small angle error between the desired and predicted directions of acceleration while acceleration along the desired direction is large.

\subsection{Baseline Rule Set}

The baseline rule set provides the most basic attitude control capability, determining which jets should be fired, if any, given the current and desired attitudes. No attempt is made to deal with disturbances in an intelligent manner. It meets the primary objectives of maintaining a specified attitude within specified error bounds, performing a timely maneuver, and to à certain degree, fuel conservation and prevention of jet chatter. It also allows for configuration of the maximum amount of error and the maneuver rate.

The fuzzy attitude controller works to drive the eigenangle, $\theta$, to zero. If $\theta$ is large enough, the rate at which it can be zeroed is limited by the selected maneuver rate. Since the assumed spacecraft uses on/off thrusters, the error can not be driven exactly to zero. As the residual body rates cause the spacecraft to drift from its desired attitude, appropriate jets must be selected for firing when the error reaches its maximum allowable value.

The following two preliminary rules control the rate at which the attitude controller will remove the attitude error. For smaller errors, a slower rate is commanded. As the error becomes big, the commanded rate approaches the configurable parameter, maneuver rate.

If $\theta$ is small then $\dot{\theta}_{\text {cmd }}$ equals zero.

If $\theta$ is big then $\dot{\theta}_{\text {cmd }}$ equals the maneuver rate. 
The output, $\dot{\theta}_{\text {cmd }}$ (commanded rate of change of the eigenangle), is subtracted from the actual rate of change of the eigenangle, Eq. (4.2), which is found by taking the dot product of the eigenaxis, $\bar{v}$, and the body angular rate, Eq. (4.1). Here the eigenaxis represents the rotation axis from the current inertially referenced body attitude to the commanded attitude as provided by the steering logic (see Figure 4.1).

$$
\begin{gathered}
\dot{\theta}=\bar{\nabla} \bullet \bar{\omega}^{\mathrm{B}} \\
\dot{\theta}_{\text {error }}=\dot{\theta}-\dot{\theta}_{\text {cmd }}
\end{gathered}
$$

This gives the rate error about the eigenaxis which is used in conjunction with the desired rate change in body axes found in Eq. (4.3) below, to determine which jets, if any, should be fired.

$$
\Delta \bar{\omega}_{\mathrm{cmd}}=\dot{\theta}_{\mathrm{cmd}} \bar{v}-\bar{\omega}^{\mathrm{B}}+\bar{\omega}_{\mathrm{final}}^{\mathrm{B}}
$$

The challenge to performing a jet selection on the assumed spacecraft is that the thrusters are fixed in direction, location, and thrust, while the inertia may be highly variable from spacecraft to spacecraft or even within a single spacecraft over the profile of its mission. Several jet selection methods have been used in the past on a variety of spacecraft. These can generally be divided into two categories, "optimal" jet selections and "dot product" jet selections. The former method often requires significant storage capacity and cpu loading, and for this reason, we have opted for the latter. Traditional dot product schemes output a control each sample time based on the dot product of a jet's (or group of jets') resultant acceleration relative to the commanded acceleration. This is accomplished by precomputing angular rate increments that represent the angular rate change provided by a particular 
thruster over the sample time, $\Delta t$. If $\Delta t$ is small, only the direction of acceleration is important. The final rate error after the burn will be less than or equal to the rate change from one $\Delta t$ impulse. The tradeoff to be made here is between maximizing the amount of acceleration in the desired direction, $\alpha$, and minimizing the angle between the desired and predicted directions, $\phi$, using the minimum number of jets (Figure 4.2). If a strategy which only considers angle minimization is used, a selection of opposing jets may be made, which wastes fuel and does not provide much net acceleration. Furthermore, looking for combinations of jets which maximize the component of acceleration along the desired direction can introduce large components perpendicular to the desired direction which must be corrected later.
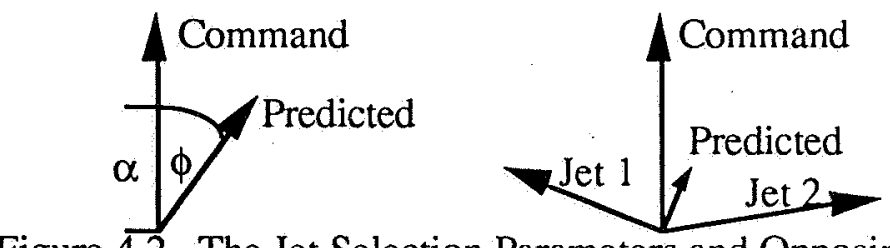

Figure 4.2. The Jet Selection Parameters and Opposing Jets Selected for Small Angle Error

The baseline fuzzy jet selection module contains six simple rules which are used to rank all possible combinations of thrusters with no more than a selectable number of simultaneously firing jets (Maxjets). This includes the case where no jets fire for which $\alpha$ and $\phi$ are taken to be zero. The output of each rule is either good, bad, or very bad which are given the values 1,0 , and -1 , respectively. The rule output, very bad, was used to create a greater differential between jet selections with small and big $\alpha$ while $\dot{\theta}_{\text {error }}$ is big. Since the jet selection firing no jets will have $\alpha$ equal to zero, it will look worse relative to other possible jet selections, helping to ensure that even when only poor jet selections are available to reduce $\dot{\theta}_{\text {error }}$, they will be preferred over the no jet selection case. 
If $\dot{\theta}_{\text {error }}$ is small and $\alpha$ is small then jet selection is good

If $\dot{\theta}_{\text {error }}$ is small and $\alpha$ is big then jet selection is bad

If $\dot{\theta}_{\text {error }}$ is big and $\alpha$ is small then jet selection is very bad

If $\dot{\theta}_{\text {error }}$ is big and $\alpha$ is big then jet selection is good

If $\phi$ is big then jet selection is bad

If $\phi$ is small then jet selection is good.

The average of the rule outputs weighted according to each rule's truth value is calculated for each jet selection possibility. The jet selection with the greatest resulting crisp output is then fired or in the jet selection case with no jets on, not fired. When $\dot{\theta}_{\text {error }}$ is large these rules favor a jet selection which will efficiently reduce the error. Jet selections which contain opposing jets (small angle and small acceleration) are penalized, and jet selections which have large direction error, even if the dot product with the desired direction is large, are also penalized. In the case when $\dot{\theta}_{\text {error }}$ is small the rules favor a jet selection which has no acceleration (i.e. no jets fire). This provides the hysteretic effect required to prevent continual jet firings since the error can never be completely zeroed out.

The relevant membership functions for the baseline controller are shown in Figures 4.3 and 4.4. The first set of membership functions in Figure 4.3 were sized according to the lead angle, the change in attitude which will occur while a rate change equal to the maneuver rate is conducted. This is approximated by

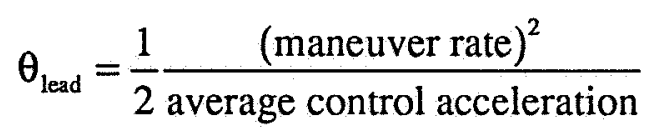

The second set of membership functions represents the amount of error in rate that is tolerated. They are sized according to the configurable parameter, rate error constraint. 

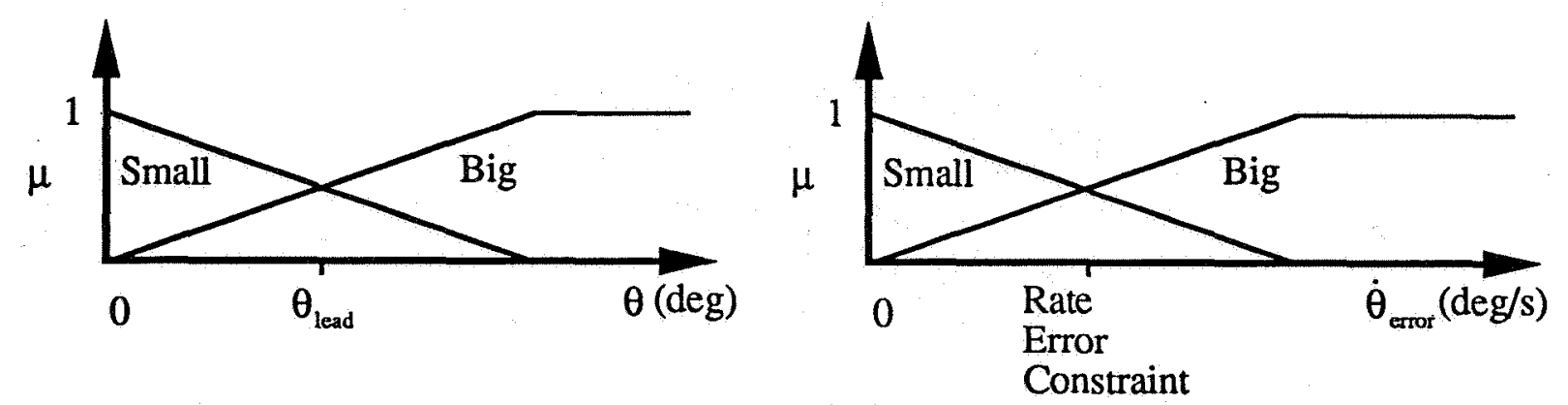

Figure 4.3. Membership Functions for Pointing Error and Rate Error

Figure 4.4 defines the membership functions for $\phi$ and $\alpha$. Small and big $\phi$ were set to cover the entire range of possibilities. Small and big $\alpha$ were adjusted according to the configurable parameter average control acceleration which represents the average rate change the spacecraft can achieve in $\Delta t$ about any given eigenaxis.
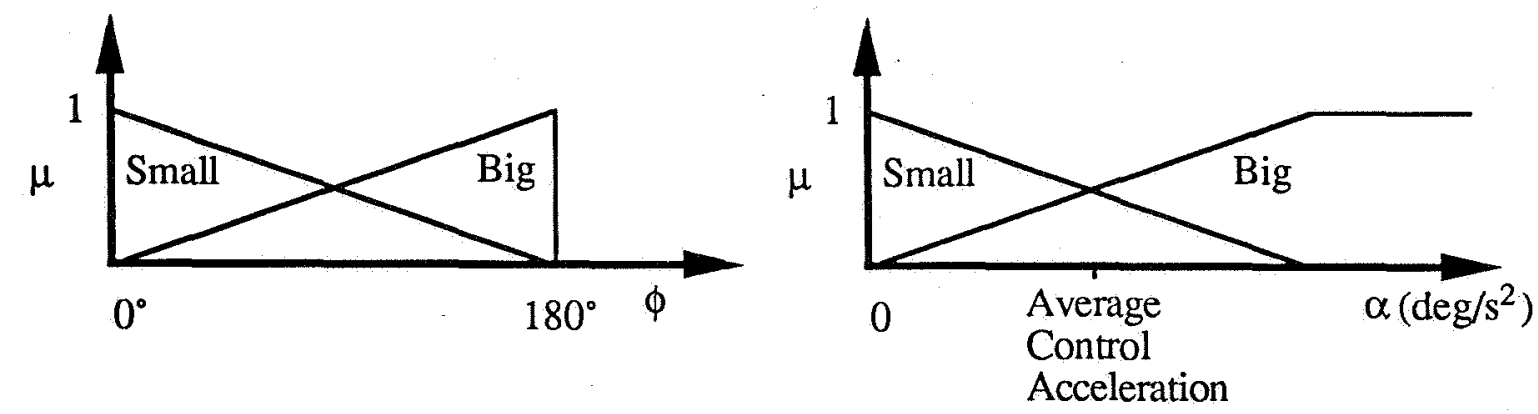

Figure 4.4. Membership Functions for $\phi$ and $\alpha$

The shape of the membership functions which are sized automatically according to configurable parameters of the fuzzy attitude controller, were chosen for computational simplicity. More complicated functions might be employed if it was desired to vary the rate of transition from one fuzzy set to another, but it is unclear what benefit this would provide within the framework of these rules. The membership functions overlap across the entire region the values of the given state variables are expected to lie within, always allowing the fuzzy rules to differentiate changes in their quantities.

This fuzzy attitude controller does not require the spacecraft attitude to follow a certain 
trajectory while reducing the eigenangle, it only requires that the eigenangle decrease with a large enough rate. Since a specific trajectory is not required to be tracked during attitude maneuvers, jet on/off cycles will be reduced in many cases over schemes which track a trajectory throughout an attitude maneuver. The rules given above for evaluating jet combinations provide the means for trading off (or interpolating between) the maintenance of good acceleration and the prevention of large directional errors.

\subsection{Supplemental Rule Packages}

The rule packages described in the following sections are designed to meet additional objectives beyond the baseline rule set. To activate them, they are simply added to the list of baseline rules. The relative weighting for each rule package, $w_{i}$, can be adjusted for spacecraft which place different relative importance among the phenomena the rule packages address.

$$
\text { total output }=\frac{\sum_{i} w_{i} * \text { output }_{i}}{\sum_{i} w_{i}}
$$

The final output, which can be used to compare the desirability of one jet selection versus another, is the weighted average of all the rule package outputs, output.

\subsection{Slow Disturbances Rule Package}

The slow disturbances are those that vary according to the position of the satellite within its orbit and can be treated as constant over the course of a limit cycle. These disturbances can keep the spacecraft attitude error on one side of the error envelope, leading to jet chattering and an excessive fuel consumption rate. (The error envelope contains all possible attitudes which do not exceed the maximum allowable pointing error). The solution is to allow the spacecraft attitude error to excurse to the opposite side of the allowable error envelope and 
let the disturbances pull it back. This results in longer, more fuel efficient limit cycles and eliminates jet chattering.

This suggests a trajectory which is aligned with the disturbance acceleration and travels from one side of the error envelope to the other before the rate is reversed by the disturbance acceleration. Due to the discrete nature of the on/off thrusters available to perform the required rate change, attempting to excurse to the opposite side of the error envelope is not advisable as the actual rate achieved may be too great producing a trajectory which will exceed the pointing error constraint and subsequently cause firings to eliminate the extra rate. A more conservative approach is to choose a point short of the opposite side of the error envelope as the apex of the trajectory.

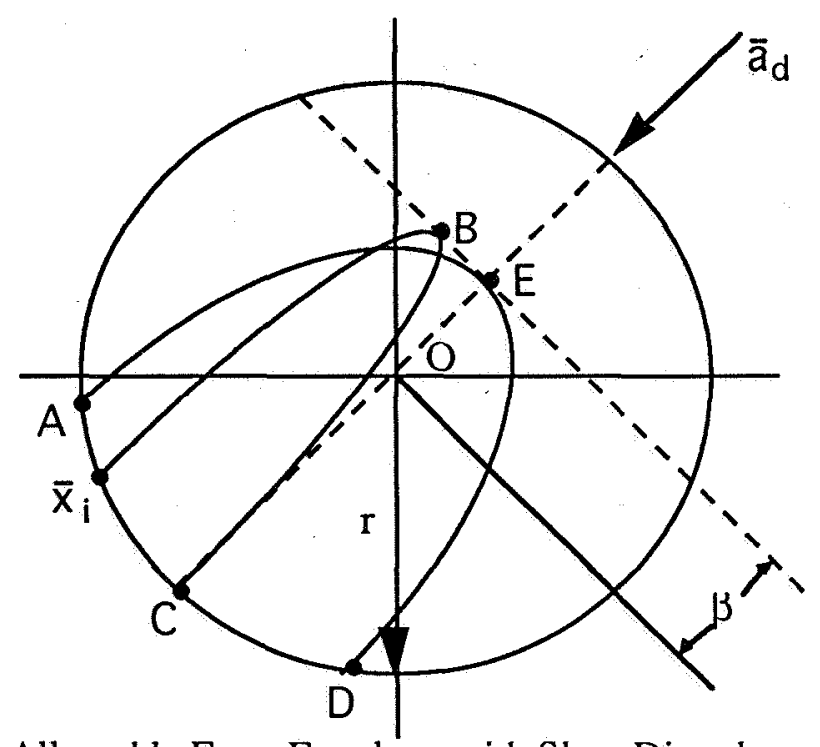

Figure 4.5. Allowable Errọ Envelope with Slow Disturbance Trajectory

The trajectory solution is based upon, Bergmann[11]. Consider the situation in Figure 4.6, where $\bar{x}_{i}$ represents the current attitude and $\bar{a}_{d}$ the disturbance acceleration. The goal is to find $\overline{\dot{x}}_{i}$ such that point $B$ is achieved followed by a coast to point $C$. The perpendicular displacement of point $B$ relative to $\bar{a}_{d}$ is set such that the trajectory does not overshoot and arrive at point $D$. $\beta$ is a metric of the trajectory as shown in Figure 4.5. After the initial 
limit cycle this trajectory will approach the desired trajectory described above, where all rate changes acts against the disturbance.

To find the required rate, $\overline{\mathbf{x}}_{i}$, begin with the expression for the rate at point $B$ where $t_{1}$ is the time required to reach point $B$.

$$
\overline{\dot{x}}_{B}=\overline{\dot{x}}_{i}+\bar{a}_{d} t_{1}
$$

Take the component of $\overline{\dot{x}}_{B}$ parallel to the disturbance by dotting with $\overline{\mathrm{i}}_{d}$, a unit vector along the disturbance,

$$
\overline{\dot{x}}_{B} \cdot \bar{i}_{d}=\left(\overline{\dot{x}}_{i}+\bar{a}_{d} t_{1}\right) \cdot \bar{i}_{d}
$$

and note that at time $\mathrm{t}_{1}$ this component is 0 giving

$$
\overline{\dot{x}}_{i} \cdot \overline{\mathrm{i}}_{d}=-\overline{\mathrm{a}}_{\mathrm{d}} \mathrm{t}_{1} \cdot \overline{\mathrm{i}}_{\mathrm{d}}
$$

Solving for $t_{1}$ gives

$$
t_{1}=-\frac{\overline{\dot{x}}_{i} \cdot \bar{i}_{d}}{\bar{a}_{d} \cdot \bar{i}_{d}}=-\frac{\overline{\dot{x}}_{i} \cdot \bar{i}_{d}}{\left|\bar{a}_{d}\right|}
$$

The attitude at point $B$ is given by

$$
\overline{\mathrm{x}}_{\mathrm{B}}=\overline{\mathrm{x}}_{\mathrm{i}}+\overline{\mathrm{x}}_{\mathrm{i}} \mathrm{t}_{1}+\frac{1}{2} \overline{\mathrm{a}}_{\mathrm{d}} \mathrm{t}_{1}^{2}
$$


Inserting the expression for $t_{1}$, Eq. (4.10) becomes

$$
\overline{\mathrm{x}}_{\mathrm{B}}=\overline{\mathrm{x}}_{\mathrm{i}}-\overline{\dot{\mathrm{x}}}_{\mathrm{i}}\left(\frac{\overline{\dot{\mathrm{x}}}_{\mathrm{i}} \cdot \overline{\mathrm{i}}_{\mathrm{d}}}{\left|\overline{\mathrm{a}}_{\mathrm{d}}\right|}\right)+\frac{1}{2} \overline{\mathrm{a}}_{\mathrm{d}}\left(\frac{\overline{\dot{\mathrm{x}}}_{\mathrm{i}} \cdot \overline{\mathrm{i}}_{\mathrm{d}}}{\left|\overline{\mathrm{a}}_{\mathrm{d}}\right|}\right)^{2}
$$

Taking the component parallel to the disturbance, noting that this is the same as $-\beta$,

$$
\bar{x}_{B} \cdot \overline{\mathrm{i}}_{d}=-\beta=\bar{x}_{i} \cdot \overline{\dot{i}}_{d}-\left(\overline{\dot{x}}_{i} \cdot \overline{\dot{i}}_{d}\right)\left(\frac{\overline{\dot{x}}_{i} \cdot \overline{\mathrm{i}}_{d}}{\left|\overline{\mathrm{a}}_{d}\right|}\right)+\frac{1}{2}\left(\overline{\mathrm{a}}_{d} \cdot \overline{\dot{i}}_{d}\right)\left(\frac{\overline{\mathrm{x}}_{i} \cdot \overline{\mathrm{i}}_{d}}{\left|\overline{\mathrm{a}}_{d}\right|}\right)^{2}
$$

and reducing gives

$$
\left[\beta+\left(\bar{x}_{i} \cdot \bar{i}_{d}\right)\right]\left|\bar{a}_{d}\right|=\frac{1}{2}\left(\overline{\dot{x}}_{i} \cdot \bar{i}_{d}\right)^{2}
$$

which can be rewritten as

$$
\overline{\dot{x}}_{i} \cdot \overline{\mathrm{i}}_{d}=-\sqrt{2\left|\overline{\mathrm{a}}_{\mathrm{d}}\right|\left[\beta+\left(\overline{\mathrm{x}}_{\mathrm{i}} \cdot \overline{\mathrm{i}}_{\mathrm{d}}\right)\right]}
$$

Substituting this into Eq. (4.9) gives the final form for the time from initial attitude to point B.

$$
t_{1}=\sqrt{\frac{2\left[\beta+\left(\bar{x}_{i} \cdot \bar{i}_{d}\right)\right]}{\left|\bar{a}_{d}\right|}}
$$


Now the task is to find the time from point $B$ to point $C$, which will be called $t_{2}$. Start with the equation for the final attitude at point $\mathrm{C}$.

$$
\overline{\mathrm{x}}_{\mathrm{C}}=\overline{\mathrm{x}}_{\mathrm{B}}+\dot{\mathrm{x}}_{\mathrm{B}} \mathrm{t}_{2}+\frac{1}{2} \overline{\mathrm{a}}_{\mathrm{d}} \mathrm{t}_{2}^{2}
$$

Take the component of this along the disturbance

$$
\overline{\mathrm{x}}_{\mathrm{C}} \cdot \overline{\mathrm{i}}_{\mathrm{d}}=\left(\overline{\mathrm{x}}_{\mathrm{B}} \cdot \overline{\mathrm{i}}_{\mathrm{d}}\right)+\left(\dot{\overline{\mathrm{x}}}_{\mathrm{B}} \mathrm{t}_{2} \cdot \overline{\mathrm{i}}_{\mathrm{d}}\right)+\frac{1}{2}\left|\overline{\mathrm{a}}_{\mathrm{d}}\right| \mathrm{t}_{2}^{2}
$$

and note that this is equal to the radius of the error envelope, $r$.

$$
\bar{x}_{C} \cdot \bar{i}_{d}=r
$$

Note also that at point $\mathrm{B}$ there is no rate in the direction of the disturbance

$$
\dot{\bar{x}}_{B} \cdot \overline{\mathrm{i}}_{d}=0
$$

Simplifying Eq. (4.17) gives

$$
\mathrm{r}=-\beta+0+\frac{1}{2}\left|\overline{\mathrm{a}}_{\mathrm{d}}\right| \mathrm{t}_{2}^{2}
$$

which can be solved for $t_{2}$.

$$
\mathrm{t}_{2}=\sqrt{\frac{2(\mathrm{r}+\beta)}{\left|\overrightarrow{\mathrm{a}}_{\mathrm{d}}\right|}}
$$


Forming the equation for the final attitude from the initial attitude gives

$$
\bar{x}_{C}=\bar{x}_{i}+\bar{x}_{i}\left(t_{1}+t_{2}\right)+\frac{1}{2} \bar{a}_{d}\left(t_{1}+t_{2}\right)^{2}
$$

Finally, noting that

$$
\overline{\mathrm{x}}_{\mathrm{C}}=\mathrm{r} \cdot \overline{\mathrm{i}}_{\mathrm{d}}
$$

and solving for $\overrightarrow{\mathrm{x}}_{i}$ gives the necessary initial rate to achieve the trajectory.

$$
\overline{\mathrm{x}}_{\mathrm{i}}=\frac{1}{\mathrm{t}_{1}+\mathrm{t}_{2}}\left[\mathrm{r} \cdot \overline{\mathrm{i}}_{\mathrm{d}}-\overline{\mathrm{x}}_{\mathrm{i}}-\frac{1}{2} \overline{\mathrm{a}}_{\mathrm{d}}\left(\mathrm{t}_{1}+\mathrm{t}_{2}\right)^{2}\right]
$$

Subtracting the body rate from this gives the rate change to command.

$$
\Delta \bar{\omega}_{\mathrm{cmd}_{\text {slow }}}=\overline{\dot{x}}_{\mathrm{i}}-\bar{\omega}^{\mathrm{B}}
$$

The usefulness of this solution is contingent upon having a good estimate of the disturbance acceleration. Options for arriving at an estimate of the disturbance acceleration are presented in Chapter 5. 
The slow disturbance package is implemented with the following rules, which replace the baseline rules when activated. The rule output values are the same as in the baseline rule case.

If $\theta$ is big and $\dot{\theta}_{\text {error }}$ is small and $\alpha$ is small then jet select is good.

If $\theta$ is big and $\dot{\theta}_{\text {error }}$ is small and $\alpha$ is big then jet select is bad.

If $\theta$ is big and $\dot{\theta}_{\text {error }}$ is big and $\alpha$ is small then jet select is very bad.

If $\theta$ is big and $\dot{\theta}_{\text {error }}$ is big and $\alpha$ is big then jet select is good.

If $\theta$ is big and $\phi$ is big then jet select is bad.

If $\theta$ is big and $\phi$ is small then jet select is good.

If $\theta$ is small and $\Delta \bar{\omega}_{\text {cmd }}$ islow small and $\alpha_{\text {slow }}$ is small then jet select is good.

If $\theta$ is small and $\Delta \bar{\omega}_{\text {cmd slow }_{\text {slow }}}$ is small and $\alpha_{\text {slow }}$ is big then jet select is bad.

If $\theta$ is small and $\Delta \bar{\omega}_{\text {cmd }_{\text {slow }}}$ is big and $\alpha_{\text {slow }}$ is small then jet select is very bad.

If $\theta$ is small and $\Delta \bar{\omega}_{\text {cmd }_{\text {slow }}}$ is big and $\alpha_{\text {slow }}$ is big then jet select is good.

If $\theta$ is small and $\phi_{\text {slow }}$ is big then jet select is bad.

If $\theta$ is small and $\phi_{\text {slow }}$ is small then jet select is good.

These rules introduce several new quantities. $\Delta \bar{\omega}_{\text {cmd }_{\text {slow }}}$ is generated using Eq. (4.25). Additional logic is required to set $\Delta \bar{\omega}_{\text {cmd }_{\text {slow }}}$ to zero once the slow trajectory has been achieved and set it equal to the output of $\mathrm{Eq}$ (4.25) at the beginning of a new limit cycle. $\phi_{\text {slow }}$ and $\alpha_{\text {slow }}$ are analogous to $\phi$ and $\alpha$ except they are calculated by using the $\Delta \bar{\omega}_{\text {cmd }}$ slow vector as the desired rate change. They use the same membership functions, which are described in the baseline rule section. The membership functions for $\theta$ and $\Delta \bar{\omega}_{\text {crnd }}$ slow are shown in Figure 4.6.
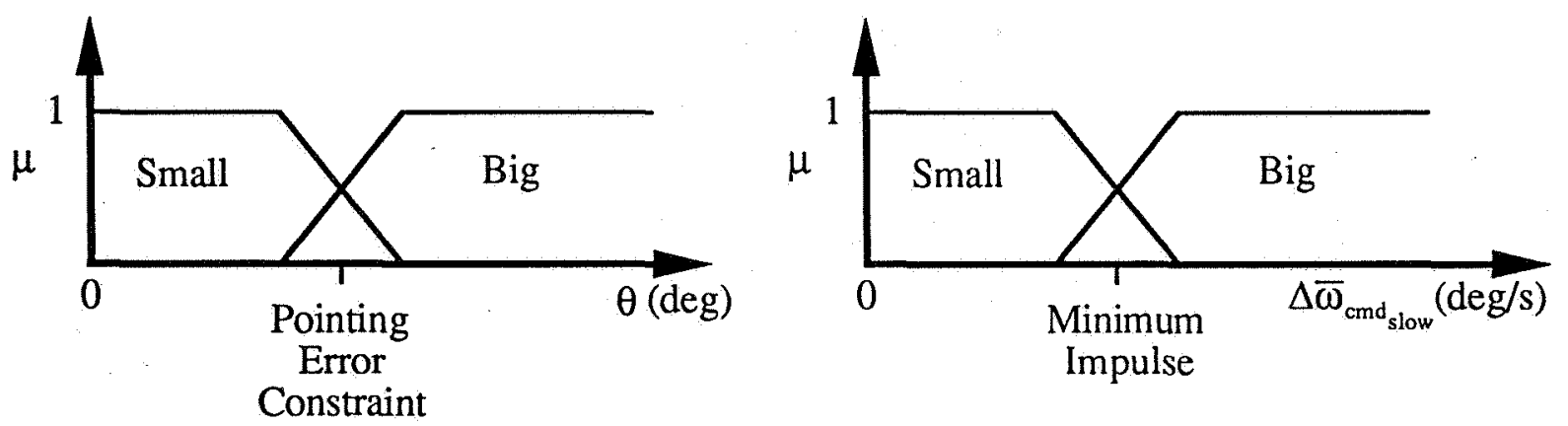

Figure 4.6. Membership Functions for $\theta$ and $\Delta \bar{\omega}_{\text {cmd }}$ slow 
The $\theta$ membership function divides the state space into two regions of operations. When $\theta$ is small the slow disturbance trajectory will be followed. As $\theta$ becomes big, the rules transition to become like the baseline rule set. Instances of big $\theta$ will cause $\theta$ to be reduced at $\dot{\theta}_{\text {cmd }}$ until $\theta$ becomes small again. As $\theta$ enters the small regime, $\Delta \bar{\omega}_{\text {cmd }_{\text {slow }}}$ becomes important. Jets will be selected which best achieve acceleration in the direction of $\Delta \bar{\omega}_{\text {cmd }}$ untow $\Delta \bar{\omega}_{\text {cnd }_{\text {slow }}}$ becomes small. At this point the slow trajectory has been achieved, $\Delta \bar{\omega}_{\text {cmd }_{\text {slow }}}$ equals zero, and the spacecraft will coast until $\theta$ begins to become big at which point the limit cycle commences again.

\subsection{Jet Limiting Rule Package for Fuel Savings}

Fuel usage is a function of burn time and the number of jets that are firing for each burn. The original rule set for evaluating possible jet selection combinations did not take into account that jet selections using more jets, also use more fuel. Therefore, the more jets a jet selection has selected for firing, the worse the selection is. The following two rules can be added to the rule sequence to implement this penalty. Good and bad are set to 1 and 0 .

If the number of jets on is big then the jet select is bad If the number of jets on is small then the jet select is good

The membership functions for small and big number of jets on are shown in Figure 4.7.

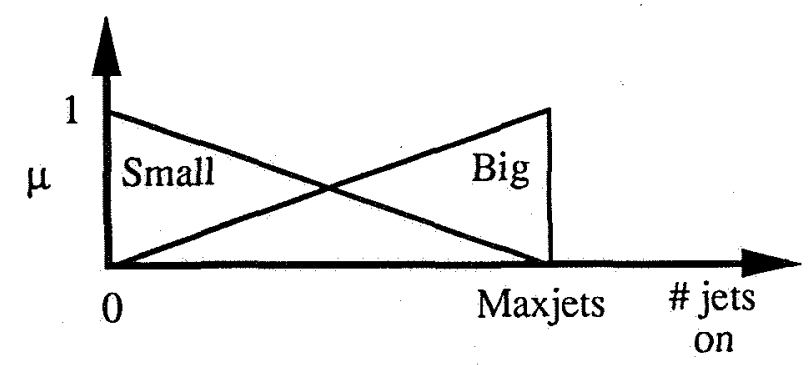

Figure 4.7. Membership Functions for Fuel Savings Package 
This provides a "knob" on the fuzzy attitude controller to emphasize the selection of fewer thrusters. Unfortunately, since jets are selected based upon the current state of the system without an attempt to find an optimal sequence of events for the future, penalizing at each jet selection the number of jets selected for firing can actually lead to worse performance over the long run if the penalty is too great. The output weight of this rule package can not be raised indefinitely in a quest for improved performance.

\subsection{Anti-Chatter Rule Package}

Jet chattering is a problem because it decreases the operational lifetime of the jets. One situation in which jet chatter can occur is during an acceleration or a deceleration burn. Because we are using a control strategy that selects thrusters every sample time, it is possible the jet selection will be changed often, especially during long burns such as occur at the beginning and end of attitude maneuvers.

The intent of this rule package is to penalize jet selections having a large number of on/off or off/on switches from the previous jet selection. For example, to go from a jet select of three jets to another jet select of three different jets implies a total of six on and off switches. This penalty can be implemented with the following rules. Good and bad are set to 1 and 0 , respectively.

If the number of on/off switches is big then jet select is bad If the number of on/off switches is small then jet select is good

The membership functions for big and small number of jet switches are shown in Figure 4.8. 


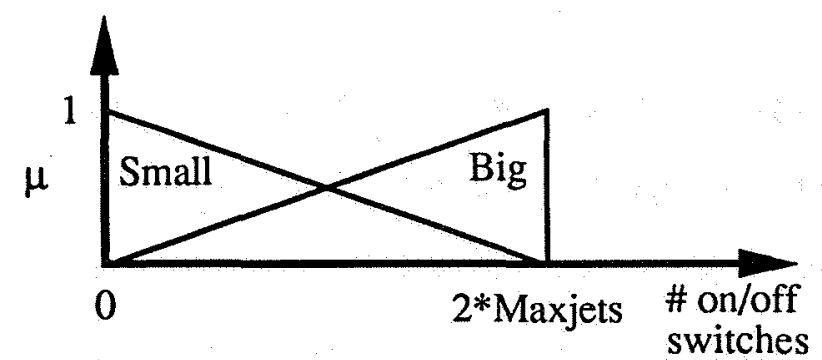

Figure 4.8. Membership Functions for Anti-Chatter Rule Package

As in the previous rule package, care should be taken when setting the output weight of this rule package. If the fuzzy attitude controller is inhibited from switching jets on and off to too great an extent by a large output weighting on this rule package, performance may actually be worse, causing an increase in jet on/off switches over the course of the entire maneuver or attitude hold.

\subsection{Anti-Flex Rule Package}

The anti-flex rule package is a set of additional rules which can be added if the spacecraft attitude can be affected by flexible appendages in a frequency range within the controller bandwidth. The premise of these rules is to penalize jet combinations that will add energy to the flexible modes and promote those which damp them. This rule package relies upon an estimate of the total angular rate due to the flexible modes, $\bar{\omega}^{\text {flex }}$. The method for achieving this estimate is described in Chapter 5.

The linear model of the flexible dynamics is used to determine the flex rate change due to a jet firing, $\Delta \bar{\omega}_{\text {jet }}^{\text {flex }}$. First arrange the model, Eq (3.3), to obtain the transfer function from the jet-on vector $\overline{\mathrm{u}}$ to the flex rates at the location of the rate sensor (or about the base-body c.g. for a rigid base-body). 


$$
\begin{aligned}
& {\left[\begin{array}{c}
\overline{\mathrm{w}} \\
\overline{\overline{\mathrm{w}}}
\end{array}\right]=\overbrace{\left[\begin{array}{cc}
0 & \mathrm{I} \\
-\omega^{2} & -2 \zeta \omega
\end{array}\right]}^{A_{\text {nax }}}\left[\begin{array}{l}
\overline{\mathrm{w}} \\
\overline{\mathrm{w}}
\end{array}\right]+\overbrace{\left[\begin{array}{c}
0 \\
B_{\text {jels }}
\end{array}\right] \overline{\mathrm{u}}}^{\mathrm{B}_{\text {anx }}}}
\end{aligned}
$$

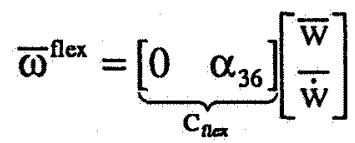

Then calculate the discrete time equivalent of the $B_{\text {flex }}$ matrix using the zero order hold.

$$
\Gamma_{\text {flex }}=\int_{0}^{T} e^{A_{\text {nex }} \sigma} d \sigma B_{\text {nex }}=\sum_{n=1}^{\infty} \frac{\Delta t^{n} A_{\text {flex }}^{n-1} B_{\text {fex }}}{n !}
$$

where $\Delta \mathrm{t}$ is the control system sample interval. Then $\Delta \bar{\omega}_{\mathrm{jed}_{1}}^{\mathrm{flex}}$, the flex rate change due to the ith jet firing is

$$
\Delta \bar{\omega}_{\mathrm{jet}_{\mathrm{i}}}^{\mathrm{flex}}=\mathrm{C}_{\text {flex }} \Gamma_{\mathrm{flex}} \overline{\mathrm{u}} ; \overline{\mathrm{u}}_{\mathrm{j}=\mathrm{i}}=1, \overline{\mathrm{u}}_{\mathrm{j} \neq \mathrm{i}}=0
$$

Since the modal energies cannot be directly measured, the actual quantity being driven to zero is $\bar{\omega}^{\text {flex }}$. The 2-norm of $\bar{\omega}^{\text {flex }}$, denoted by $\bar{\omega}_{\mathrm{L}_{2}}^{\text {flex }}$ and $\left(\left\|\bar{\omega}^{\text {flex }}+\Delta \bar{\omega}_{\text {jet }}^{\text {flex }}\right\|-\left\|\bar{\omega}^{\text {flex }}\right\|\right)$, denoted by $\Delta \bar{\omega}_{\mathrm{L}_{2}}^{\text {flex }}$ which is the measure of how much the jet selection being evaluated will increase $\bar{\omega}_{\mathrm{L}_{2}}^{\text {flex }}$, are calculated for the various jet combinations and used in the following rules. The rule outputs very bad, bad, slightly bad, slightly good, good, and very good are assigned the values $-1,-.5,-.1, .1, .5$, and 1 , respectively. 
If $\bar{\omega}_{\mathbf{L}_{2}}^{\text {flex }}$ is big and $\Delta \bar{\omega}_{\mathbf{L}_{2}}^{\text {flex }}$ is positive big then jet select is very bad If $\bar{\omega}_{L_{2}}^{\text {flex }}$ is big and $\Delta \bar{\omega}_{L_{2}}^{\text {flex }}$ is positive small then jet select is bad If $\bar{\omega}_{\mathbf{L}_{2}}^{\text {flex }}$ is big and $\Delta \bar{\omega}_{\mathbf{L}_{2}}^{\text {flex }}$ is negative small then jet select is good If $\bar{\omega}_{\mathbf{L}_{2}}^{\text {flex }}$ is big and $\Delta \bar{\omega}_{\mathbf{L}_{2}}^{\text {flex }}$ is negative big then jet select is very good If $\bar{\omega}_{\mathrm{L}_{2}}^{\text {flex }}$ is small and $\Delta \bar{\omega}_{\mathrm{L}_{2}}^{\text {flex }}$ is positive big then jet select is bad If $\bar{\omega}_{\mathrm{L}_{2}}^{\text {flex }}$ is small and $\Delta \bar{\omega}_{\mathrm{L}_{2}}^{\text {flex }}$ is positive small then jet select is slightly bad If $\bar{\omega}_{L_{2}}^{\text {flex }}$ is small and $\Delta \bar{\omega}_{L_{2}}^{\text {flex }}$ is negative small then jet select is slightly good If $\bar{\omega}_{\mathrm{L}_{2}}^{\text {flex }}$ is small and $\Delta \bar{\omega}_{\mathrm{L}_{2}}^{\text {flex }}$ is negative big then jet select is good

These rules place more weight upon the importance of damping the flexible modes when the magnitude of $\bar{\omega}^{\text {flex }}$ is big. When the magnitude is small, damping the flexible modes is not a priority; however, it is still important to favor jet selections which will not excite the flexible modes. The ability to select jets which will avoid excitation of flexible modes is a significant advantage over more traditional means of dealing with flexible dynamics although these controllers may come with a cost of increased thruster firings. Controllers that filter the flexible modes out of the measurement, may maintain stability, but can lead to significant excitation of the flex dynamics.

The relevant membership functions are shown in Figure 4,9. The first membership functions are sized according to the rate error constraint. The membership functions for $\Delta \bar{\omega}_{L_{2}}^{\text {flex }}$ are sized according to the expected range of $\Delta \bar{\omega}_{L_{2}}^{\text {flex }}, \sigma$, as calculated in Eq. (4.29).
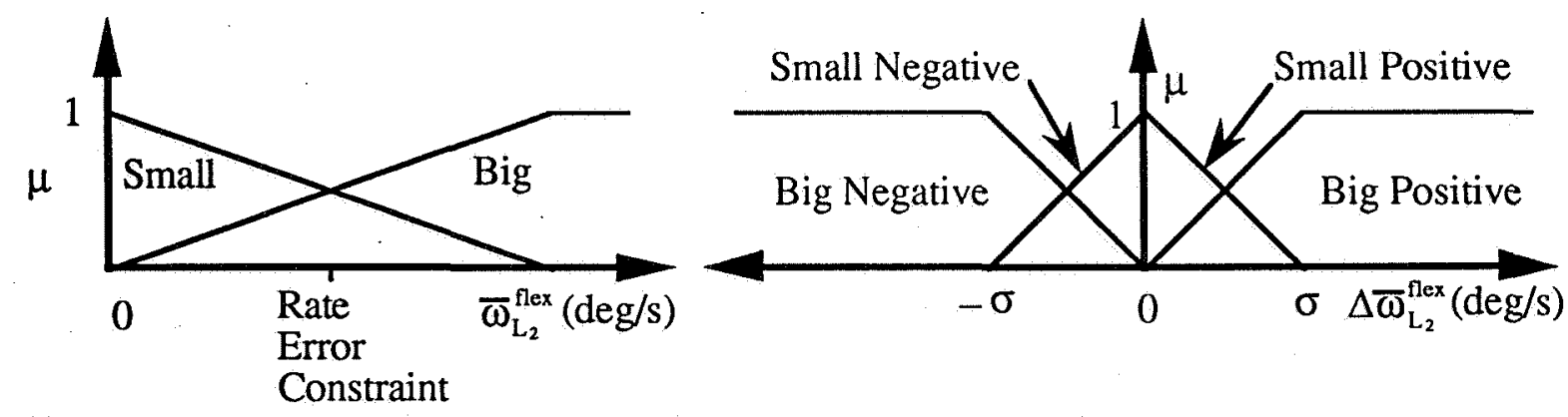

Figure 4.9. Membership Functions for Anti-Flex Rule Package 


$$
\begin{gathered}
\sigma=\operatorname{Maxjets} \frac{\sum_{i=1}^{\text {njets }}\left\|\Delta \bar{\omega}_{\text {jet }_{i}}^{\text {flex }}\right\|}{\text { njets }} \\
\text { njets }=\text { number of available jets }
\end{gathered}
$$

It is unlikely that the available flex models will be highly accurate; however, this rule package is robust to both modal frequency shifts and amplitude variations. The fuzzy controller does not care at what frequency a mode is oscillating, only the current direction of the modal oscillation is important. This information is provided by $\bar{\omega}^{\text {flex }}$, which allows jets to be selected to oppose its rate. Amplitude variation is also handled well, because jets are chosen to avoid directions that excite the flexible rates. Amplitude modeling error will not cause worse performance. Performance will simply reflect what is achievable for the true system. This rule package is not robust to large changes in the direction jets map into the flexible modes. Errors of this sort could cause the controller to select a jet combination which it thought would damp the flexible modes but actually excites them. 



\section{Chapter 5}

\section{State Estimator}

The fuzzy attitude controller requires the accurate estimation of several signals. The slow disturbances rule package performs a trajectory calculation based upon the slow disturbance acceleration. The quality of the trajectory is directly related to the accuracy with which the slow disturbance acceleration can be estimated. If the anti-flex rule package is used, it requires estimation of the body rates due to flexible modes. Poor estimation of these rates can cause the anti-flex rules to pick jets which will excite rather than damp flexible modes.

This chapter first presents means for estimating the slow disturbance acceleration and then illustrates two approaches for estimating the body rates due to flexible modes.

\subsection{Slow Disturbance Acceleration Estimation}

On spacecraft where the predicted rate change due to a jet firing is accurate, the slow disturbance acceleration $\overline{\mathrm{a}}_{\mathrm{d}}$ can be estimated using a simple filter which takes as input the difference between the current rate measurement and the previous rate measurement, and feedforward of the expected rate change due to jet firings. Here $\mathrm{T}$ is the sample time and $\mathrm{K}$ is the filter gain chosen to be small enough such that the filter rolls off to attenuate high frequency disturbances such as flexible body dynamics. 


$$
\bar{a}_{d n}=\bar{a}_{d n-1}-K\left[\bar{a}_{d n-1}-\frac{\bar{\omega}_{n}^{B}-\left(\bar{\omega}_{n-1}^{B}+\Delta \bar{\omega}_{j \mathrm{jl}}\right)}{T}\right]
$$

In order to size $\mathrm{K}$, first rewrite the difference equation replacing the residual above with $\bar{\alpha}_{\text {residualn }}^{\text {B }}$.

$$
\bar{a}_{d_{n}}=\bar{a}_{d_{n-1}}-K \bar{a}_{d_{n-1}}+K \bar{\alpha}_{\text {residual }}^{B}
$$

Then transform into the $\mathrm{Z}$ domain.

$$
\bar{A}_{d}(z)=z^{-1} \bar{A}_{d}(z)-K z^{-1} \bar{A}_{d}(z)+K \bar{\alpha}_{\text {residual }}^{B}(z)
$$

Solve for the transfer function in the $\mathrm{Z}$ domain

$$
\frac{\bar{A}_{d}(z)}{\bar{\alpha}_{\text {residual }}^{\mathrm{B}}(\mathrm{z})}=\frac{\mathrm{K}}{1+\mathrm{z}^{-1}(\mathrm{~K}-1)}=\frac{\mathrm{Kz}}{\mathrm{z}+\mathrm{K}-1}
$$

and transform into the Laplace domain.

$$
\frac{\overline{\mathrm{A}}_{\mathrm{d}}(\mathrm{s})}{\overline{\boldsymbol{\alpha}}_{\text {residual }}^{\mathrm{B}}(\mathrm{s})}=\frac{\mathrm{K}}{\mathrm{s}-\frac{\ln (1-\mathrm{K})}{\mathrm{T}}}=\frac{\mathrm{K}}{\mathrm{s}+\mathrm{a}} ; \mathrm{a}=-\frac{\ln (1-\mathrm{K})}{\mathrm{T}}
$$

Choose the pole location, -a, such that $\bar{\alpha}_{\text {residualn }}^{\mathrm{B}}$ is attenuated to a level below the magnitude of the expected slow disturbance acceleration at the frequency of any higher frequency disturbance. Then set the filter gain, $\mathrm{K}$, according to Eq. (5.6).

$$
\mathrm{K}=1-\mathrm{e}^{-\mathrm{aT}}
$$

In many cases the first order filter shown in Eq. (5.1) may not provide enough attenuation at frequencies of non-slow disturbance accelerations while maintaining sufficient 
bandwidth to allow a timely estimate of the slow disturbance acceleration. The solution to this problem is to first pass the residual through a higher order filter as in Eq (5.7).

$$
\begin{aligned}
\bar{x}_{\mathrm{n}+1}^{\text {filt }} & =A_{\text {filt }} \bar{x}_{n}^{\text {filt }}+B_{\text {filt }}\left[\frac{\bar{\omega}_{\mathrm{n}}^{B}-\left(\bar{\omega}_{n-1}^{B}+\Delta \bar{\omega}_{\text {jet }_{n}}\right)}{T}\right] \\
y_{n} & =C_{\text {filt }} \bar{x}_{n}^{\text {filt }} \\
\bar{a}_{d n} & =\bar{a}_{d_{n-1}}-K\left(\bar{a}_{d_{n-1}}-y_{n}\right)
\end{aligned}
$$

If the predicted rate changes due to jet firings are not accurate, more advanced techniques may be required such as $\mu$-synthesis to build in robustness to the poor jet model or an adaptive approach to learn a better jet model. Measurement transport delays may also cause a timing mismatch between the predicted or feedforward rate change and the measurement rate. Once the software and hardware is in place for a particular vehicle, these delays can be determined and accounted for by an appropriate delay of the feedforward term.

\subsection{Flexible Rate Estimator}

The approach taken to estimate $\bar{\omega}^{\text {flex }}$ is first to estimate the rigid body rate $\bar{\omega}^{\text {rigid }}$ and subtract this from the measured body rate, $\bar{\omega}^{\mathrm{B}}$. It is important that the estimate of $\bar{\omega}^{\text {rigid }}$ have good transient behavior, otherwise the estimate of $\bar{\omega}^{\text {flex }}$ may be in the opposite direction from the actual rate. This dictates using a model based estimator, allowing predicted rate changes of jet firings to be fed forward.

Two different approaches were taken for the design of this estimator. The first uses linear optimal estimation, [12]. The plant to be observed is:

$$
\begin{aligned}
\dot{x} & =A x+B \dot{\alpha}_{j c t}+L \xi \\
\omega^{\text {rigid }} & =C x+\theta
\end{aligned}
$$


where $A=0, B=1, C=1$, and $L=1$. This is a simple integrator representing the transfer function from jet acceleration to rate change, where $\xi$, the process noise is gaussian, zeromean, and white with constant intensity matrix, $\Xi . \theta$, the measurement noise, is also gaussian, zero-mean, and white with constant intensity matrix, $\Theta$, and is assumed to be independent of the process noise. The linear optimal observer (or Kalman filter) based upon this plant is

$$
\begin{aligned}
\hat{\dot{x}} & =A \hat{x}+B \alpha_{\text {jet }}+H\left(\omega^{B}-\hat{\omega}^{\text {rigid }}\right) \\
\hat{\omega}^{\text {rigid }} & =C \hat{x}
\end{aligned}
$$

where the filter gain, $\mathrm{H}$, is found from the solution to the filter algebraic Riccati equation.

$$
\begin{aligned}
& 0=A \Sigma+\Sigma A^{\mathrm{T}}+L \Xi \mathrm{L}^{\mathrm{T}}-\Sigma \mathrm{C}^{\mathrm{T}} \Theta^{-1} \mathrm{C} \Sigma \\
& H=\Sigma \mathrm{C}^{\mathrm{T}} \Theta^{-1}
\end{aligned}
$$

The closed loop Kalman filter becomes

$$
\begin{aligned}
& \hat{\dot{x}}=\left[\begin{array}{ll}
A-H C
\end{array}\right] \hat{x}+\left[\begin{array}{ll}
B & H
\end{array}\right]\left[\begin{array}{l}
\alpha_{j e l} \\
\omega^{B}
\end{array}\right] \\
& \hat{\omega}^{\text {rigid }}=C \hat{x}
\end{aligned}
$$

The two design parameters available are $\Xi$ and $\Theta$. If the plant model does not capture all of the relevant dynamics then the elements of $\Xi$ can be increased. If however, the measurement is known to be badly corrupted, the elements of $\Theta$ can be increased. For this design the latter is true. The flexible modes are corrupting the measurement of the rigid body rate. 
If the frequencies at which the measurement is corrupted are known the filter can be improved through the use of a shaping filter representing the noise. In this case the measurement is corrupted over the frequencies of the flexible modes. Now, suppose the measurement noise is the sum of a noise term due to the flexible modes and a term due to the actual sensor noise which is assumed to be gaussian, zero-mean, and white.

$$
\theta=\theta_{\text {flex }}+\theta_{\text {sensor }}
$$

$\theta_{\text {flex }}$ can be generated by passing gaussian, zero-mean, white noise, call it $\psi$ with constant intensity matrix $\Psi$, through a band pass filter, attenuating $\psi$ at frequencies unaffected by the flexible modes.

$$
\begin{aligned}
\overline{\dot{x}}_{w} & =A_{w} \bar{x}_{w}+B_{w} \psi \\
\theta_{\text {flex }} & =C_{w} \bar{x}_{w}
\end{aligned}
$$

The plant model becomes

$$
\begin{aligned}
\dot{x} & =A x+B \alpha_{j e t}+L \xi \\
\omega^{B} & =C x+\theta_{\text {flex }}+\theta_{\text {scnsor }}
\end{aligned}
$$

The dynamics of Eq. (5.13) augment those of Eq. (5.14) to obtain the filter design model.

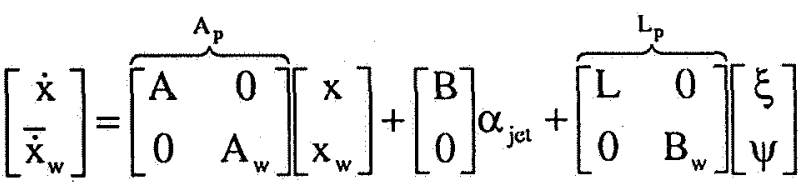

$$
\begin{aligned}
& \omega^{B}=\underbrace{\left[\begin{array}{ll}
C & C_{w}
\end{array}\right]}_{C_{p}}\left[\begin{array}{l}
x \\
x_{w}
\end{array}\right]+\theta_{\text {sensor }}
\end{aligned}
$$


Now both the process noises are combined into a new process noise vector with the constant intensity matrix

$$
\Xi^{\prime}=\left[\begin{array}{cc}
\Xi & 0 \\
0 & \Psi
\end{array}\right]
$$

The solution for the Kalman filter gain vector is now the following, where subscript $p$ denotes the augmented state matrices from Eq. (5.15).

$$
\begin{aligned}
& 0=\mathrm{A}_{\mathrm{p}} \Sigma+\Sigma \mathrm{A}_{\mathrm{p}}^{\mathrm{T}}+\mathrm{L}_{\mathrm{p}} \Xi^{\prime} \mathrm{L}_{\mathrm{p}}^{\mathrm{T}}-\Sigma \mathrm{C}_{\mathrm{p}}^{\mathrm{T}} \Theta_{\text {sensor }}^{-1} \mathrm{C}_{\mathrm{p}} \Sigma \\
& \mathrm{H}=\Sigma \mathrm{C}_{\mathrm{p}}^{\mathrm{T}} \Theta_{\text {sensor }}^{-1}
\end{aligned}
$$

which can be used to form the Kalman filter.

$$
\begin{aligned}
& {\left[\begin{array}{c}
\hat{\dot{x}} \\
\dot{x}_{w}
\end{array}\right]=\left[\begin{array}{cc}
A-H_{x} C & -H_{x} C_{w} \\
-H_{w} C & -H_{w} C_{w}
\end{array}\right]\left[\begin{array}{c}
\hat{x} \\
x_{w}
\end{array}\right]+\left[\begin{array}{cc}
B & H_{x} \\
\overline{0} & H_{w}
\end{array}\right]\left[\begin{array}{c}
\alpha_{\text {jet }} \\
\omega^{B}
\end{array}\right]} \\
& \hat{\omega}^{\text {rigid }}=\left[\begin{array}{ll}
C & 0
\end{array}\right]\left[\begin{array}{c}
\hat{x} \\
x_{w}
\end{array}\right]
\end{aligned}
$$

The second approach used to estimate $\bar{\omega}^{\text {rigid }}$ utilized a model based observer with the addition of frequency weighted gains. As before the initial observer was of the form shown in Eq (5.9). The transient behavior of this observer proved to be inadequate. With $\mathrm{H}$ set small enough to attenuate the frequencies of the flexible modes, the steady state error between $\bar{\omega}^{\text {rigid }}$ and $\hat{\bar{\omega}}^{\text {rigid }}$ decayed too slowly. The solution was to use frequency weighting, placing more importance upon the low frequency content of the residual, $\left(\omega^{B}-\hat{\omega}^{\text {rigid }}\right)$. The augmented estimator written in closed loop form looks like 


$$
\begin{aligned}
& {\left[\begin{array}{c}
\hat{\dot{x}} \\
\dot{z} \\
\ddot{z}
\end{array}\right]=\left[\begin{array}{ccc}
\mathrm{A}-\mathrm{H}_{\mathrm{x}} \mathrm{C} & -\mathrm{H}_{\mathrm{z} 1} & -\mathrm{H}_{\mathrm{z} 2} \\
0 & 0 & 1 \\
-\omega_{\text {fill }}^{2} \mathrm{C} & -\omega_{\text {filt }}^{2} & -2 \zeta \omega_{\text {filt }}
\end{array}\right]\left[\begin{array}{l}
\hat{\mathrm{x}} \\
\mathrm{z} \\
\dot{\mathrm{z}}
\end{array}\right]+\left[\begin{array}{cc}
\mathrm{B} & \mathrm{H}_{\mathrm{x}} \\
0 & 0 \\
0 & \omega_{\text {filt }}^{2}
\end{array}\right]\left[\begin{array}{l}
\alpha_{\text {jet }} \\
\omega^{\mathrm{B}}
\end{array}\right]} \\
& \hat{\omega}^{\text {igid }}=\left[\begin{array}{lll}
\mathrm{C} & 0 & 0
\end{array}\right]\left[\begin{array}{c}
\hat{\mathrm{x}} \\
\mathrm{z} \\
\dot{\mathrm{z}}
\end{array}\right]
\end{aligned}
$$

The second order low-pass filter, with roll off frequency $\omega_{\text {fitt }}$ and damping ratio $\zeta$ set for critical damping, allows the gain on the residual to be increased at low frequencies through the appropriate setting of $\mathrm{H}_{\mathrm{z} 1}$ and $\mathrm{H}_{\mathrm{z} 2}$.

Suitable values for the gain vector $\mathrm{H}$ and $\omega_{\text {filt }}$ can be determined using the Matlab function "fmins". Fmins, which employs a Simplex search method, can be set up to search over the space of possible $\mathrm{H}$ and $\omega_{\text {filt }}$ looking to minimize a cost function. The cost function in this case is the RMS of the estimation error, $\left(\omega^{\text {rigid }}-\hat{\omega}^{\text {rigid }}\right)$, summed over time for several simulated maneuvers. It is important to use more than one maneuver with fmins, otherwise the solution fmins finds will work excellently for the one case it was presented but not so well for others. Setting the cost function to be the sum of the RMS of $\left(\omega^{\text {igid }}-\hat{\omega}^{\text {rigid }}\right)$ over three disparate maneuvers should be sufficient. This approach is contingent upon having access to $\omega^{\text {rigid }}$ in the simulation.

After finding the estimate of the rigid body rates, $\hat{\bar{\omega}}^{\text {rigid }}$, the flexible rate estimate is just the difference between $\hat{\bar{\omega}}^{\text {rigid }}$ and the measured body rates.

$$
\hat{\bar{\omega}}^{\text {flex }}=\bar{\omega}^{\mathrm{B}}-\hat{\bar{\omega}}^{\text {rigid }}
$$





\section{Chapter 6}

\section{Space Shuttle Characteristics}

To demonstrate the fuzzy attitude controller, the rules described in Chapter 4 will be applied to the space shuttle orbiter in its operational environment. Comparisons between fuzzy control performance and the orbiter flight control system (FCS) performance during typical on-orbit operations will be shown in the following chapter. All simulations are done using Draper Laboratory Interactive On-Orbit Simulator (IOS) [13].

This chapter briefly describes the shuttle FCS and its operational environment.

\subsection{Mass Properties}

The mass properties of the space shuttle are described by three different parameters: the mass, the location of the center of gravity (c.g.) with respect to the orbiter structural reference system (OSRS) (Figure 6.1), [14], and the inertia matrix about the c.g. in OSRS. The following mass properties are used for all orbiter alone cases presented in this thesis. 


$$
\text { orbiter mass(slugs) }=6694.4816
$$

$$
\begin{aligned}
\text { orbiter c.g.(ft) } & =\left[\begin{array}{c}
91.292 \\
-.025 \\
31.083
\end{array}\right] \\
\text { orbiter inertia matrix }\left(-\int\right)\left(\text { slug } \mathrm{ft}^{2}\right) & =\left[\begin{array}{ccc}
.933 \mathrm{e} 6 & .336 \mathrm{e} 4 & -.212 \mathrm{e} 6 \\
.336 \mathrm{e} 4 & .713 \mathrm{e} 7 & -.444 \mathrm{e} 3 \\
-.212 \mathrm{e} 6 & -.444 \mathrm{e} 3 & .746 \mathrm{e} 7
\end{array}\right]
\end{aligned}
$$

The $-\int$ symbol implies that the negative integral convention was used to determine the products of inertia (i.e. $I_{x y}=-\int(x y) d m$ ).

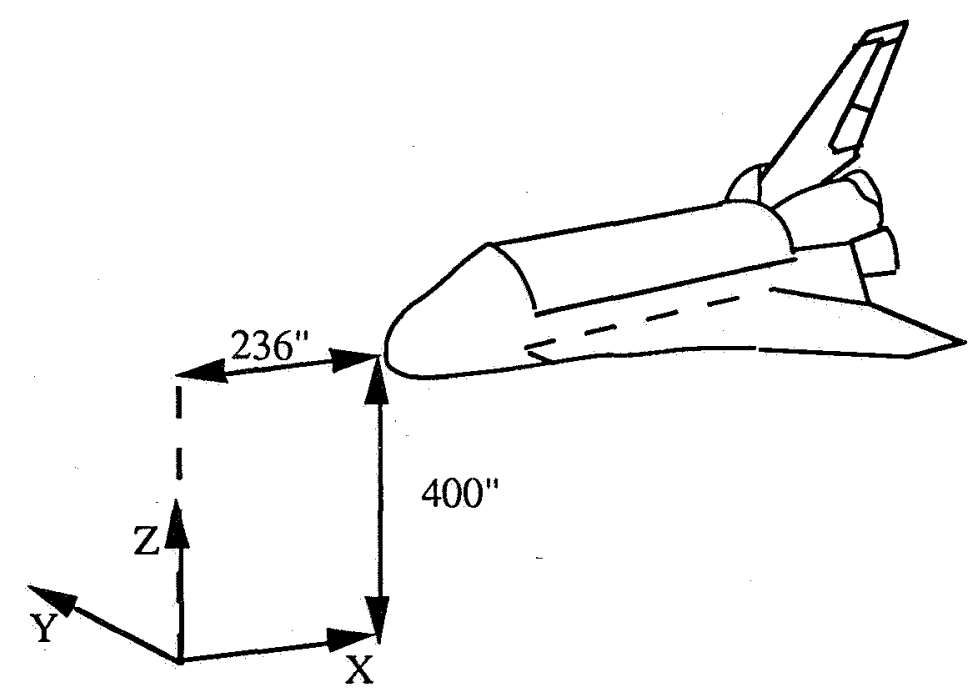

Figure 6.1. OSRS Coordinate System

\subsection{Actuators}

The space shuttle is equipped with two different hypergolic liquid bipropellant thruster systems for performing attitude maneuvers. These systems are the vernier and the primary jets (table 6.1). There are six vernier and 38 primary jets located about the space shuttle in 14 groups as shown in Figure 6.2. Each jet within a group provides essentially the same torque about the space shuttle c.g. For rotation control, only one jet from a group may be selected for firing at a time. This jet is known as the "priority one jet". The priority one jets can be changed from mission to mission in order to distribute jet wear. The vernier 
jets with their smaller thrust, 24lbs, versus the 870lbs thrust primary jets, can be used for operations requiring tight pointing requirements or smaller jet impulses, Hattis[15].

Table 6.1. Jet Performance Specifications

\begin{tabular}{||l|r|r|}
\hline \hline & \multicolumn{1}{|c|}{ Primary } & \multicolumn{2}{|c|}{ Vernier } \\
\hline Thrust (lbf) & 870 & 24 \\
\hline ISP (s) & 280 & 265 \\
\hline Duty Cycle Life & 20,000 & 500,000 \\
\hline On-time Life (s) & 12,800 & 125,000 \\
\hline
\end{tabular}

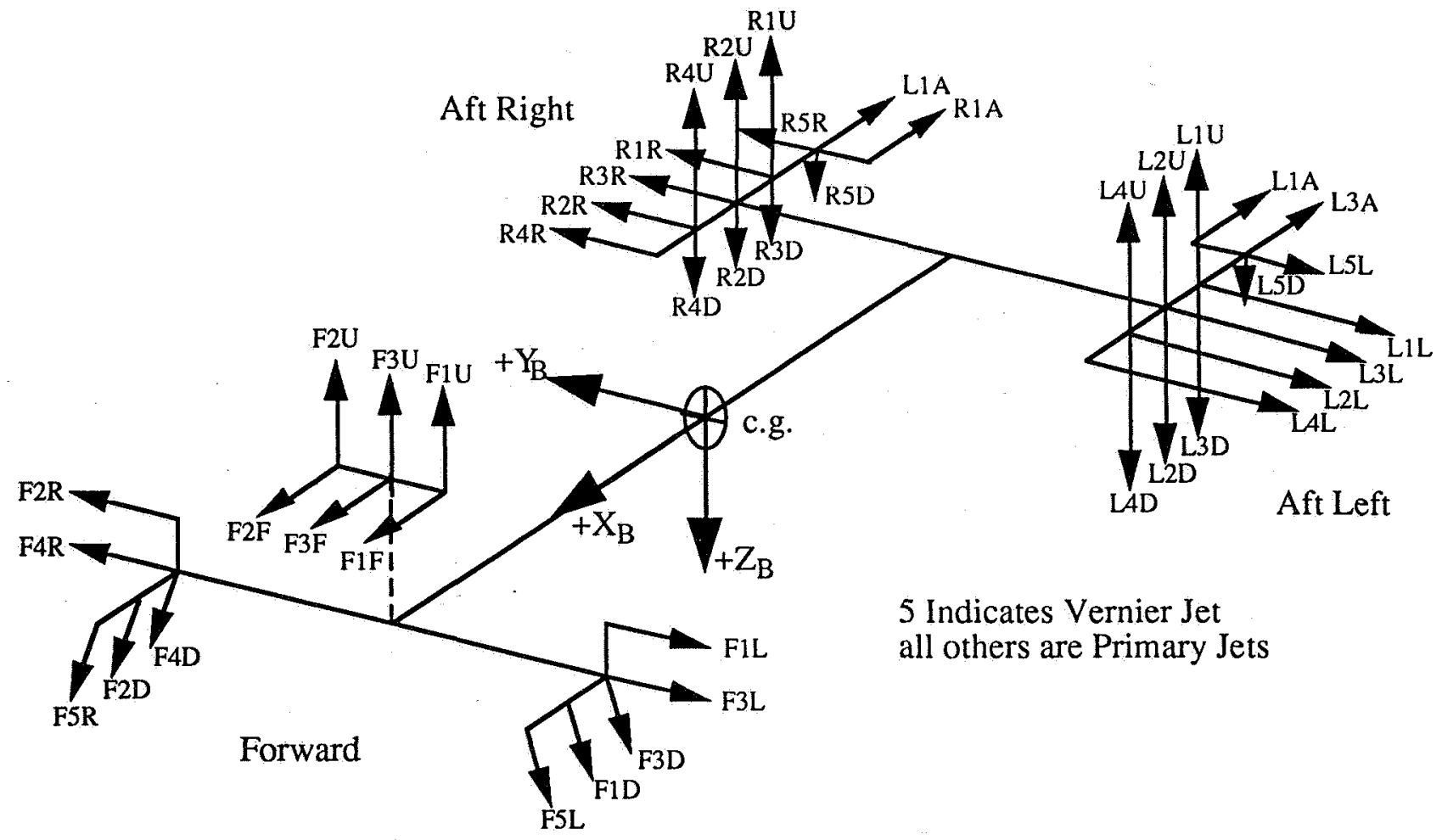

Figure 6.2. Space Shuttle Jet Locations

\subsection{Disturbances}

Table 6.2 lists typical magnitudes for the major disturbances which act upon the space shuttle while on orbit, Appleby[16].

Table 6.2. Magnitude of Disturbances Acting on Space Shuttle

\begin{tabular}{|l|c|}
\hline \multicolumn{1}{|c|}{ Source } & Acceleration $\left(\mathrm{deg} / \mathrm{s}^{2}\right)$ \\
\hline Gravity Gradient & $10^{-4}$ \\
\hline Aerodynamic & $10^{-5}-10^{-4}$ \\
\hline Payload Dynamics & $\sim 10^{-2}$ \\
\hline
\end{tabular}




\subsection{Space Shuttle-GRO System}

In order to study attitude control of the space shuttle using alternate mass properties, and controlling attitude in the presence of flexibility, a model of the space shuttle with the gamma ray observatory deployed on the shuttle remote manipulator system (SRMS) extended above the payload bay (Figure 6.3) was developed.

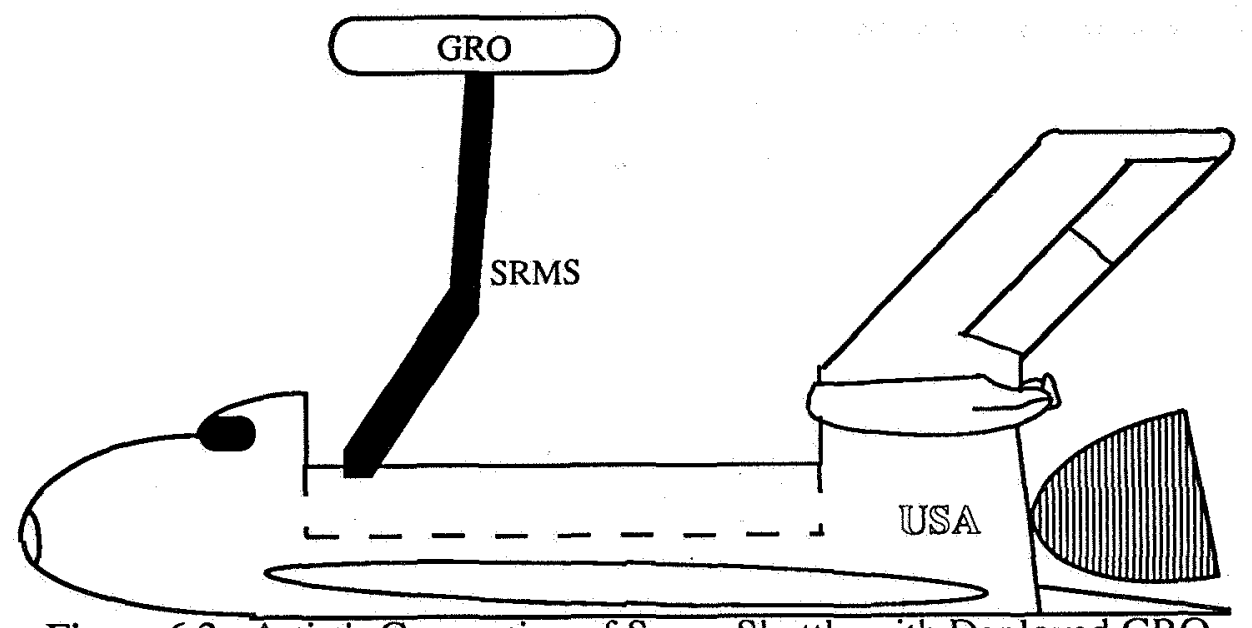

Figure 6.3. Artist's Conception of Space Shuttle with Deployed GRO

\subsubsection{Combined Mass Properties}

The combined mass properties of the space shuttle and the GRO in the above payload bay configuration are as follows. The reference frame is the OSRS.

$$
\begin{aligned}
& \text { orbiter mass(slugs) }=7278.4726 \\
& \text { orbiter c.g. }(f t)=\left[\begin{array}{c}
88.247 \\
.898 \\
38.882
\end{array}\right] \\
& \text { orbiter inertia matrix }\left(-\int\right)\left(\text { slug } \mathrm{ft}^{2}\right)=\left[\begin{array}{ccc}
3.524 \mathrm{e} 6 & -15.094 \mathrm{e} 4 & 1.139 \mathrm{e} 6 \\
-15.094 \mathrm{e} 4 & 1.041 \mathrm{e} 7 & 298.509 \mathrm{e} 3 \\
1.139 \mathrm{e} 6 & 298.509 \mathrm{e} 3 & .825 \mathrm{e} 7
\end{array}\right]
\end{aligned}
$$




\subsubsection{Model of Flexibility}

A linear model of the flexible SRMS with the GRO deployed as described above was created using a massless arm assumption as specified in Barrows[17]. The transfer function is from the 44 orbiter jets to flex-body accelerations seen at the orbiter center of gravity, Eq. (6.1).

$$
\begin{aligned}
& {\left[\begin{array}{c}
\overline{\mathrm{w}} \\
\overline{\mathrm{w}}
\end{array}\right]=\left[\begin{array}{cc}
0 & \mathrm{I} \\
-\omega^{2} & -2 \zeta \omega
\end{array}\right]\left[\begin{array}{c}
\overline{\mathrm{w}} \\
\overline{\mathrm{w}}
\end{array}\right]+\left[\begin{array}{c}
0 \\
\mathrm{~B}_{\text {jets }}
\end{array}\right] \overline{\mathrm{u}} } \\
& \bar{\alpha}_{\text {flex }}=\left[\begin{array}{ll}
0 & \alpha_{36}
\end{array}\right]\left[\begin{array}{cc}
0 & \mathrm{I} \\
-\omega^{2} & -2 \zeta \omega
\end{array}\right]\left[\begin{array}{c}
\overline{\mathrm{w}} \\
\overline{\mathrm{w}}
\end{array}\right]+\left[\begin{array}{ll}
0 & \alpha_{36}
\end{array}\right]\left[\begin{array}{c}
0 \\
\mathrm{~B}_{\text {jels }}
\end{array}\right] \overline{\mathrm{u}} \\
& \overline{\mathrm{u}}= \text { Jet on vector }(1=\text { on }) \\
& \overline{\mathrm{w}}= \text { linear and angular SRMS tip displacements } \\
& \omega= \text { SRMS natural frequencies (6 modes) } \\
& \zeta= \text { damping ratio } \\
& \alpha_{36}= \text { map from SRMS tip displacement states to } \\
& \text { rotational degrees of freedom about orbiter c.g. }
\end{aligned}
$$

The modal frequencies are shown in table 6.3. All modes have $2 \%$ damping.

Table 6.3. Modal Frequencies of Combined Space Shuttle-GRO System

\begin{tabular}{||c|r|}
\hline \multicolumn{2}{|c|}{ Modal Frequencies(rad/s) } \\
\hline \hline Mode 1 & .268 \\
\hline Mode 2 & .316 \\
\hline Mode 3 & .671 \\
\hline Mode 4 & .759 \\
\hline Mode 5 & 1.116 \\
\hline Mode 6 & 3.638 \\
\hline
\end{tabular}

\subsection{Current Shuttle Flight Control System}

The space shuttle flight control system divides the attitude control problem into several tasks, each of which is handled by a different module (Figure 6.4). The input to the entire system is a quaternion which reflects the shuttle's attitude with respect to the inertial frame $\mathrm{M} 50$, an earth centered frame with the $\mathrm{z}$-axis aligned with the earth's polar axis and the $\mathrm{x}$ - 
axis defined to point in the direction which went from earth center to sun center on Jan. 1, 1950. This quaternion is fed into the universal pointing, the auto-maneuver, and the state estimator modules. The other modules are the phase plane and jet select modules. The primary cycle rate of the autopilot is $12.5 \mathrm{~Hz}$ ( $80 \mathrm{msec}$ sample).

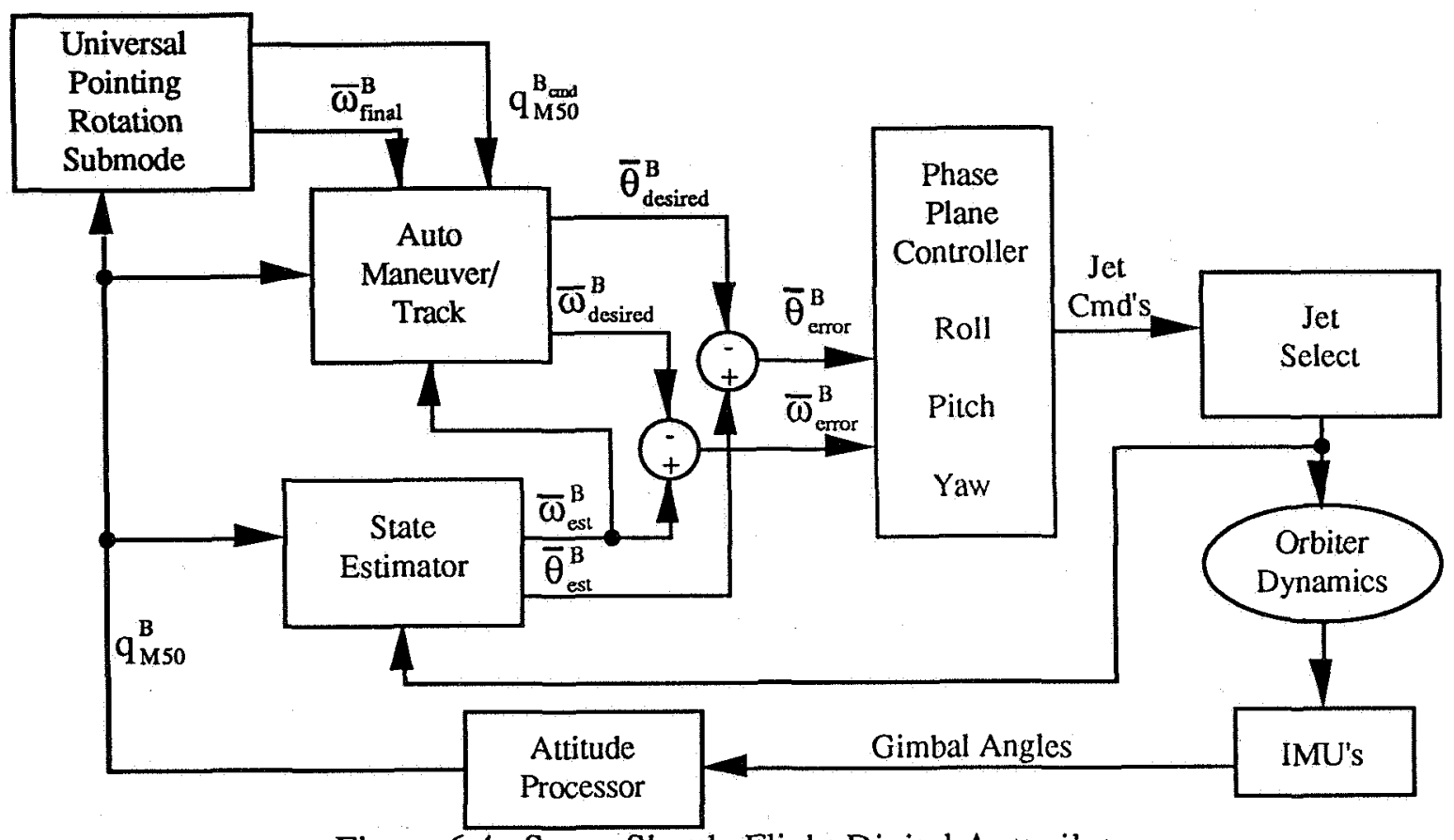

Figure 6.4. Space Shuttle Flight Digital Autopilot

\subsubsection{Universal Pointing Module}

The universal pointing module starts a prescribed attitude maneuver at a specified time.

This is accomplished by outputting to the auto maneuver module the desired final body attitude with respect to $\mathrm{M} 50, \mathrm{q}_{\mathrm{M} 50}^{\mathrm{B}_{\mathrm{cms}}}$, and the desired final body rate, $\bar{\omega}_{\text {final }}^{\mathrm{B}}$.

\subsubsection{State Estimator}

The state estimator takes as input the attitude measurement and the predicted rate change due to jet firings and outputs an estimate of the body rates and the body attitude. In the presence of flexible dynamics the rate estimate may be as much as 180 degrees out of phase. If the flexible modes are large enough to cause the rate error to exceed the rate limit 
(section 6.5.4) the poor phase information will cause firings in the wrong direction, further exciting the flexible modes and driving the FCS unstable. To prevent this, notch filters are employed on the attitude measurement. The notch filters attenuate attitude change at the frequency of the flexible modes, preventing the rate estimate from being driven by the flexible modes. Consequently, the rate error does not exceed the rate limit because of flexible dynamics, and stability is maintained. However, filtering of attitude measurements in this way does not prevent excitation of the bending dynamics.

For the space shuttle-GRO system two 2 nd order notches are used. They are centered on the first two modes. The notch filter width is taken to be $40 \%$ of the modal frequency for each mode.

\subsubsection{Auto Maneuver Module}

The auto maneuver module outputs desired body rate and attitude. If the attitude error, found by comparing $q_{M 50}^{B}$, the current attitude, with $q_{M 50}^{B_{c m s}}$, is larger than twice the deadband in any axis plus a bias angle representing the attitude excursion for buildup to the desired rate, then a maneuver at the specified maneuver rate, usually on the order of .2 $\mathrm{deg} / \mathrm{s}$, is commenced. This entails the following. An eigenaxis from the current to the specified attitude is computed. Rates in each body axis are determined, $\bar{\omega}_{\text {desired }}^{\mathrm{B}}$, which will achieve the maneuver rate about the eigen axis. "Lead" angles for each axis are determined which account for the angle traversed during the rate change. Lastly, the origins of the phase planes are shifted by $\bar{\omega}_{\text {desired }}^{\mathrm{B}}$ and the lead angles. During the maneuver, $\bar{\theta}_{\text {desired }}^{\mathrm{B}}$ is incremented according to the maneuver rate until the desired attitude minus the lead angle to decelerate is achieved. At this point $\bar{\omega}_{\text {desired }}^{\mathrm{B}}$ is set back to $\bar{\omega}_{\text {final }}^{\mathrm{B}}$ which, in the case of inertial attitude hold, is zero. 


\subsubsection{Phase Plane Modules}

Each axis is controlled by a separate phase plane which issues rotation commands for that axis. The vertical axes of the phase planes are the body rate error, $\bar{\omega}_{\text {error }}^{\mathrm{B}}$, and the horizontal axes are the body attitude error, $\bar{\theta}_{\text {error }}^{\mathrm{B}}$. Figure 6.5 shows a simple phase plane with one switch curve. Above the switch curve negative rate change is commanded and below it positive rate change is commanded. The goal is to achieve the origin where both the rate and attitude error will be zero.

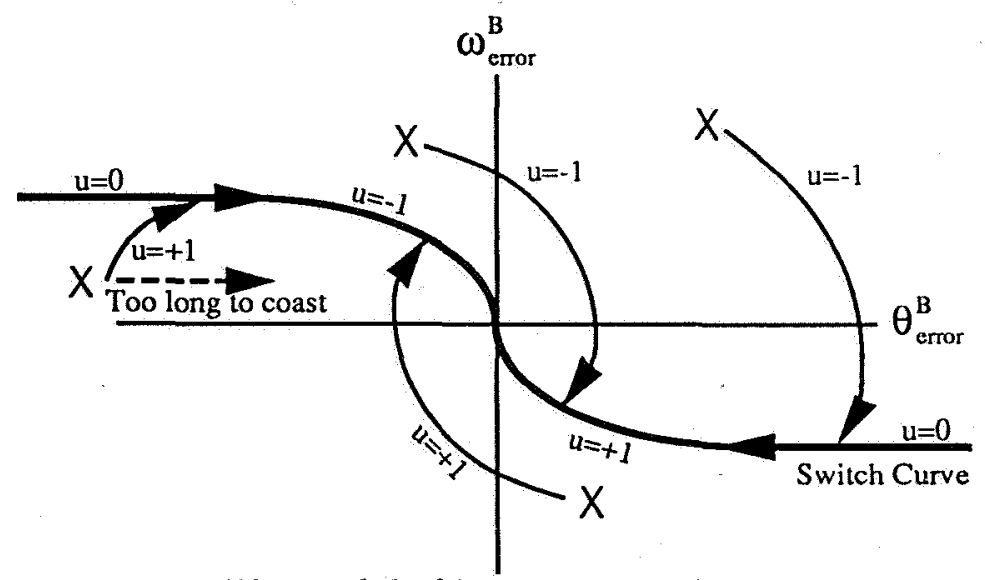

Figure 6.5. Simple Phase Plane

The problem with this phase plane is that it will continually command rate changes since the origin can never be reached exactly, due to measurement errors, disturbances, and rate change quantization. The solution as shown in Figure 6.6 is to place a deadband around the origin within which no rate change commands will be issued. This eliminates the constantly commanded rate change; however, it leads to high rate limit cycles. 


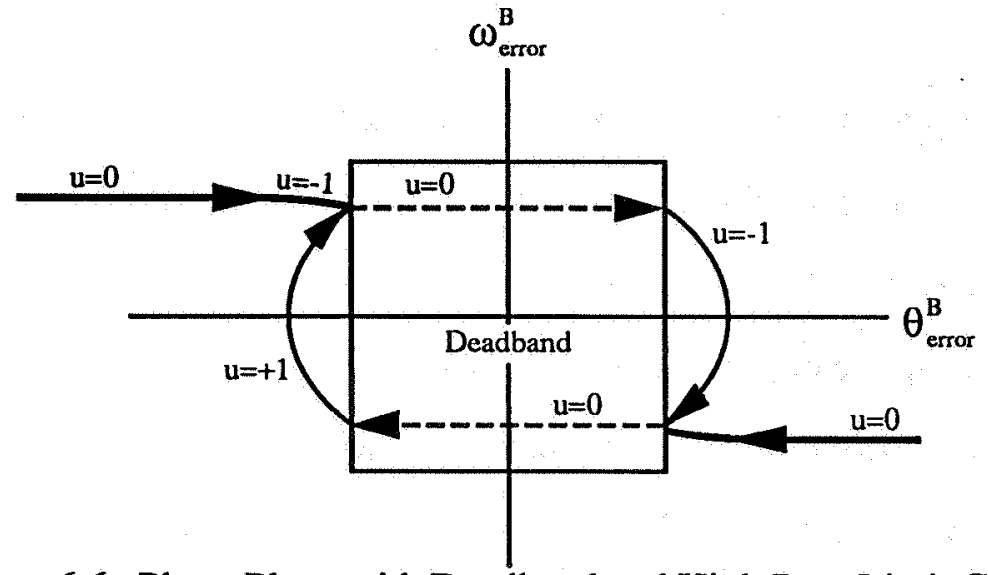

Figure 6.6. Phase Plane with Deadband and High Rate Limit Cycle

The cause of this problem is that the vertical switch curves delineating the deadband do not account for the change in attitude error which will occur while the rate error is being driven to zero. If these switch curves are set according to Eq. (6.2)

$$
\text { Switch Curve }\left(\omega_{\text {error }}^{\mathrm{B}}\right)= \pm \frac{\left(\omega_{\text {error }}^{\mathrm{B}}\right)^{2}}{2 \alpha_{\text {jet }_{\text {ererge }}}} \pm \text { Deadband }
$$

then the slowest possible limit cycle will be achieved (Figure 6.7), Functional Subsystem Software Requirements (FSSR)[18]. The rate of this limit cycle will be a function of the smallest rate change available also called the minimum impulse.

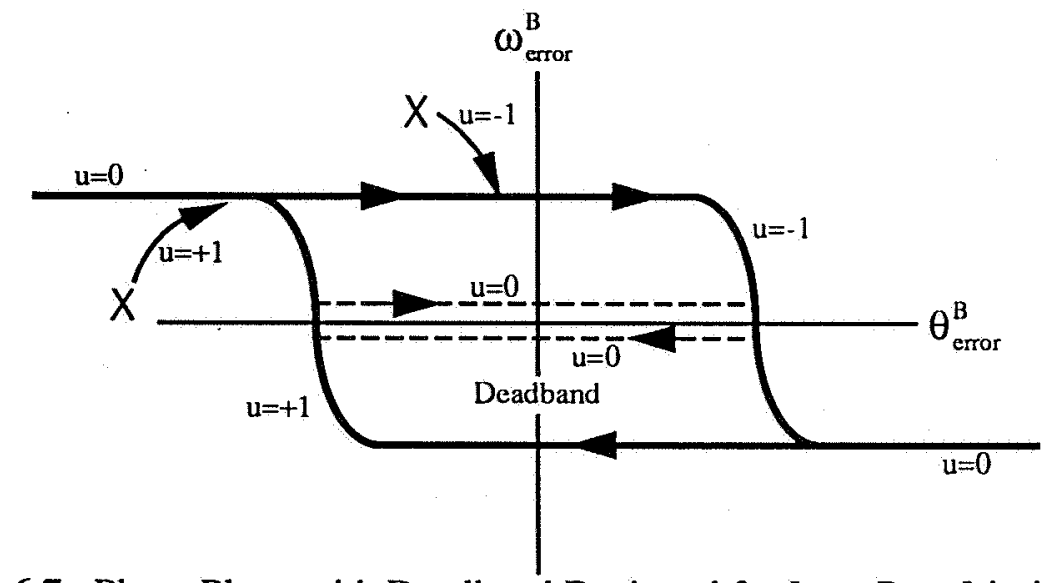

Figure 6.7. Phase Plane with Deadband Designed for Low Rate Limit Cycles 
The actual phase planes used on the space shuttle contain a few more features (Figure 6.8). Drift channels with a width of four tenths the rate limit were added to allow large attitude errors to diminish without commanding firings. Benches were added, typically one fifth of the deadband, to capture errant trajectories, preventing higher rate limit cycles. These errors may originate from achieving less than predicted acceleration, measurement transport delays, or quantization effects in the measurement. A biased switch curve, S11, based upon Eq (6.3) which uses the disturbance acceleration estimate, was added to set up one sided limit cycles in the presence of disturbances.

$$
\operatorname{Si1}\left(\theta_{\text {error }}^{B}\right)=-\sqrt{2\left|a_{d}\right|\left(\theta_{\text {error }}^{B}+.5 \text { Deadband }\right)}
$$

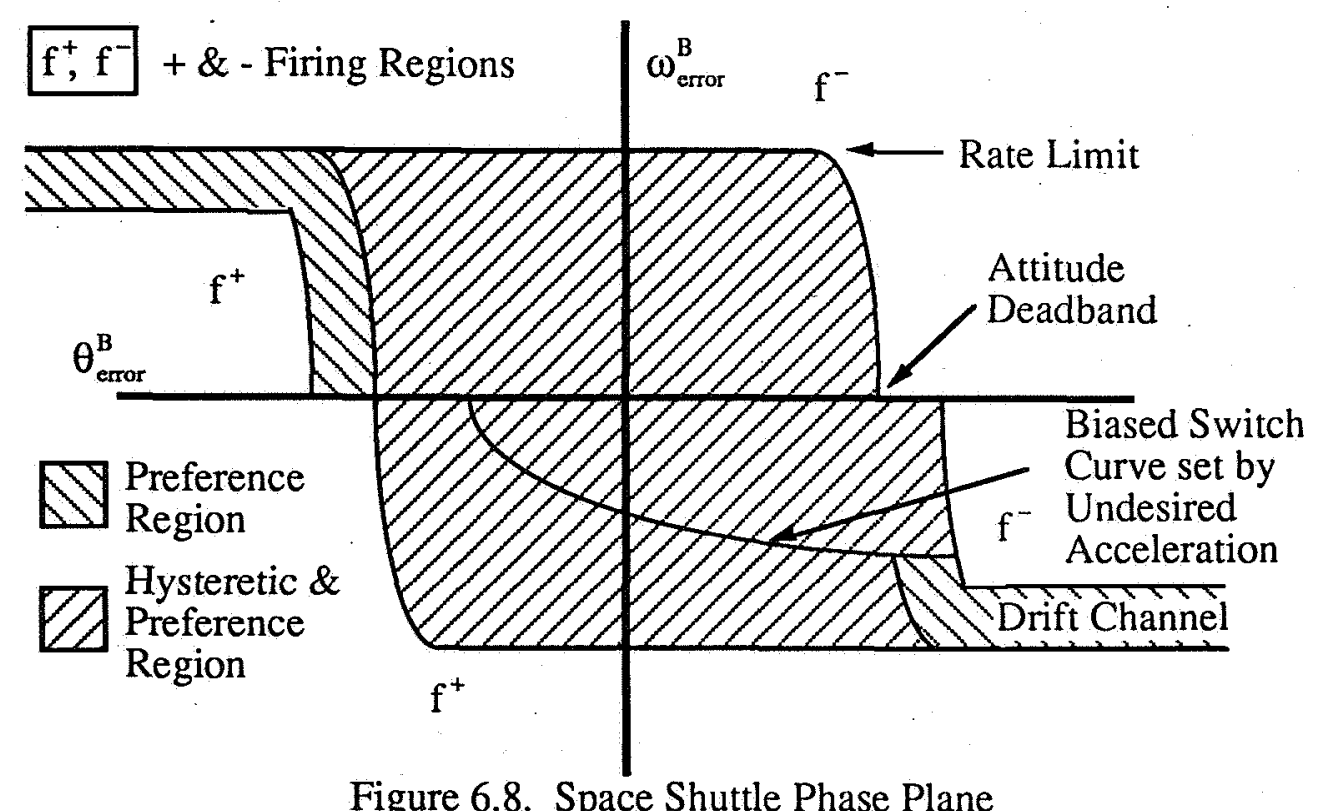

The output from the phase planes is a vector called rot_jet_cmd. The components of rot_jet_cmd are \pm 1 for axes whose phase planes are commanding a firing. The other components of rot_jet_cmd are set equal to the ratio of the rate error to the rate limit. The 
phase planes will command a firing while in the firing regions shown in Figure 6.8 or if the hysteretic region has just been entered, firings will continue to be commanded until the disturbance acceleration switch curve has been reached. For a complete specification of the space shuttle phase planes consult the FSSR document.

\subsubsection{Jet Select Module}

The jet select module operates every $80 \mathrm{msec}$, selecting jets for firing if $\mid$ rot_jet_cmdl is $\geq 1$ in any axis. If rot_jet_cmd has not been changed from the previous cycle the same jets will be fired again to help reduce jet duty cycles. A new jet selection is forced after five repetitions. Since a jet selection is performed every $80 \mathrm{msec}$ the criterion for selection is to maximize the amount of acceleration in the direction of rot_jet_cmd. Any excess rate change will always be approximately equal to or less than the minimum impulse, eliminating the need to consider the magnitude of rot_jet_cmd.

The jet selection is accomplished by taking the dot product of rot_jet_cmd with precomputed angular rate increments for each jet (The angular rate increments are the predicted amount that each jet will change the space shuttle body rates when fired for $80 \mathrm{msec}$ ). Specifically, it takes the dot product of each individual jet's angular increment with the rot_jet_cmd and ranks them from maximum dot product to minimum dot product. The first jet is selected for firing. If the second and third jets achieve acceleration beyond some threshold they too will be selected for firing, Hattis[15]. For Alt Mode, described below, jet selection may be limited to less than three jets by setting of the parameter, MAXJETS. This dot product scheme does not consider how much erroneous rate change is caused perpendicular to rot_jet_cmd. 


\subsubsection{Alt Mode}

The vernier jets are always selected according to the process described above; however, the primary jets can be operated in one of two modes. The original mode of operation uses a table lookup scheme to decide which jets to fire based upon the requested rate changes. A recently added mode of operation is Alt Mode. Jets are selected for Alt mode using the same algorithm as for verniers. Alt Mode allows the maximum jet on time to be specified as well as the minimum amount of time between jet firings. These parameters can be configured to reduce loads upon the space shuttle remote manipulator system while deploying payloads. For this thesis primaries will be run in Alt Mode without any firing restrictions.

\subsubsection{Modifications to Space Shuttle Flight Control System}

For this thesis the rate estimator shown in figure 6.4 was replaced with actual rate information to make fair comparisons between the fuzzy attitude controller, which uses actual rate, and the space shuttle FCS. 


\section{Chapter 7}

\section{Results}

In order to evaluate the fuzzy attitude controller, test runs were conducted on the Interactive On-orbit Simulation (IOS), a high fidelity simulation of the space shuttle, which can model all the reaction control system (RCS) autopilot capabilities of the space shuttle with and without man-in-the-loop. IOS was configured both to simulate the current space shuttle flight control system (FCS) as described in Chapter 6 and to use the fuzzy attitude controller presented in Chapter 4. For the fuzzy simulations, the auto-maneuver, phase plane, and jet select modules of the FCS were replaced with the fuzzy controller. In both cases actual rate information was used rather than rate estimates based upon IMU measurements to ensure fair comparisons between the two.

To evaluate the ease of adapting the fuzzy attitude controller to operate on different spacecraft, four maneuvers followed by attitude holds were conducted with two different mass property configurations and two different actuator setups. The first mass configuration was based upon a space shuttle alone, the second upon a space shuttle with GRO deployed on the SRMS at full extension above the payload bay. The GRO mass property cases included the combined orbiter-GRO system modeled as rigid; and, in order to evaluate the anti-flex rule package, modeled as flexible. The two sets of actuators used were the vernier jets and the primary jets operating in Alt Mode. The pointing 
specifications were equal to those normally used by the space shuttle while on-orbit. In moving from case to case the only changes to the fuzzy attitude controller were to the configurable parameters. The space shuttle flight control system automatically reconfigures analogous parameters based upon the mass properties.

Performance robustness to pointing requirements was characterized by conducting additional simulations with pointing specifications tightened beyond typical on-orbit levels. Runs were also conducted with errors in the flexible model of the orbiter-GRO system to evaluate anti-flex robustness to modeling error.

\subsection{Filter Designs}

This section describes the specific filter designs that were required by the fuzzy attitude controller for the simulated runs on IOS. They are based upon the general designs presented in Chapter 5.

\subsubsection{Slow Disturbance Estimator}

Two different filter designs were required for estimation of the low frequency accelerations acting on the space shuttle. The first one was used for the rigid body runs. The second one, with a much lower bandwidth, was used for the flexible body runs.

\subsubsection{Rigid Body Case}

For the rigid body runs the filter described by Eq. (5.1) was used. A rolloff frequency one decade before the control system sample frequency, $1 / \Delta \mathrm{t}$, or $12.5 \mathrm{~Hz}$ was chosen for the filter to attenuate errors in the predicted rate change from a jet firing. This dictates setting the pole at $.2(\mathrm{rad} / \mathrm{s})$. According to Eq. (5.6), with $\mathrm{T}=\Delta \mathrm{t}$, the discrete filter gain $\mathrm{K}$ should be set equal to .016 .

The control system sample frequency is actually the upper bound on the frequency content 
of the predicted rate change error. If a jet is fired for multiple cycles and it's angular rate increment is inaccurate, this error will be passed through the filter and affect the slow disturbance estimate. The estimate will also be degraded if the error in acceleration caused by the inaccurate angular rate increments is of the same or greater magnitude than the disturbance acceleration, since in the most optimistic case the error is only being attenuated by an order of magnitude. These issues did not pose a problem for this thesis because predicted rate change error was small.

\subsubsection{Flexible Body Case}

According to table 6.2, the disturbances due to bending of the SRMS with GRO need attenuation by approximately three orders of magnitude to prevent masking the low frequency disturbance accelerations from gravity gradient and aerodynamic loading. The first order filter of Eq. (5.1) proves inadequate for this task as the required rolloff frequency would be at least three decades before the first flexible mode in table 6.3. The resultant time constant of the filter would be greater than the time required for the space shuttle to orbit the earth $(5400 \mathrm{sec})$, preventing any accurate estimate from being achieved to support efficient attitude hold. A higher order filter is required. Eq. (7.1) shows the modified disturbance estimator which first passes the residual acceleration through a high order filter as presented in Chapter 5.

$$
\begin{aligned}
\bar{x}_{n+1}^{\text {filt }} & =A_{\text {filt }} \bar{x}_{n}^{\text {filt }}+B_{\text {filt }}\left[\frac{\bar{\omega}_{n}^{B}-\left(\bar{\omega}_{n-1}^{B}+\Delta \bar{\omega}_{\text {jet }_{n}}\right)}{T}\right] \\
y_{n} & =C_{\text {filt }} \bar{x}_{n}^{\text {filt }} \\
\bar{a}_{d n} & =\bar{a}_{d n-1}-K\left(\bar{a}_{d n-1}-y_{n}\right)
\end{aligned}
$$

In addition to attenuating the first mode of the flexible dynamics by three orders of magnitude, the filter transients should be fast. If the transients last as long as a typical limit 
cycle, then the disturbance acceleration estimate will still be corrupted when the rate change for the slow disturbance trajectory is calculated, causing an inappropriate trajectory.

Both Butterworth and Chebyshev filters were evaluated as candidates for the filter in Eq. (7.1). The Butterworth pole pattern was chosen because it demonstrated faster transients than Chebyshev. In order to evaluate what order Butterworth filter would provide the best performance, Figure 7.1 was generated. It shows the settling time for different order Butterworth filters, where each order of filter is adjusted to attain three orders of magnitude attenuation for a range of frequencies. The settling time was defined as the time required for the transient to a unit sinusoid, input to the filter at the given frequency, to diminish below .01 .

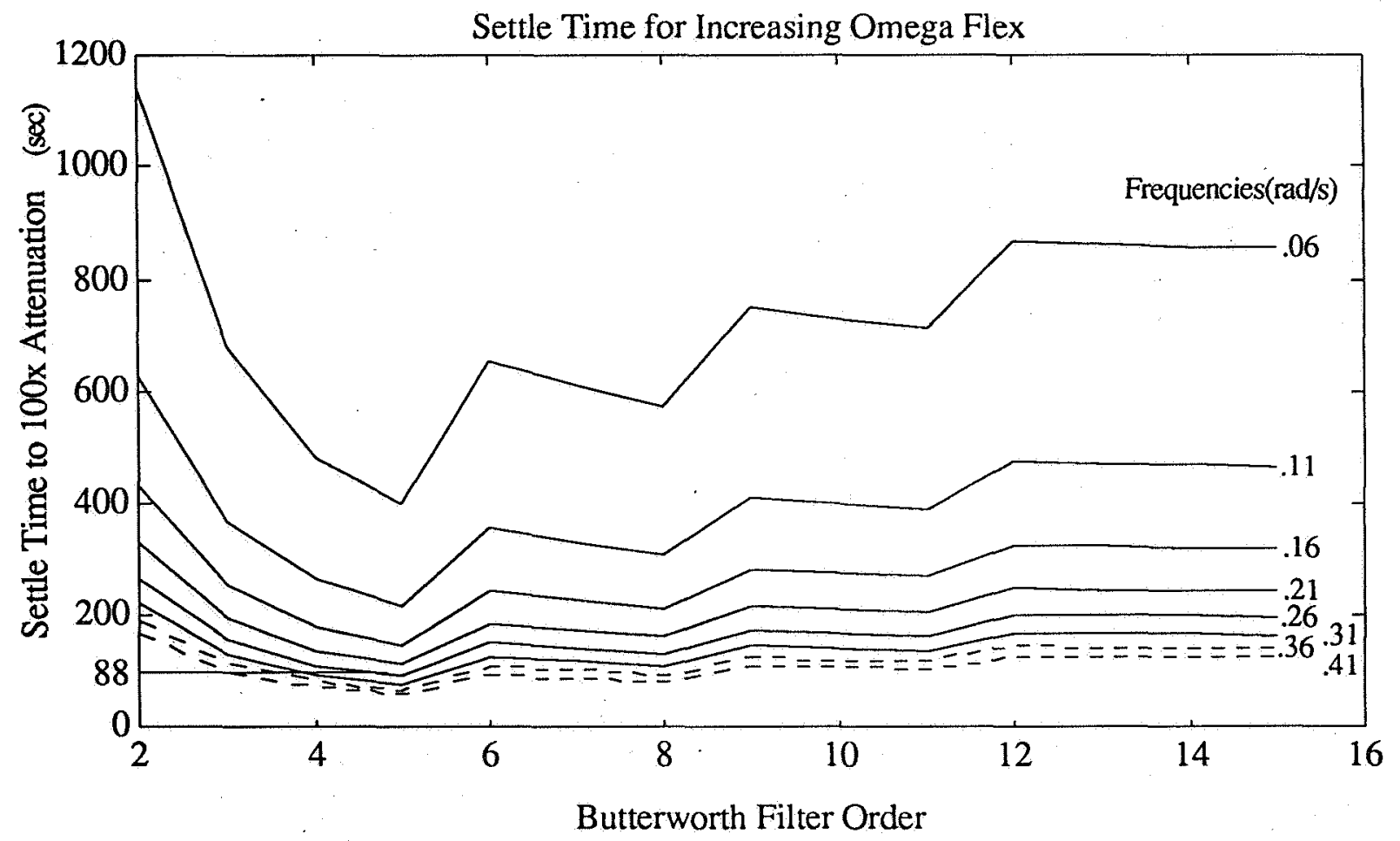

Figure 7.1. Settling Times vs. Butterworth Filter Order for Various Input Frequencies

Figure 7.2 shows the maximum transient peak to unit sinusoidal inputs at the frequencies shown in Figure 7.1. The peak, independent of the input sinusoidal frequency, is only a . 
function of filter order.

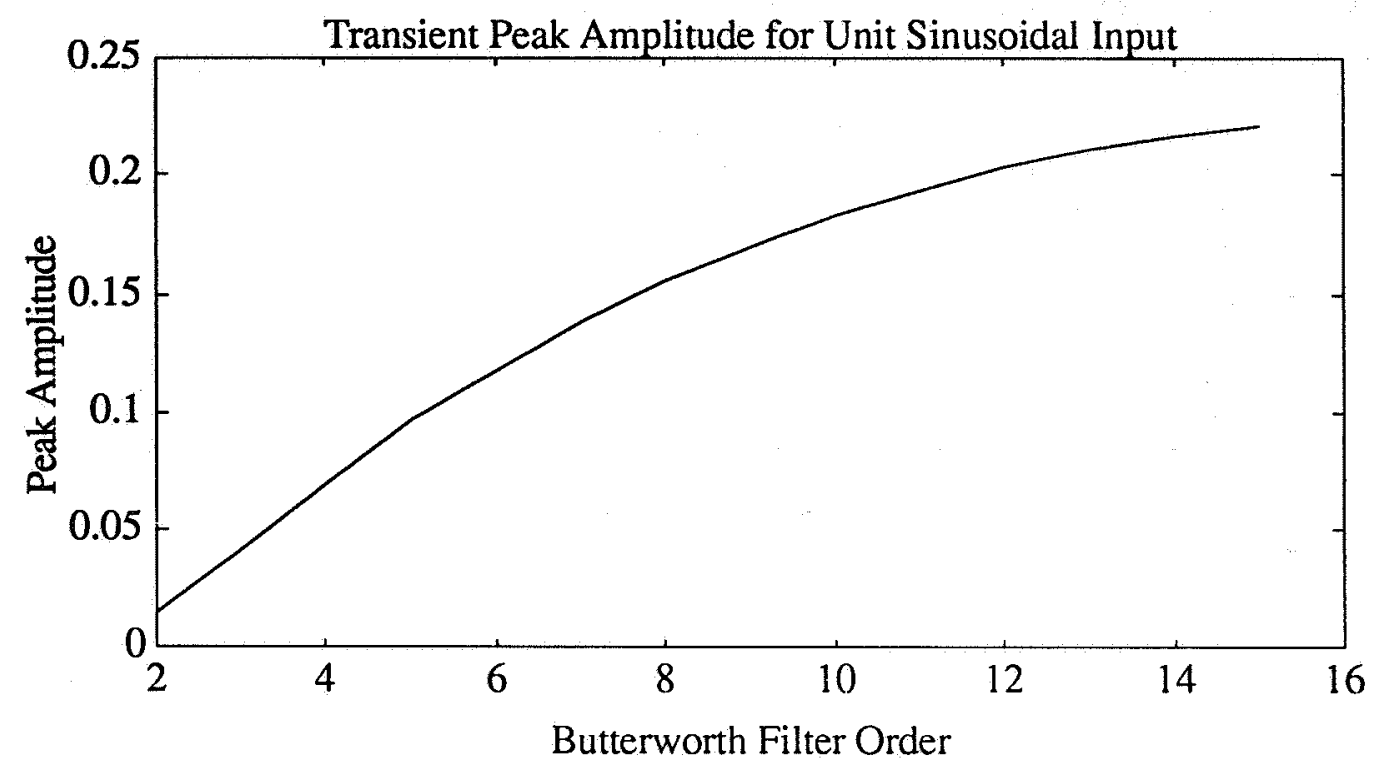

Figure 7.2. Peak Transient Amplitudes vs. Butterworth Filter Order

The fifth order Butterworth filter was chosen for its fast settling time of 88 seconds to the frequency of the first flexible mode of the orbiter-GRO system. However, with a peak transient of .08 , slow disturbance estimates during the transient period will not contain acceptable slow disturbance acceleration estimates. To prevent a poor slow disturbance trajectory from being initiated, if the time since the last jet firing is less than the 88 second settling time then $\Delta \bar{\omega}_{\text {cmd }_{\text {slow }}}$ will be set equal to the rate change requested by the baseline rule package rather than the slow disturbance rules. This will not happen usually since most limit cycle times will be greater than the settling time.

The required rolloff frequency to attain three orders of magnitude attenuation at the first flexible mode can be determined using Eq. (7.2)

$$
\omega_{\text {rolloff }}=10^{\left(\log _{10} \omega_{\text {flex }}-3 / n\right)} ; n=\text { Filter Order }
$$


Figure 7.3 demonstrates that the desired level of attenuation at the first flexible mode is achieved while maintaining a constant unity gain in the pass band.

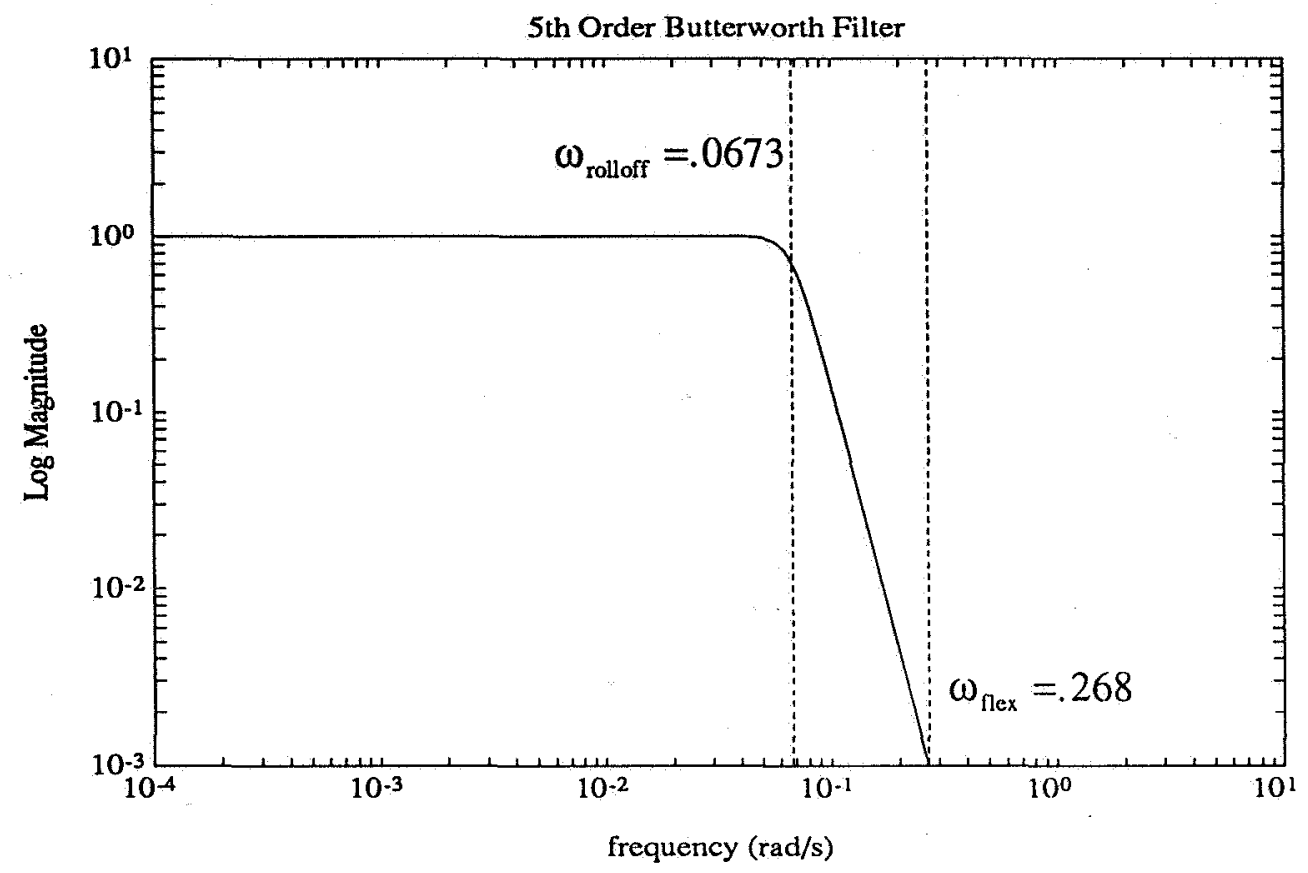

Figure 7.3. Frequency Response of 5th Order Butterworth Filter

The slow disturbance estimator including the 5th order Butterworth filter, Eq. (7.1), was evaluated by comparing its estimate during a simulated maneuver in IOS, including modeling of the flexible orbiter-GRO dynamics, to that from the slow disturbance estimator designed for the rigid cases during an identical simulated maneuver not modeling the flexible dynamics, which reflects the actual slow disturbance acceleration. The two time histories are overlaid in Figure 7.4.

Performance of the slow disturbance estimator in the presence of flexibility was better than expected. With the exception of the large transients during the first few hundred seconds due to the flexible modes being pumped by the large rate changes required for the . maneuver, the estimate contains useful information. Since the estimate is not used during the maneuver, this poses no problem. Transients due to subsequent attitude hold firings 
are much smaller and decay rapidly enough such that the estimate is accurate when it is time to calculate another slow disturbance trajectory rate change.

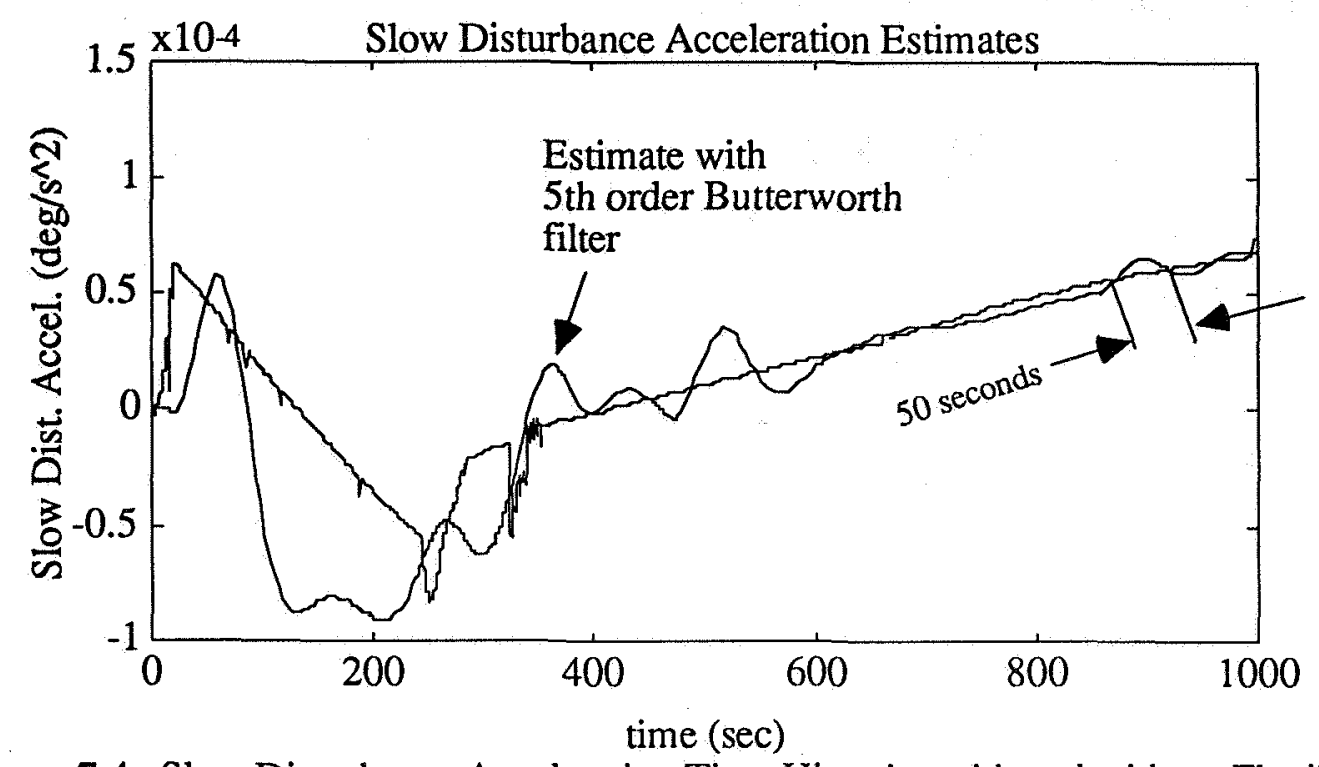

Figure 7.4. Slow Disturbance Acceleration Time Histories with and without Flexible Disturbances and Higher Order Filtering

\subsubsection{Rigid Rate Estimator}

When flexibility of the space shuttle-GRO system was included in the simulation dynamics, a model based filter with frequency weighting on the residual, $\mathrm{Eq}(5.19)$, was chosen to estimate the rigid and flexible components of the rate from the total rate measurement. This approach was chosen over a Kalman filter with frequency weighting on the measurement noise, and a model based filter without frequency weighting because of its ease of design and its superior performance .

The gain vector and the roll-off frequency of the 2 nd order filter were determined using a Simplex search method, where the cost function to be minimized was the RMS of the error between the environment rigid rate and the estimated rigid rate over time for several attitude maneuvers. For best performance, the filter was set to roll-off at $.0255(\mathrm{rad} / \mathrm{s})$, a little more than a decade before the first GRO mode at $.268(\mathrm{rad} / \mathrm{s})$, providing effective attenuation of 
the residual error at the frequencies due to flexible modes while passing errors in the residual at lower frequency. $H_{x}$ was set at $.0137, H_{z 1}$ at -.0738 , and $H_{z 2}$ at -2.6652 in Eq. (5.19). Figure 7.5 shows the frequency response of the frequency weighting filter.

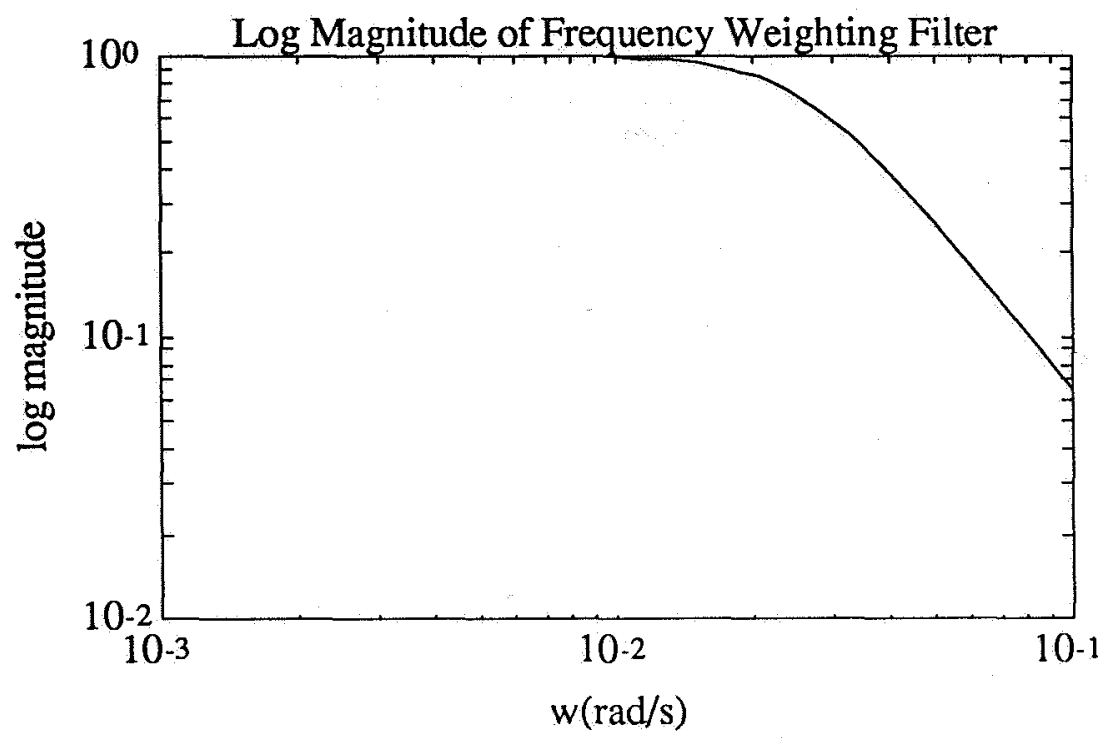

Figure 7.5. Frequency Response of Frequency Weighting Filter

A maneuver including flexibility was simulated to evaluate the rigid rate estimator. Figure 7.6 shows a plot of the actual total and rigid body rate for the roll axis. The flexible mode at $.316 \mathrm{rad} / \mathrm{s}$ is visible in the total body rate. Figure 7.7 shows the rigid rate from the same maneuver and estimates of the rigid rate with and without frequency weighting. The frequency weighted estimate has smaller steady state error indicative of higher gain, but still manages to reject a greater portion of the rate due to flexible modes. The important result is that the error between the rigid rate estimate with frequency weighting and the actual environment rigid rate, Figure 7.7 , is never greater than about $20 \%$ of the difference between the total rate and rigid rate in Figure 7.6. This means that the flex rate estimate will have sufficient directional accuracy to make the anti-flex rules useful. 


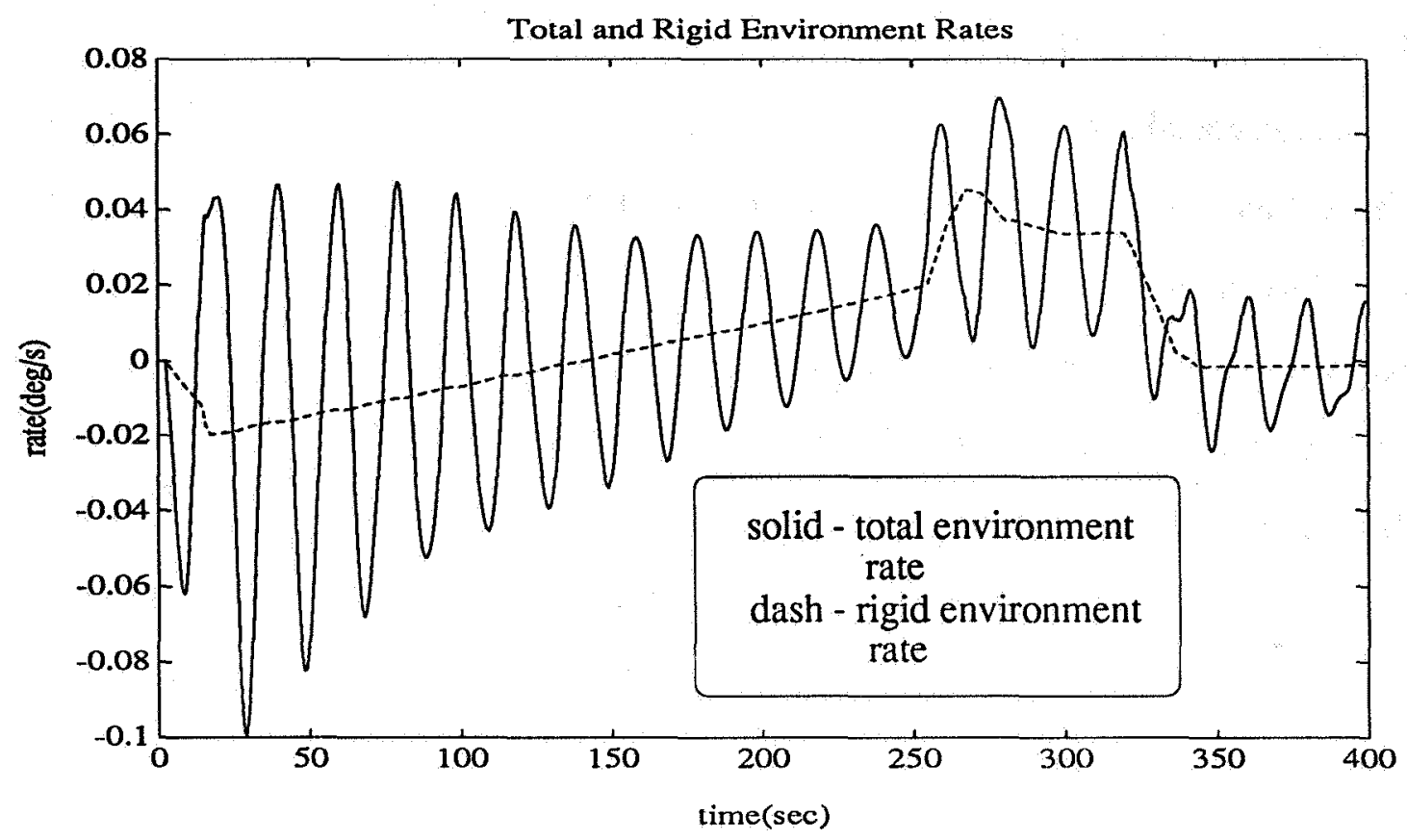

Figure 7.6. Total and Rigid Body Environment Rates

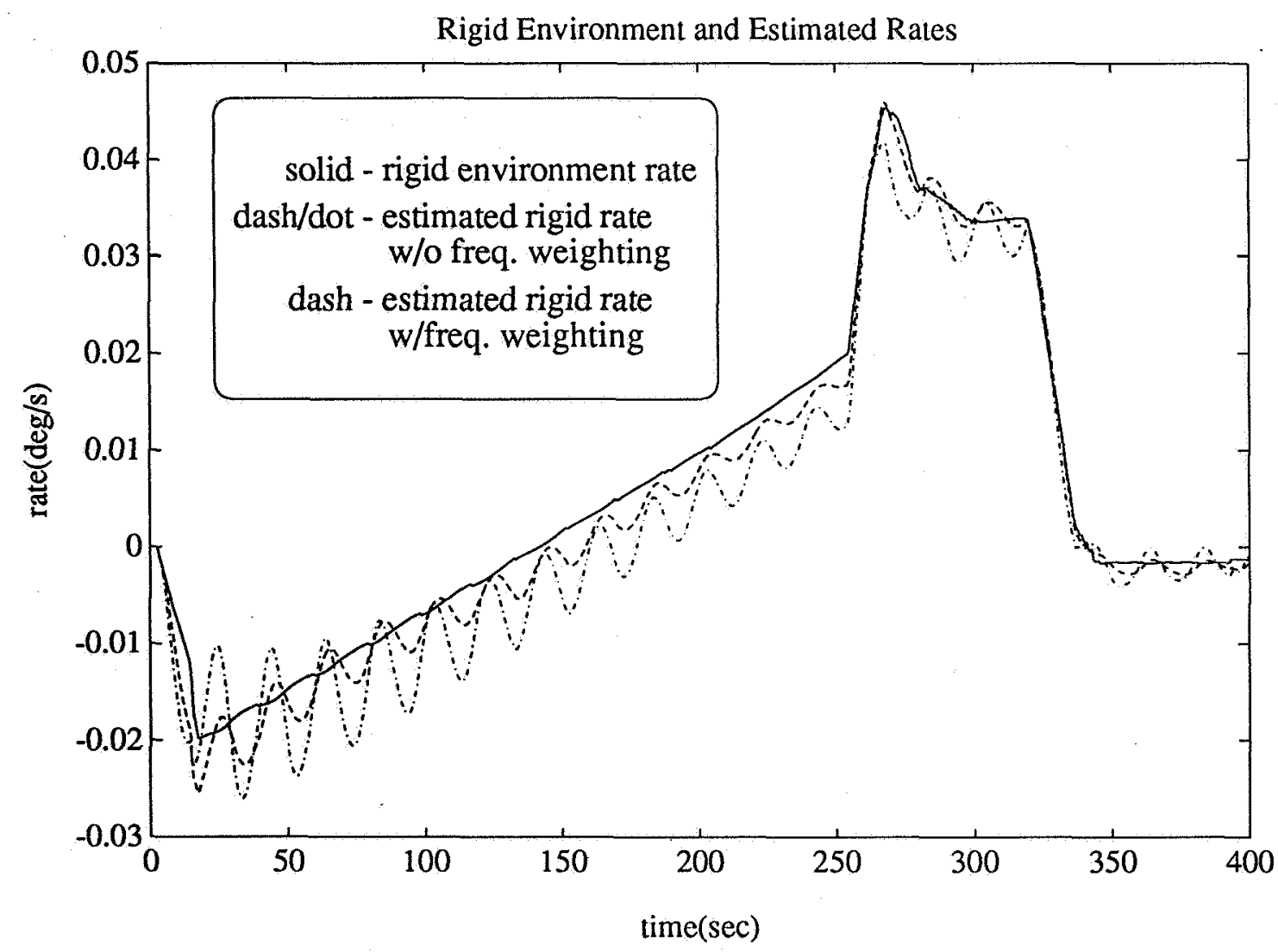

Figure 7.7. Actual and Estimated Rigid Rates with and without Frequency Weighting 


\subsection{Nominal Results}

This section contains results from runs conducted on IOS with parameters configured to reflect the normal envelope of operations for the space shuttle while on-orbit. In all cases except those modeling flexibility, simulation runs were 6000 seconds. For the flexibility cases runs were 2000 seconds due to computer resource constraints. Four different inertial attitude maneuvers were performed and are listed in table 7.1 (note that sequences are M50 to body rotations). Each maneuver was followed by an inertial attitude hold for the duration of the 6000 seconds, allowing evaluation of attitude hold performance over at least one 90 minute orbit, progressing through a full cycle of the slow disturbance accelerations. The maneuvers were chosen to require rates in all axes and similar amounts of total rotation in different directions.

Table 7.1. Maneuver Euler Angle Sequences

\begin{tabular}{|c|c|}
\hline Maneuver & $\begin{array}{c}\text { Euler Angle } \\
\text { Sequence } \\
\text { Pitch, Yaw, Roll }\end{array}$ \\
\hline A & $(45,30,-10)$ \\
\hline B & $(45,-30,20)$ \\
\hline C & $(-45,30,-15)$ \\
\hline D & $(30,-15,-45)$ \\
\hline
\end{tabular}

The input parameters for the space shuttle flight control system and their values are listed in table 7.2.

Table 7.2. Parameters for Space Shuttle Flight Control System

\begin{tabular}{||l|c|c|}
\hline \multicolumn{1}{|c|}{ Parameter } & Verniers & Alt Mode \\
\hline Deadband & $1(\mathrm{deg})$ & $3(\mathrm{deg})$ \\
\hline Rate Limit & $.02(\mathrm{deg} / \mathrm{s})$ & $.1(\mathrm{deg} / \mathrm{s})$ \\
\hline Maneuver Rate & $.2(\mathrm{deg} / \mathrm{s})$ & $.2(\mathrm{deg} / \mathrm{s})$ \\
\hline MAXJETS & 3 & 3 \\
\hline
\end{tabular}

The equivalent parameters for the fuzzy attitude controller are listed in table 7.3. The pointing error constraint differs from the deadband in that it refers to the maximum allowable eigenangle error rather than maximum attitude error allowed per axis. To permit 
the same maximum eigenangle error, the pointing error constraint should equal the square root of the sum of the squares of each axis' deadband or the square root of three times the deadband if the deadbands are all the same, as they are for the space shuttle. The pointing error constraints were actually set slightly smaller than this so that the resultant average eigenangle error from attitude holds for the fuzzy attitude controller and the FCS, which rarely has maximum error in all three axes, would be comparable.

Table 7.3. Parameters for Fuzzy Attitude Controller

\begin{tabular}{|l|c|c|}
\hline \multicolumn{1}{|c|}{ Parameter } & Verniers & Alt Mode \\
\hline Pointing Error Constraint & $1.5(\mathrm{deg})$ & $4.3(\mathrm{deg})$ \\
\hline Rate Error Constraint & $.02(\mathrm{deg} / \mathrm{s})$ & $.1(\mathrm{deg} / \mathrm{s})$ \\
\hline Maneuver Rate & $.2(\mathrm{deg} / \mathrm{s})$ & $.2(\mathrm{deg} / \mathrm{s})$ \\
\hline MAXJETS & 3 & 3 \\
\hline
\end{tabular}

Runs were conducted with various combinations of the rule packages active and labeled according to table 7.4. For example, 'sf', indicates that the slow disturbance and jet limiting rules were active. If 's' is not in the label it is understood that the baseline rules were active.

Table 7.4. IOS Run Label Notation

\begin{tabular}{|c|c|}
\hline Notation & Meaning \\
\hline Orig & Space Shuttle FCS \\
\hline Base & Baseline Rules Only \\
\hline s & $\begin{array}{c}\text { Slow Disturbance } \\
\text { Rules }\end{array}$ \\
\hline f & Jet Limiting Rules \\
\hline c & Anti-Chatter Rules \\
\hline m & Anti-Flex Rules \\
\hline
\end{tabular}

\subsubsection{Orbiter Alone}

Table 7.5 lists the configurable parameters used by the fuzzy attitude controller for the simulations using orbiter alone mass properties. The rule package weights were set by trial and error. After a couple simulation runs, the listed values were found to provide good results. The values certainly are not the optimum choices, but the point was not to iterate 
many times to find the best numbers but rather to quickly arrive at some values which work well for the given task. In Alt Mode the anti-chatter rule package was weighted less because the larger thrust from the primary jets led to burns with very few jet cycles. Inhibiting the controller's ability to turn jets on and off as much as when using verniers, which typically have many more jet cycles per burn, leads to poorer performance.

The values for average control acceleration were derived by looking at the typical rate changes a jet selection will cause. Care should be taken when selecting this value. It is used to size the fuzzy sets for big and small $\alpha$, Figure 4.4. It is also used to calculate $\theta_{\text {lead }}$, which in turn is used to size big and small $\theta$. For big and small $\alpha$, the value selected is not too critical as it is just characterizing the range of expected $\alpha$. While conducting maneuvers, the performance of the controller is much more sensitive to the value of $\theta_{\text {lead }}$. The primary problem lies in choosing the average control acceleration too large. The resulting small $\theta_{\text {lead }}$ causes overshoot when decelerating from a maneuver which wastes fuel and increases jet duty cycles. It is best to prevent this by choosing a conservative value for the average control acceleration.

Methods exist for choosing $\beta$, Figure 4.5 , such that the average pointing error will approach zero, Bergmann[11]; however, for these simulations it was found more effective as a means of preventing two sided limit cycles. $\beta$ determines what fraction of the error envelope's radius or pointing error constraint the slow trajectory should traverse beyond the error envelope's origin. Larger $\beta$ causes closer approaches to the maximum allowable error on the opposite side of the error envelope. Due to Alt Mode's large minimum impulse, $\beta$ was set equal to zero, since it is unlikely that the rate change required for a slow trajectory will be accurately achieved. This helps prevent the attitude error from excursing too far during the slow trajectory and causing unnecessary firings. $\beta$ was set larger for verniers since the rate change requested for the slow trajectory can be achieved with much greater accuracy. 
The minimum impulse determines how precisely the slow trajectory rate change will be performed, Figure 4.6. If it is set too big, the desired slow trajectory will not be achieved with accuracy. More importantly, if it is set too small, the controller will chatter the jets in an attempt to achieve the desired slow trajectory rate. It is best to err by setting the minimum impulse too large. The values listed in table 7.5 were found by starting with a value which was too small and increasing it until the controller no longer chattered when initiating a slow trajectory.

Table 7.5. Configurable Parameters for Fuzzy Attitude Controller, Orbiter Alone Cases

\begin{tabular}{|c|c|c|}
\hline Parameter & Verniers & Alt. Mode \\
\hline Jet Limiting Package Weight & .2 & .2 \\
\hline $\begin{array}{c}\text { Anti-Chatter Package } \\
\text { Weight }\end{array}$ & .3 & .1 \\
\hline Anti-Flex Package Weight & 0 & 0 \\
\hline $\begin{array}{c}\text { Average Control } \\
\left.\text { Acceleration.(deg/s }{ }^{2}\right)\end{array}$ & .005 & .8 \\
\hline Minimum Impulse(deg/s) & .003 & .045 \\
\hline$\beta$ for slow trajectory & .5 & 0 \\
\hline
\end{tabular}

\subsubsection{Maneuver Results}

There are several criteria which should be measured to quantify the performance of an attitude controller. In performing maneuvers the amount of fuel used is important because often this dictates the duration for which the spacecraft will function usefully. Jet duty cycles are also important, especially for reusable spacecraft such as the space shuttle. Duty cycles dictate how often the thrusters will require replacement. Another important factor is maneuver completion time. Fair comparisons of fuel usage for different controllers require that maneuvers should be completed in a similar amount of time since a significant amount of fuel is used to accelerate and decelerate from the desired maneuver rate. First, results for the four maneuvers with verniers are presented followed by Alt Mode results. 
Figure 7.8 shows the amount of fuel used and the number of jet switches required to perform each of the four maneuvers for the FCS and the fuzzy attitude controller using verniers with various combinations of rule packages active. Jet switches should not be confused with firings. For every firing, a jet must switch on and then off. Thus, to find the number of firings, divide the number of switches by two.

In general, the FCS out-performed the fuzzy attitude controller as can be seen by the averages in Figure 7.9. It used slightly less fuel and required fewer jet switches; however, this is the region of operation within which the space shuttle flight control system performs best. It has been repeatedly tuned for over 15 years to arrive at this point.

The results demonstrate the effect of the jet limiting rule package and the anti-chatter rule package as compared to the baseline or slow disturbance rule packages operating alone. The jet limiting package tends to aid fuel economy. For every run, when the jet limiting package was added, fuel consumption was less than for the previous run without the package. The anti-chatter rule package reduces the number of jet switches in every case by a factor of two. It is worth noting that the combination of the two, the ' $\mathrm{fc}$ ' and 'sfc' runs, does not reduce gains achieved by either rule package individually. 


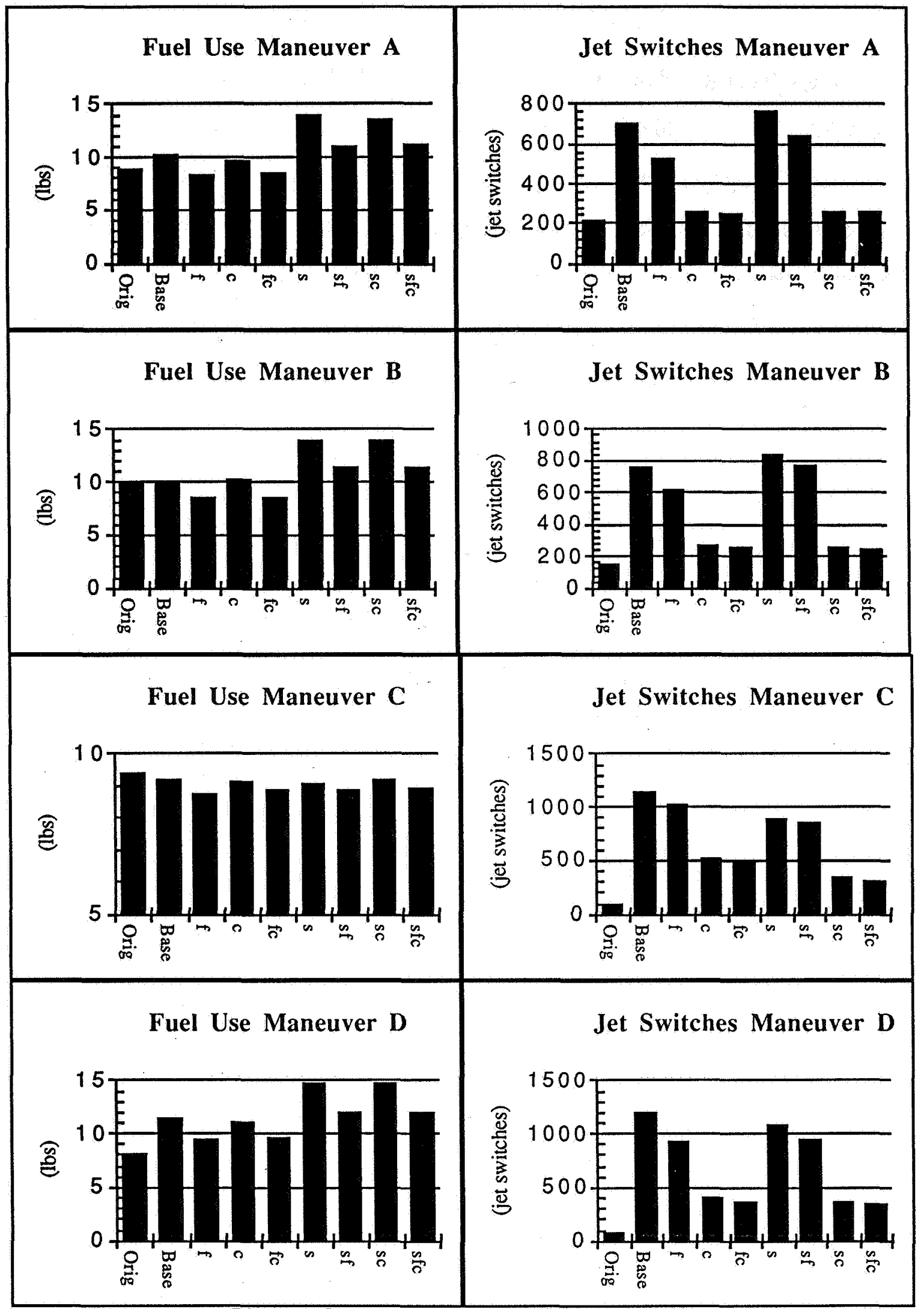

Figure 7.8. Maneuver Results for Vernier Jets 
The average fuel use and number of jet switches over the four maneuvers is presented in Figure 7.9. The runs which used the slow trajectory rule package instead of the baseline rules used slightly more fuel. This is due to the transition that occurs in the rules as the eigenangle becomes smaller than the pointing error constraint. At this point the objectives change and jets are selected according to the slow trajectory command. This leads to slightly worse fuel performance for maneuvers but the benefits in reduced jet cycles during attitude hold are apparent in the following section.

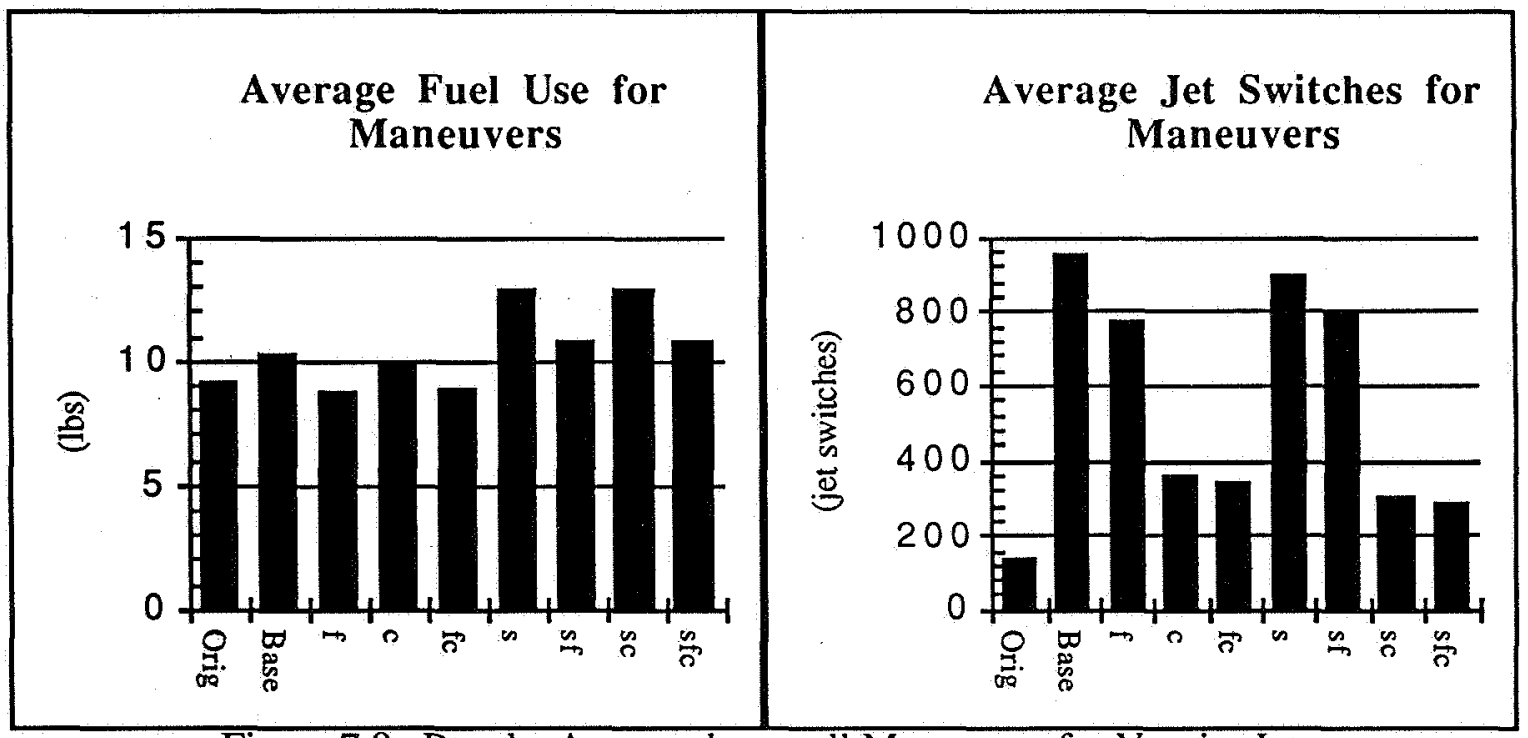

Figure 7.9. Results Averaged over all Maneuvers for Vernier Jets

On average the runs including the slow trajectory package also completed the maneuvers more slowly as shown in Figure 7.10, again due to the transition in priorities as the eigenangle becomes smaller than the pointing error constraint. The other maneuver times are comparable with the FCS allowing fair comparison of fuel consumption (longer maneuver times indicate a slower maneuver rate which means less rate change at the beginning and end of a maneuver ultimately requiring less fuel). 


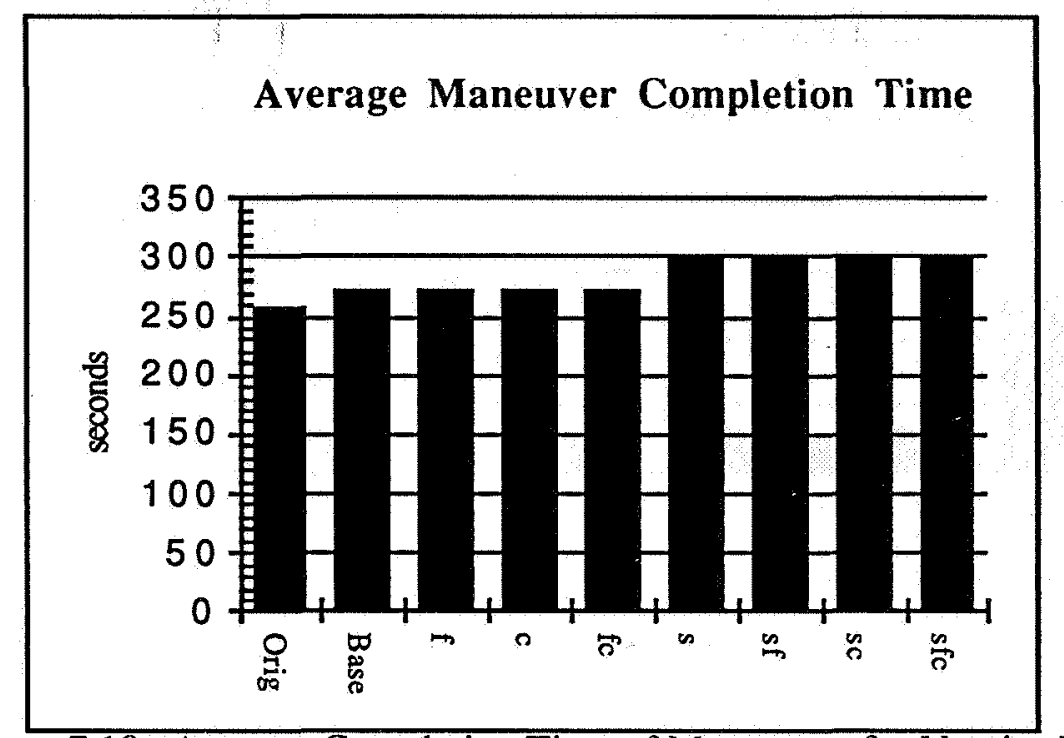

Figure 7.10. Average Completion Time of Maneuvers for Vernier Jets

Figures $7.11,7.12$, and 7.13 show the results for the same maneuvers using the primary jets in Alt Mode. The fuzzy attitude controller out-performs the FCS in all respects, using less fuel, fewer jet switches, and in some cases, less time to complete the maneuvers. There are a few notable differences from verniers when using the primary jets in Alt Mode.

The first difference is the unsuitability of the baseline rule package for this actuator system. The baseline rule package contains no means for explicitly stating the dimensions of an attitude error hysteresis region. It only allows specification of how much error between the desired and the actual eigenangle rate is tolerable through the rate error constraint. Since the desired eigenangle rate is a function of the eigenangle, which reflects the total attitude error, this does provide a certain amount of attitude hysteresis. However, to make it large enough to prevent the primary jets from chattering continuously, the rate error constraint must be increased to unacceptable levels. The slow disturbance rule package specifies a region, through the pointing error constraint, within which the slow disturbance trajectory is followed. This provides the attitude error hysteresis region necessary for attitude control using Alt Mode. The baseline rules will not be used in Alt Mode. 


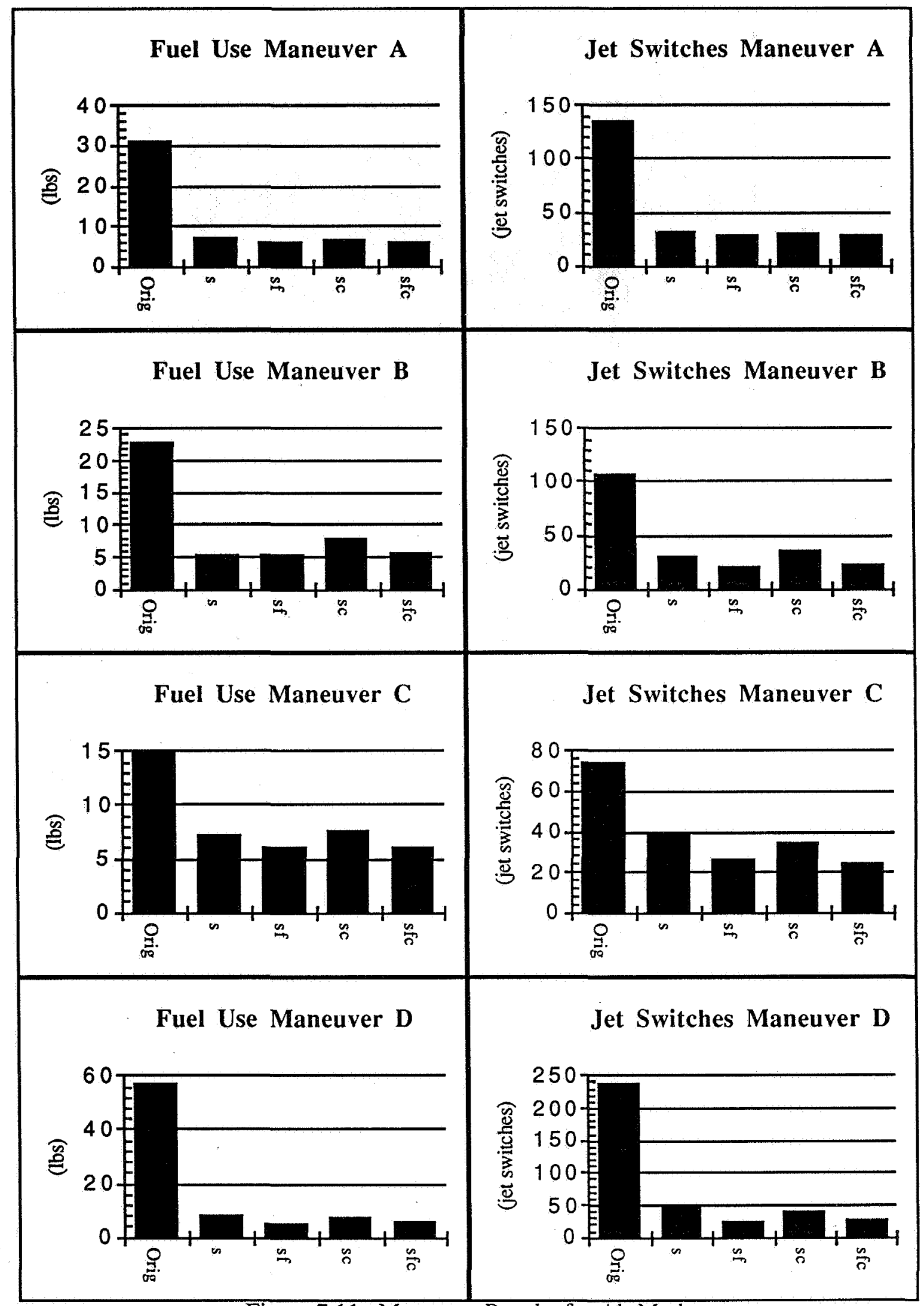

Figure 7.11. Maneuver Results for Alt Mode 


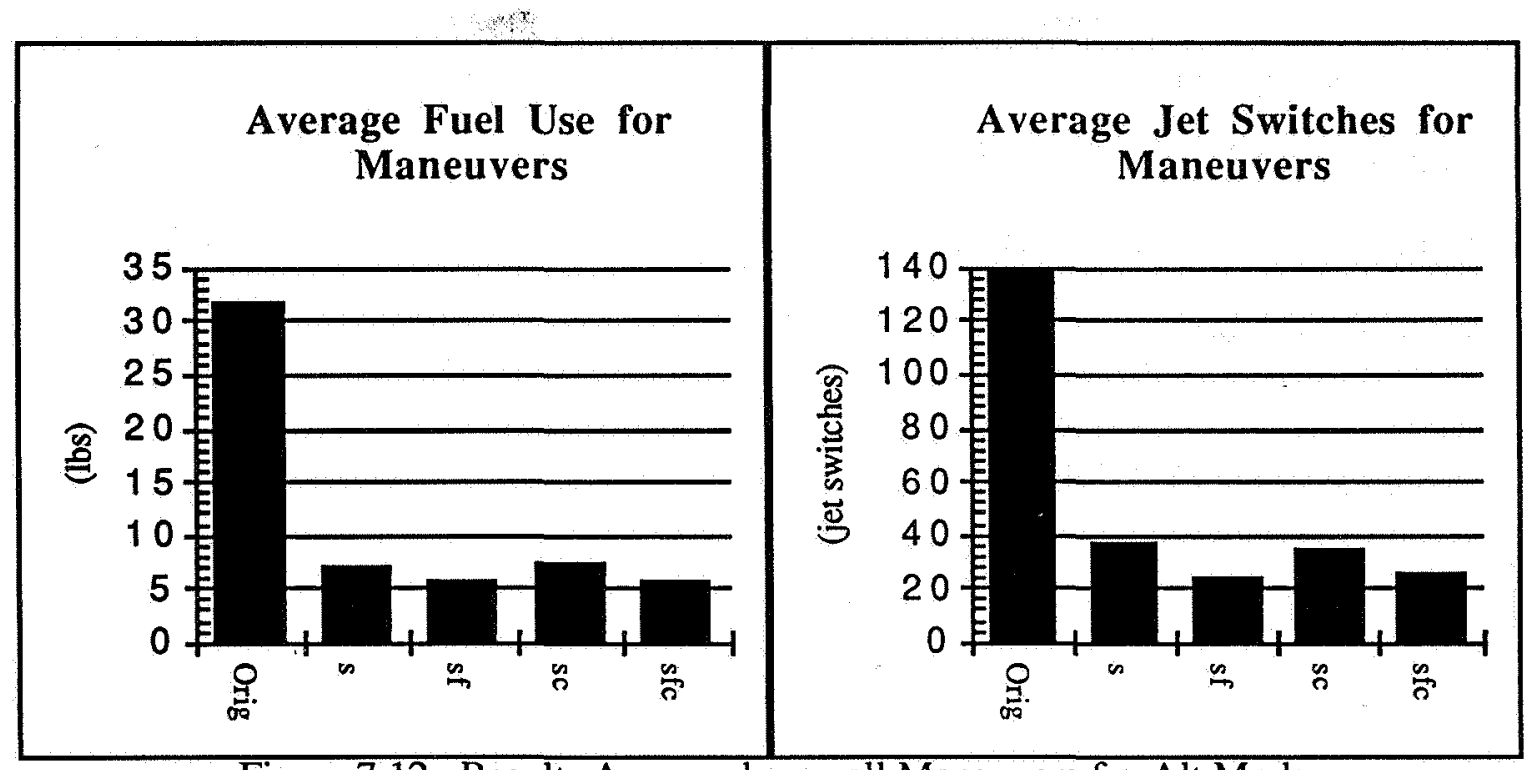

Figure 7.12. Results Averaged over all Maneuvers for Alt Mode

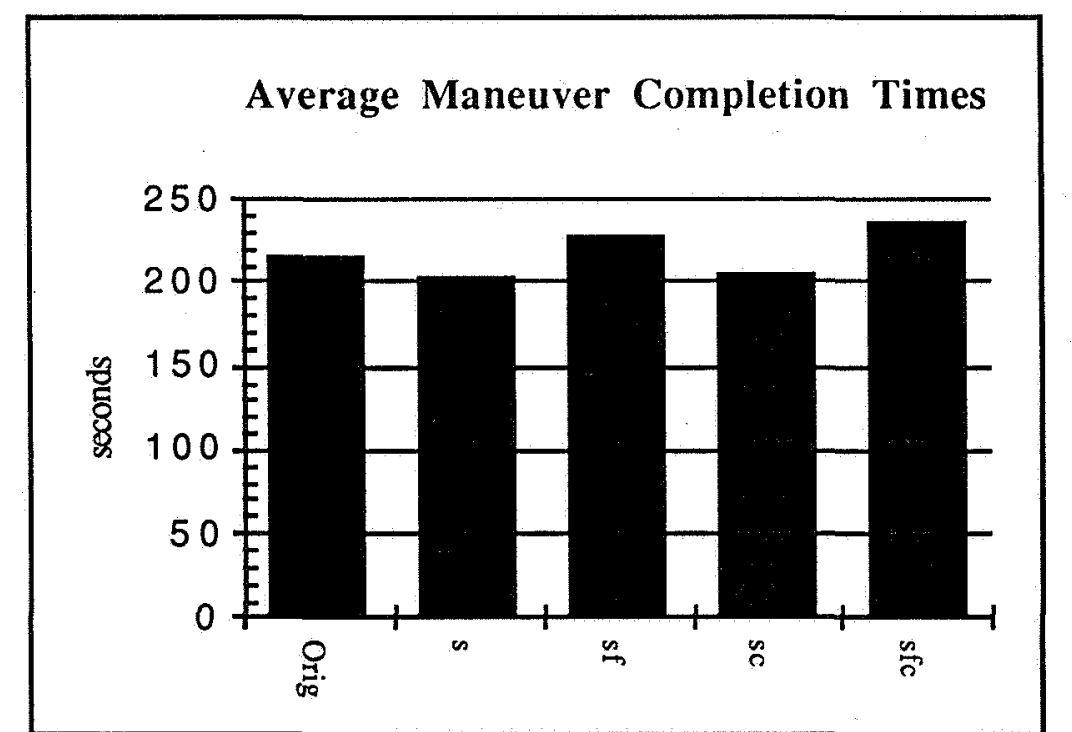

Figure 7.13. Average Completion Time of Maneuvers for Alt Mode

A second difference, which affects the performance of the jet limiting and anti-chatter rule packages, is that the greater thrust of the primary jets leads to far shorter burns and consequently, fewer jet selections per burn. The number of jet switches is a full order of magnitude less than for the vernier jets, Figure 7.12. With fewer jet selections to perform, the fuzzy attitude controller actually performs worse when inhibited by the jet limiting and anti-chatter rules, prevented from picking the best jet selections in terms of acceleration and angle error, especially during attitude hold discussed in the following section. Initial 
impressions may be that the jet limiting rules did save some fuel, but when maneuver completion time is considered, Figure 7.13, it becomes apparent that this gain was made by reducing the total rate change required, rather than finding more efficient jet selections. The anti-chatter package affects the maneuver results very little.
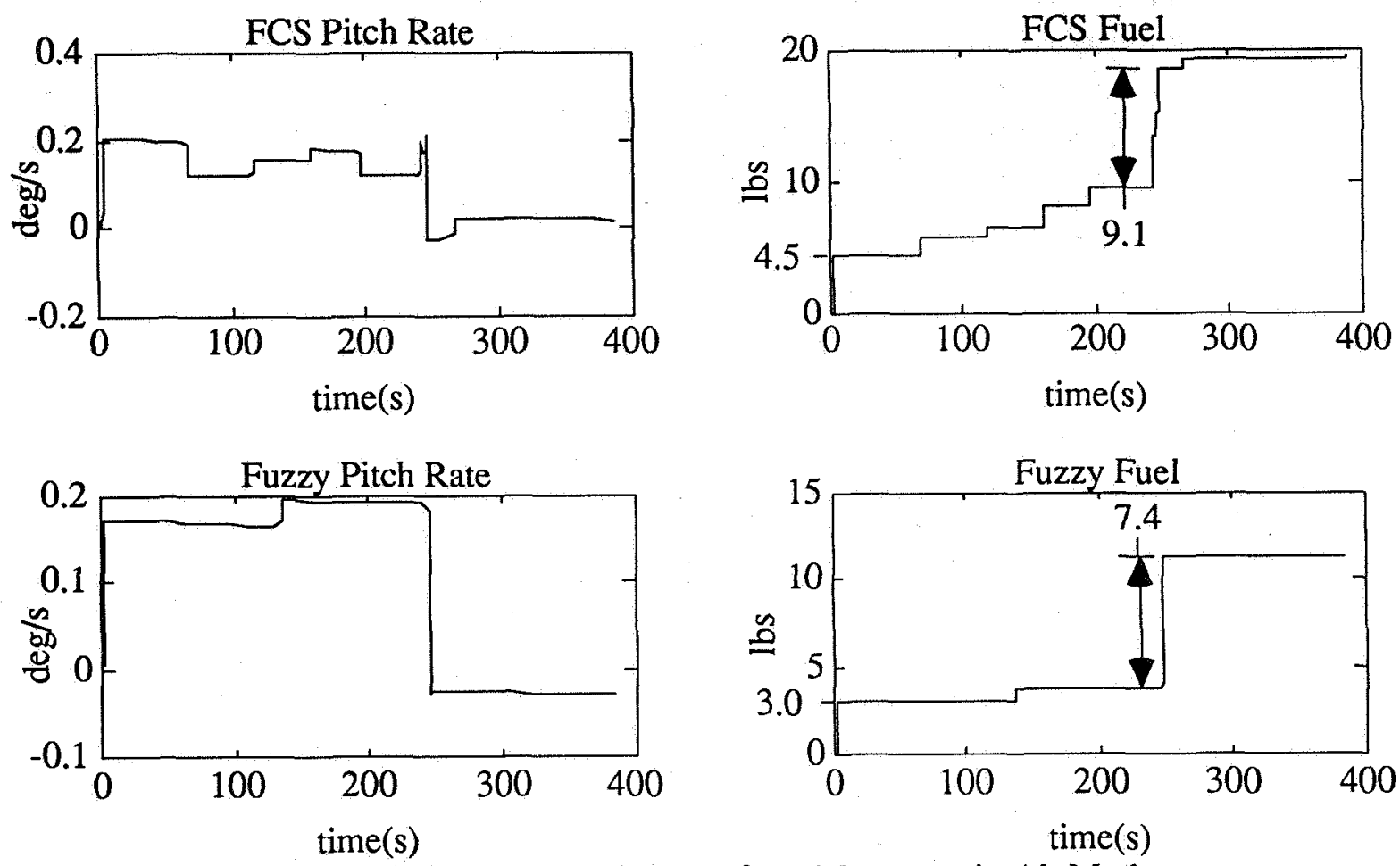

Figure 7.14. Pitch Rate and Fuel Usage for a Maneuver in Alt Mode

For attitude maneuvers, the fuzzy rules do not specify an attitude trajectory along which the attitude must travel as does the FCS (see section 6.5.3). They only require the eigenangle to diminish at a specified rate. This provides greater fuel performance compared to the FCS, which limit cycles about the desired attitude trajectory during a maneuver, Figure 7.14. The fuzzy attitude controller performed only three burns, an initial acceleration burn, one correction burn while coasting through the maneuver, and a deceleration burn. The FCS performed many burns during the maneuver to maintain the required attitude trajectory. For the acceleration and the deceleration burns, the fuzzy attitude controller used significantly less fuel, demonstrating the benefit of the fuzzy jet selection angle error 
minimization and acceleration maximization scheme.

\subsubsection{Inertial Attitude Hold Results}

Criterion similar to those used to evaluate maneuvers are also important when evaluating inertial attitude holds. Rates of fuel usage and jet switches are now the quantities of interest. To ensure comparisons between the FCS and the fuzzy attitude controller are fair, the two should maintain comparable levels of pointing error. Figure 7.17 plots the average eigenangle for the maneuvers. In all cases the fuzzy attitude controller maintained smaller pointing error. This indicates that the lower fuel consumption rates for the fuzzy attitude controller represent true improvements.

Figure 7.17 demonstrates the earlier observations made about the baseline rule package. With no direct means for specifying an attitude hysteresis region, pointing error is quite small. Although this has little affect upon the fuel consumption rate, since the required rate change during attitude hold is dictated only by the disturbance acceleration, it drives the jet switch rates to unacceptably high levels. There is a definite tradeoff between maintaining small pointing error and reducing jet switches.

The addition of the slow disturbance rule package allows the attitude error to excurse a larger region through specification of the pointing error constraint. Limit cycle times, the amount of time between attitude hold firings, Figure 7.17, can be increased dramatically by performing fewer but larger rate changes according to the slow disturbance trajectory command. The decrease in the total number of burns also reduces the jet switch rate. The limit cycle times, all over 300 seconds, are much longer than the required settling time for the transients to die out of the disturbance acceleration estimate. 


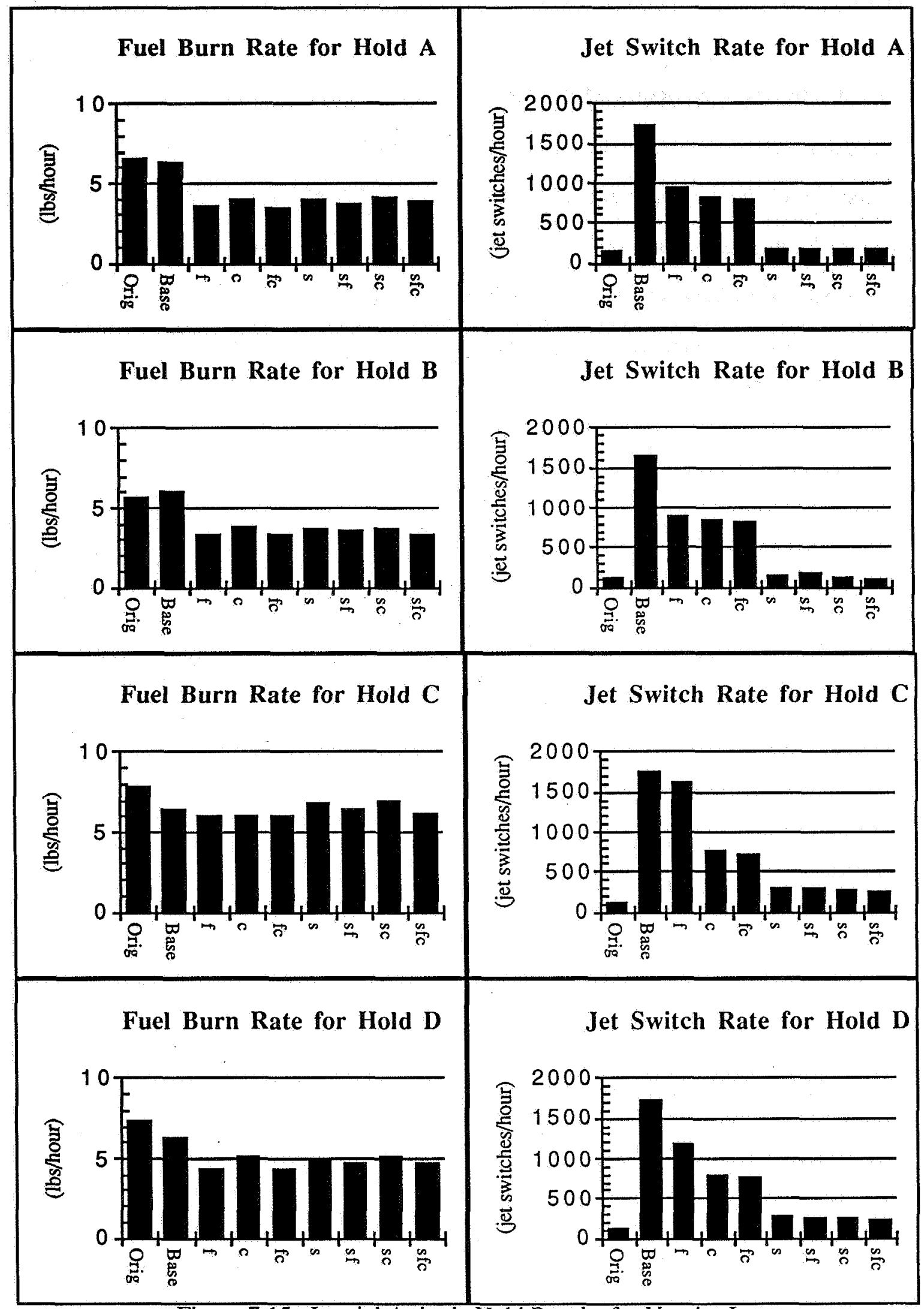

Figure 7.15. Inertial Attitude Hold Results for Vernier Jets 


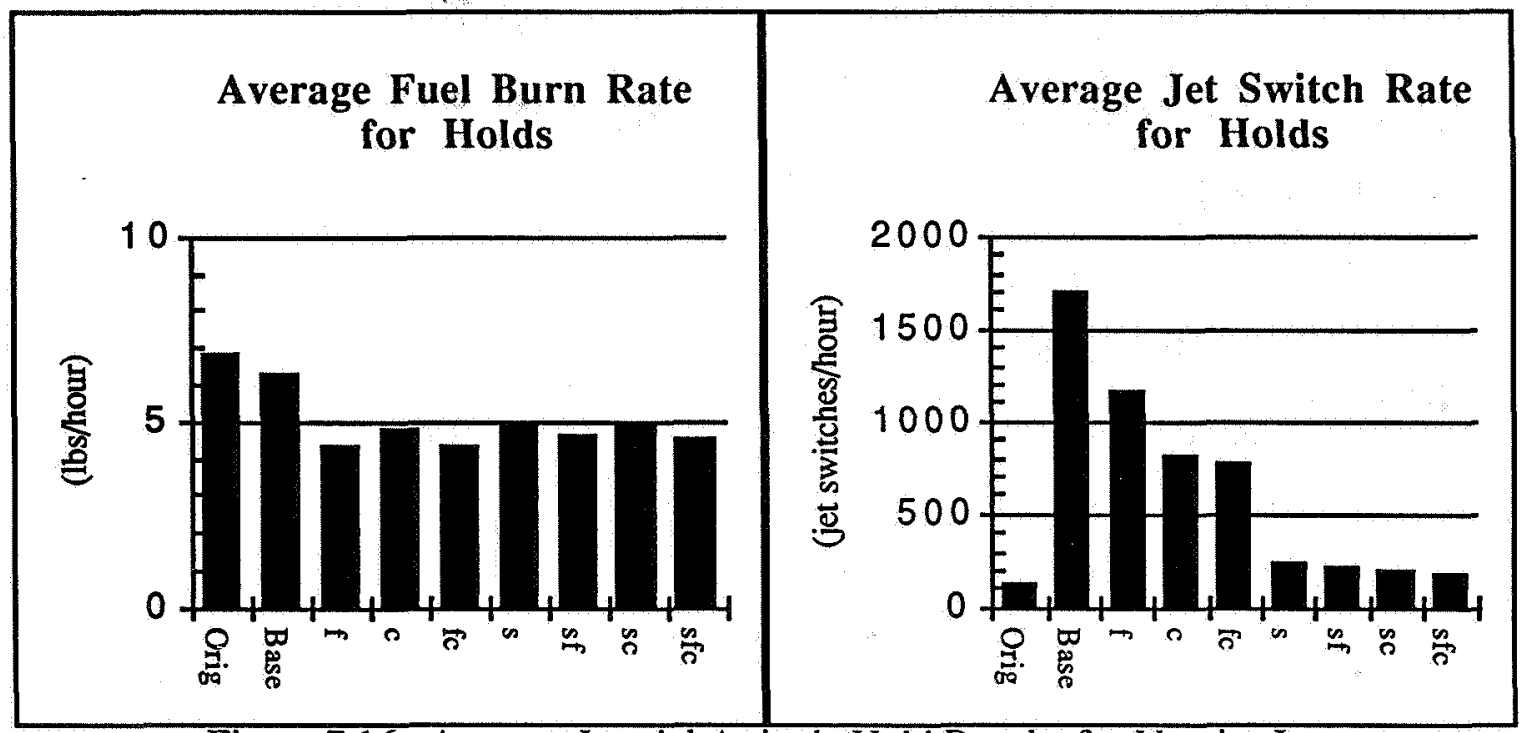

Figure 7.16. Average Inertial Attitude Hold Results for Vernier Jets

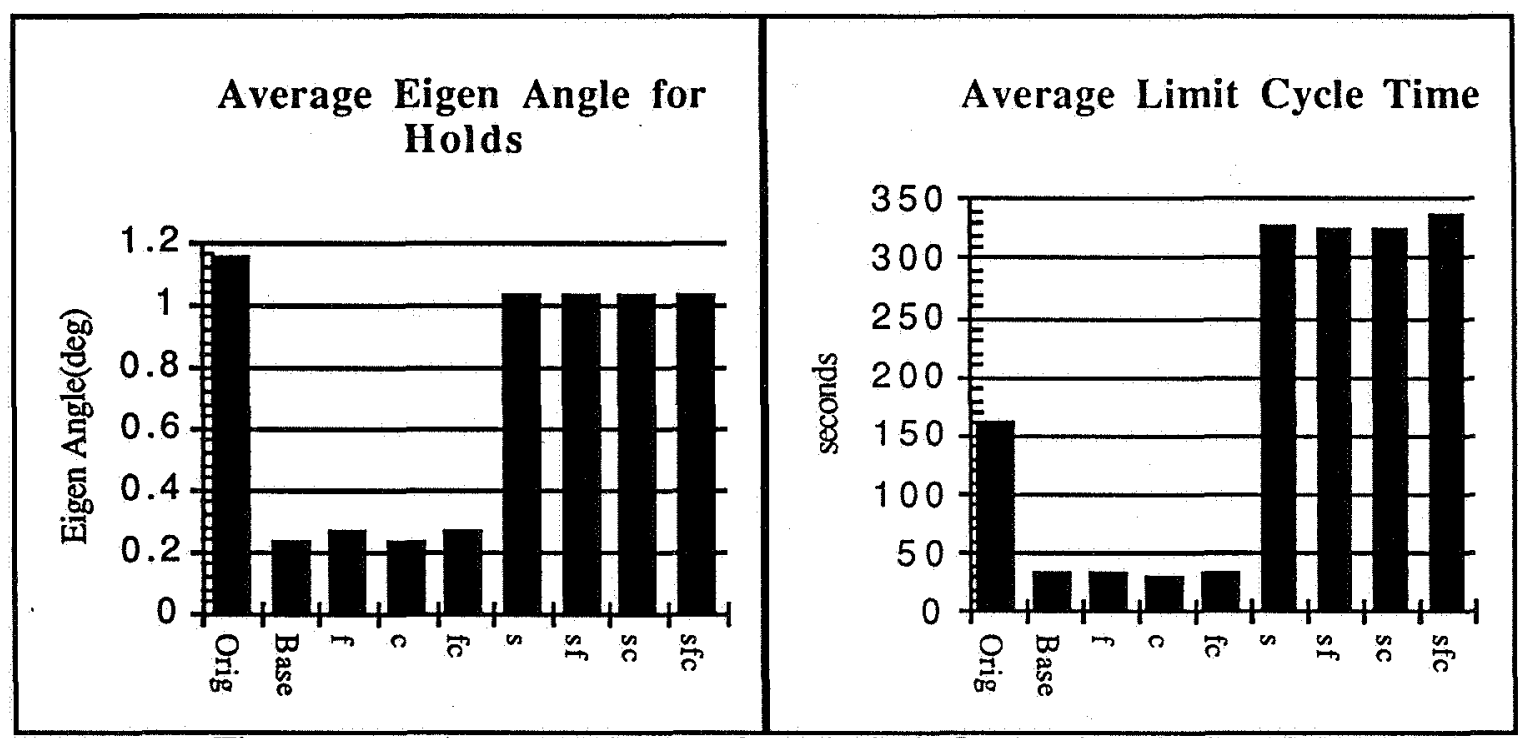

Figure 7.17. Average Eigenangle and Limit Cycle Time for Inertial Attitude Hold using Vernier Jets

Figures 7.18 and 7.19 show body rates for the FCS and fuzzy attitude controller, 'sfc', during hold B. In roll there is a positive disturbance acceleration which switches direction near 3800 seconds. The disturbance acceleration in pitch is negative until about 3600 seconds. In yaw the disturbance is primarily positive until 3600 seconds. The rate changes due to jet firings appear as vertical lines, while the slanted segments result from the disturbance acceleration. 

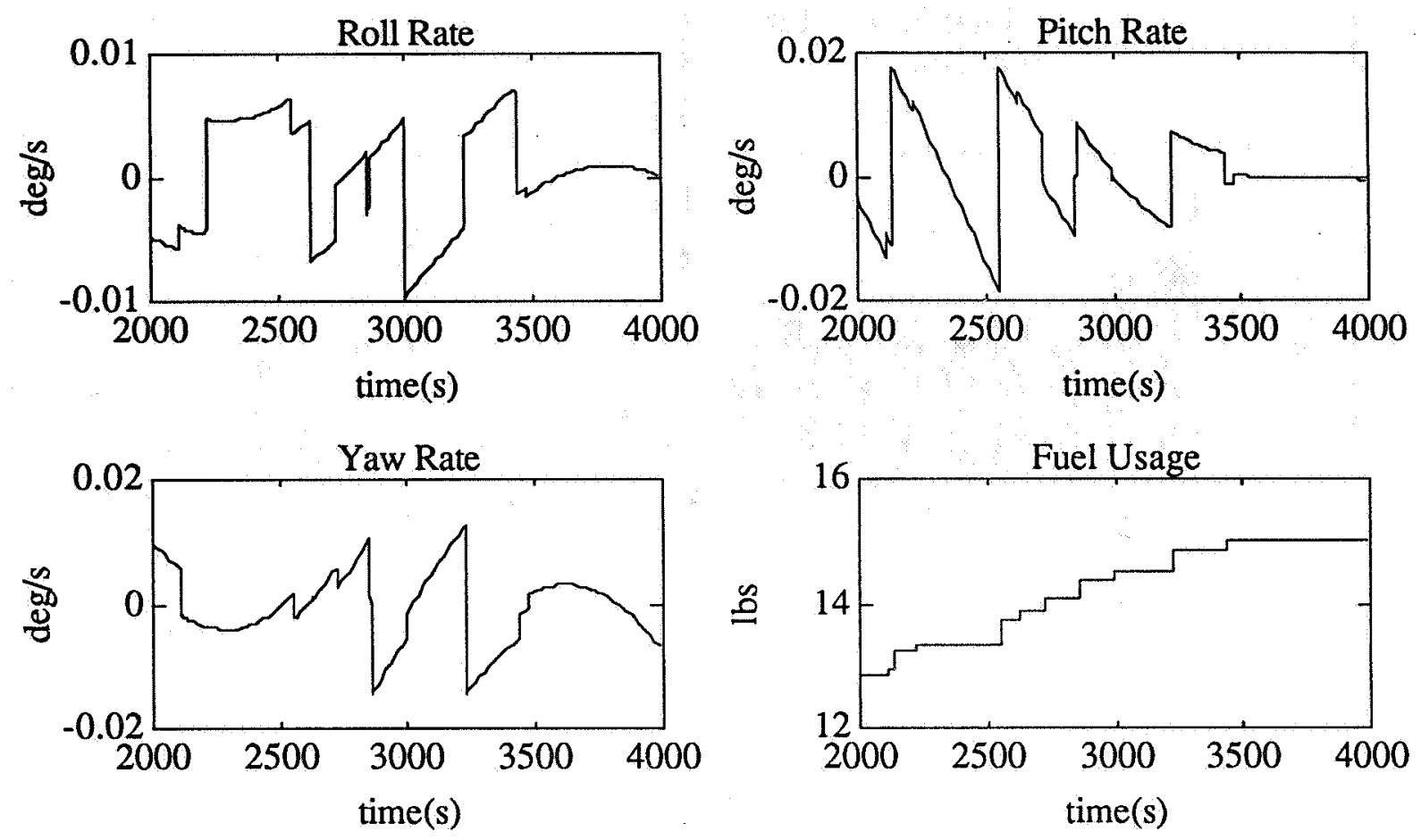

Figure 7.18. FCS Body Rates for a Segment of Hold B using Verniers
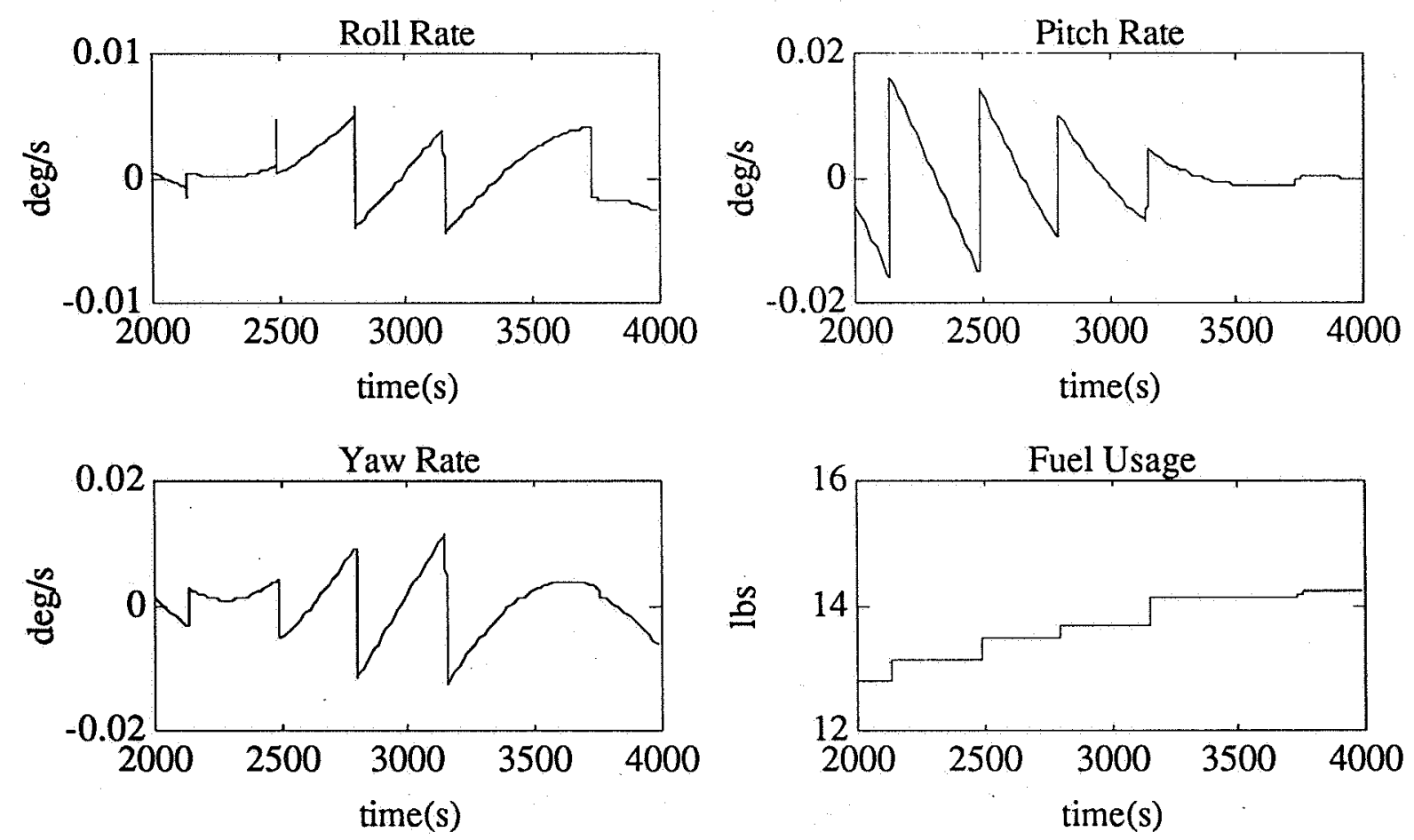

Figure 7.19. Fuzzy Attitude Controller Body Rates for a Segment of Hold B using Verniers 
The benefit of fuzzy's multi-axis approach is apparent when one notes that many of the FCS rate changes do not act to oppose the disturbance acceleration. Rate change in the direction of the disturbance is a waste of fuel. Instead of commanding rate changes on a per-axis basis assuming that a commanded rate change in one axis won't affect another, the fuzzy attitude controller looks at the total error and performs the appropriate rate change in all three axes simultaneously and with greater accuracy due to the jet selection angle error penalty, leading to longer limit cycles and fuel savings since the rate changes consistently oppose the disturbance acceleration.

The following figures present the inertial attitude hold results for Alt Mode. The fuzzy attitude controller results are better than the FCS results in all categories. As for the maneuvers, the jet limiting and anti-chatter rule packages tend to worsen performance. The holds conducted with the anti-chatter rules active demonstrate how inhibiting the controller's ability to change which jets are on and off at the current point in time, can actually lead to a greater number of jet switches later on. Results with these rule packages active are quite inconsistent, Figure 7.20. There does seem to be a symbiotic relationship between the two rule packages. With both activated, Figure 7.21 , performance is better than with either one alone. This is because the jet limiting rules penalize firing multiple jets at once. On average then, with this rule package active, each jet selection will have fewer jets. With fewer jets on, less jet switches are required to turn the jets off, making it easier for the controller to end the burn at the correct time while the anti-chatter rules are active. 


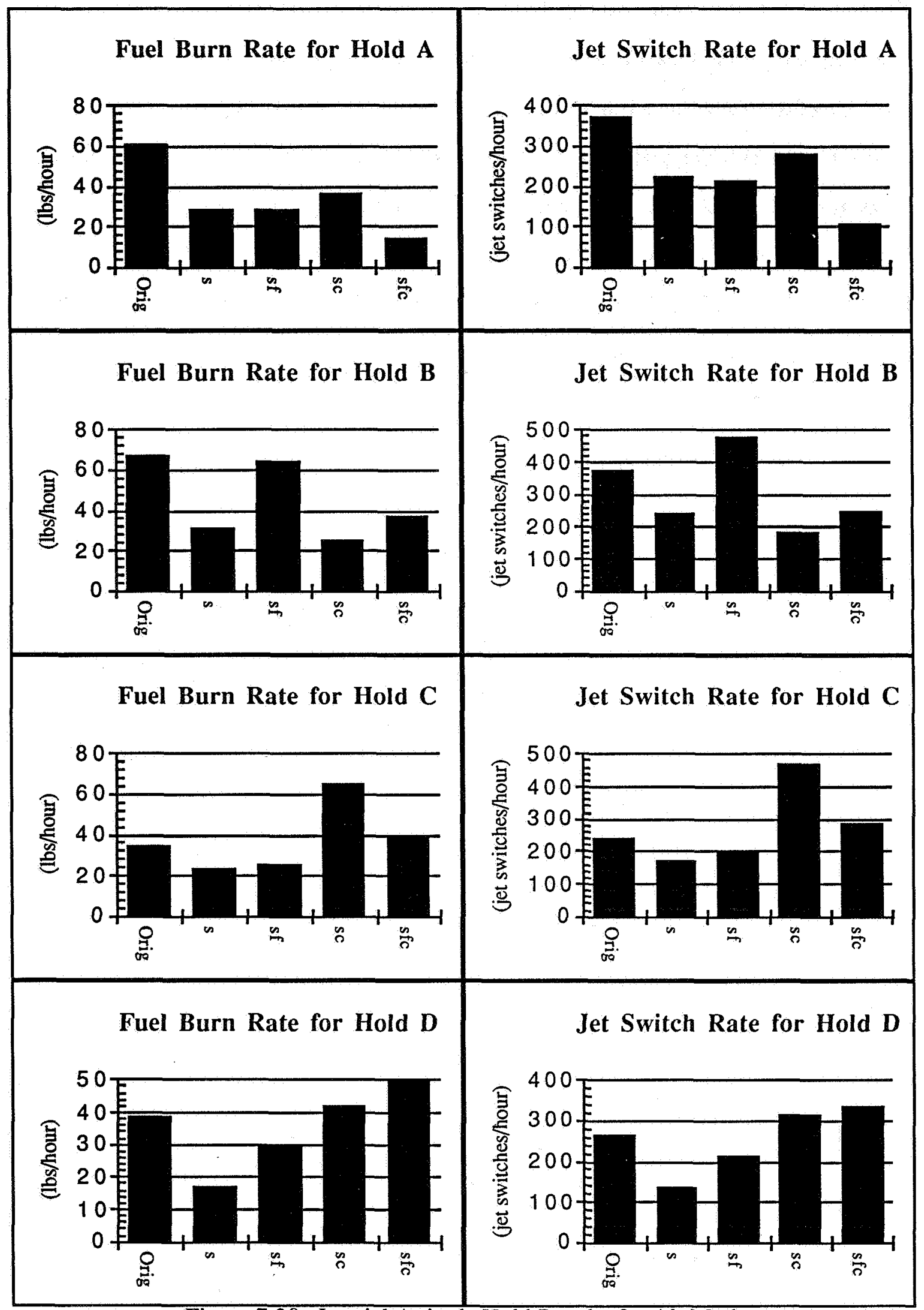

Figure 7.20. Inertial Attitude Hold Results for Alt Mode 


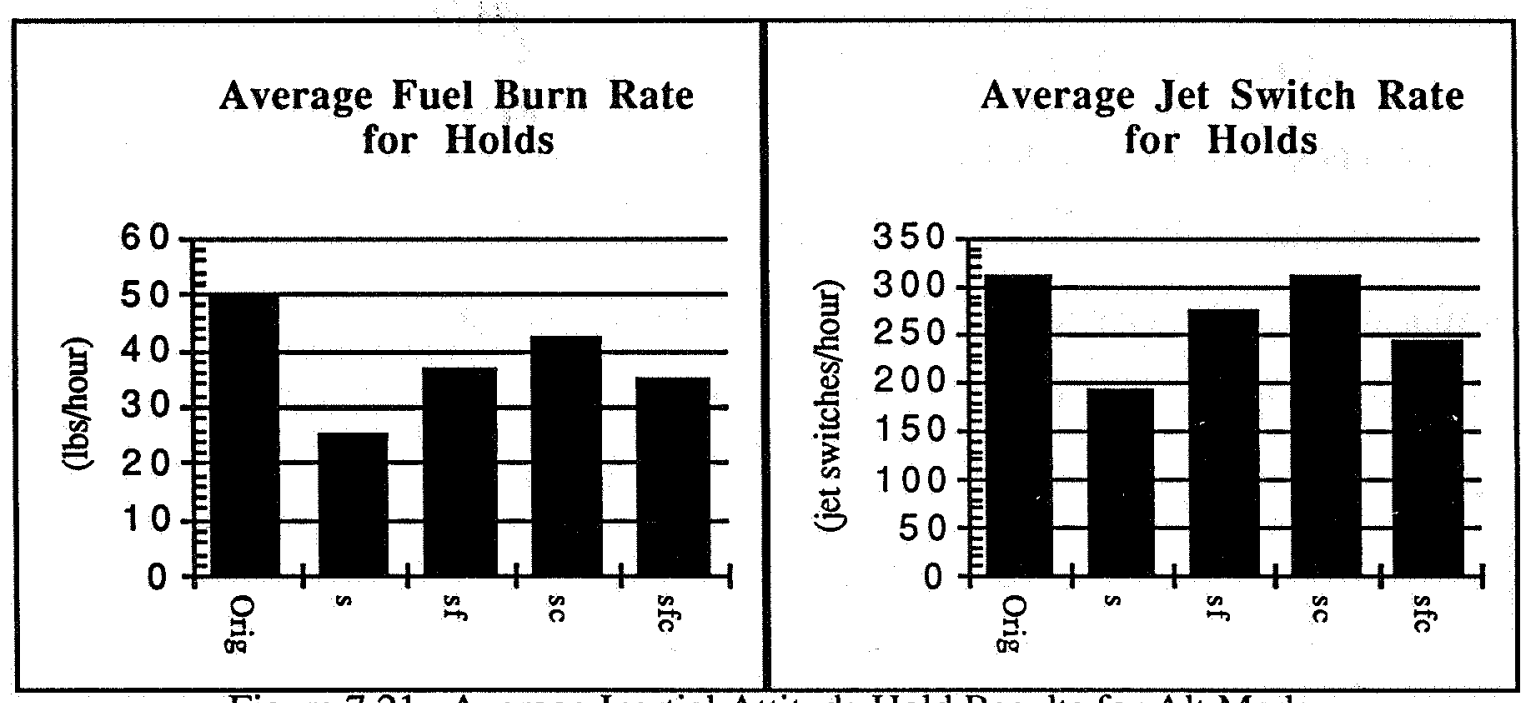

Figure 7.21. Average Inertial Attitude Hold Results for Alt Mode

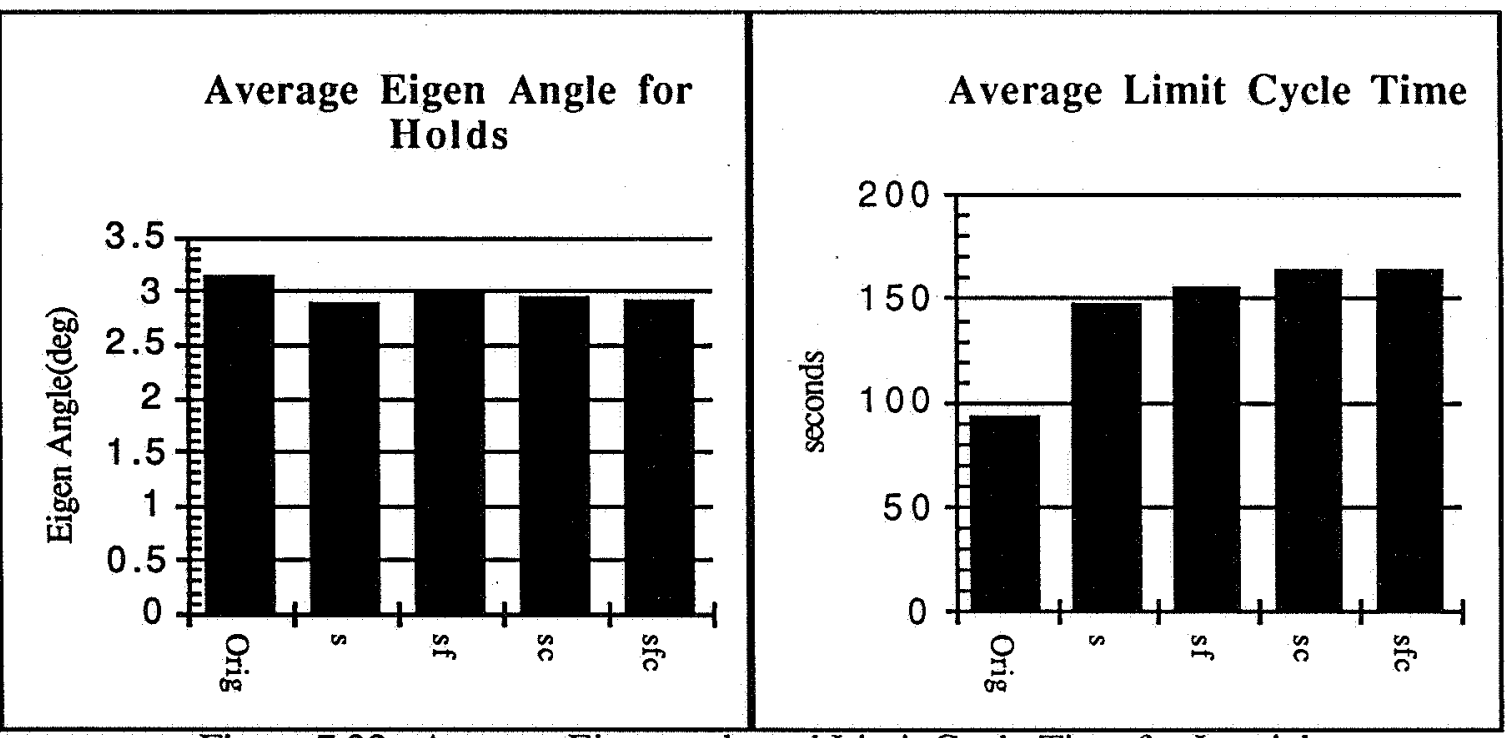

Figure 7.22. Average Eigenangle and Limit Cycle Time for Inertial Attitude Hold using Alt Mode

The limit cycle times for Alt Mode are about half those attained using verniers. Alt Mode's large minimum impulse precludes following the desired slow disturbance trajectory accurately. This, in addition to the small $\beta$ lead to the shorter limit cycles. The limit cycles are still long enough to allow the transients to die out of the disturbance acceleration during the slow trajectory coast. 


\subsubsection{Orbiter with GRO, Modeled as Rigid}

The mass properties of the combined space shuttle and gamma ray observatory present a much more challenging control problem than the space shuttle alone mass properties. The rotational inertia about the roll axis is more than three times as great and the pitch rotational inertia is $30 \%$ greater. To make things worse, the c.g. which normally is maintained above the orbiter centerline, is shifted to starboard nearly a foot, reducing the moment arm available to the jets which provide negative torque about the roll axis, Figure 6.2. These poor mass properties should manifest themselves most in the results for maneuver $D$ which requires a large rotation in roll.

The only parameters modified from the orbiter alone case were the average control acceleration for Vernier jets and the minimum impulses for both verniers and Alt Mode. The Vernier average control acceleration required reduction to accommodate the reduced control authority caused by the combined orbiter-GRO mass properties. The already conservative value for Alt Mode average control acceleration was not reduced. The minimum impulses were reduced with the hope that the slow disturbance trajectories could be acquired with greater accuracy.

Table 7.6. Configurable Parameters for Fuzzy Attitude Controller Orbiter with GRO, Modeled as Rigid, Cases

\begin{tabular}{||c|c|c|}
\hline Parameter & Verniers & Alt. Mode \\
\hline Jet Limiting Package Weight & .2 & .2 \\
\hline $\begin{array}{c}\text { Anti-Chatter Package } \\
\text { Weight }\end{array}$ & .3 & .1 \\
\hline Anti-Flex Package Weight & 0 & 0 \\
\hline $\begin{array}{c}\text { Average Control } \\
\left.\text { Acceleration.(deg/s } \mathrm{s}^{2}\right)\end{array}$ & .003 & .8 \\
\hline Minimum Impulse(deg/s) & .002 & .04 \\
\hline$\beta$ for slow trajectory & .5 & 0 \\
\hline
\end{tabular}




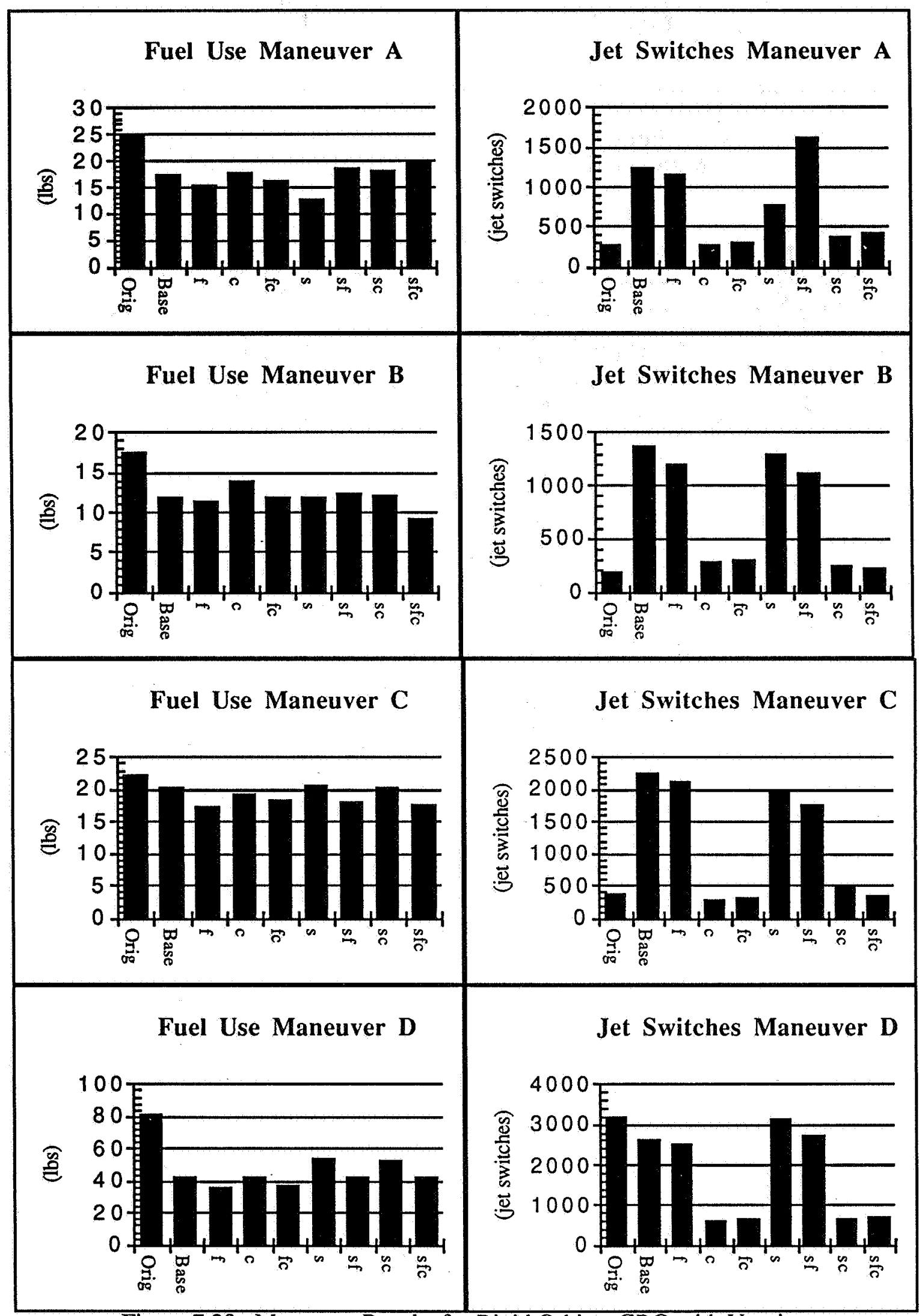

Figure 7.23. Maneuver Results for Rigid Orbiter-GRO with Verniers 


\subsubsection{Maneuver Results}

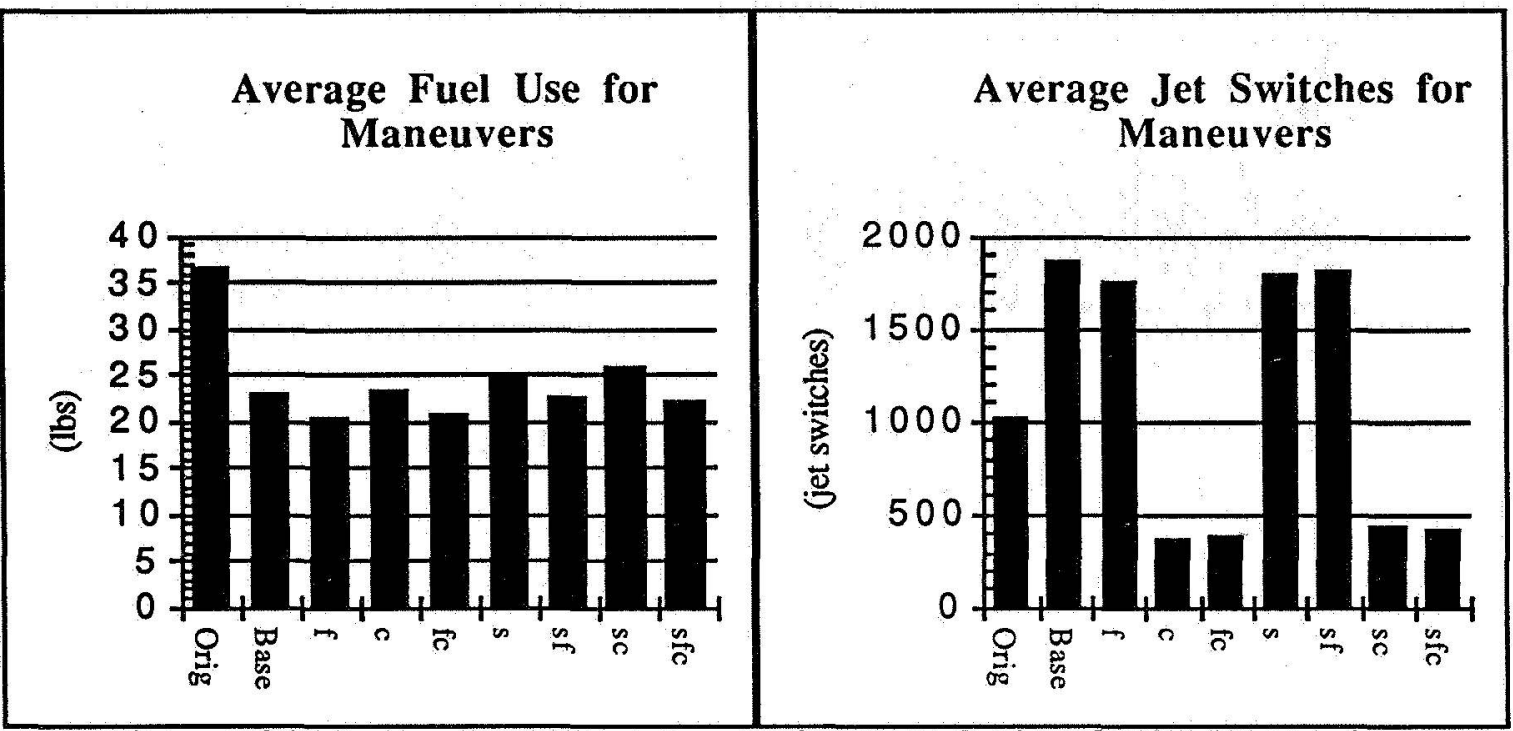

Figure 7.24. Average Maneuver Results for Rigid Orbiter-GRO with Verniers

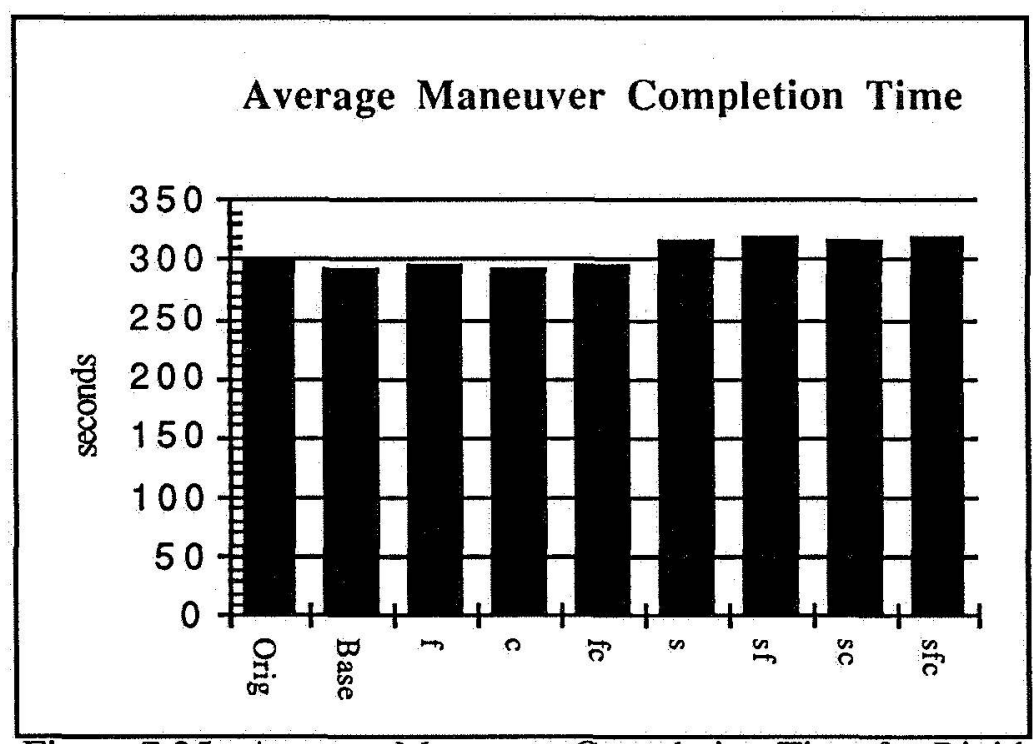

Figure 7.25. Average Maneuver Completion Time for Rigid Orbiter-GRO with Verniers

The maneuver results for vernier jets indicate that having moved away from the nice mass properties of orbiter alone for which the FCS with verniers was designed, FCS performance degrades much more than the fuzzy attitude controller's. For orbiter alone the fuzzy attitude controller had comparable fuel performance with more jet switches, it now averages much less fuel consumption and with the anti-chatter rule package active, fewer jet 
switches than the FCS. As expected, maneuver D, Figure 7.23, was more adversely affected by the change in mass properties than the other maneuvers. Excluding maneuver D, for which the FCS performed quite poorly, the fuzzy attitude controller still improved relative to the FCS from the orbiter alone maneuvers.
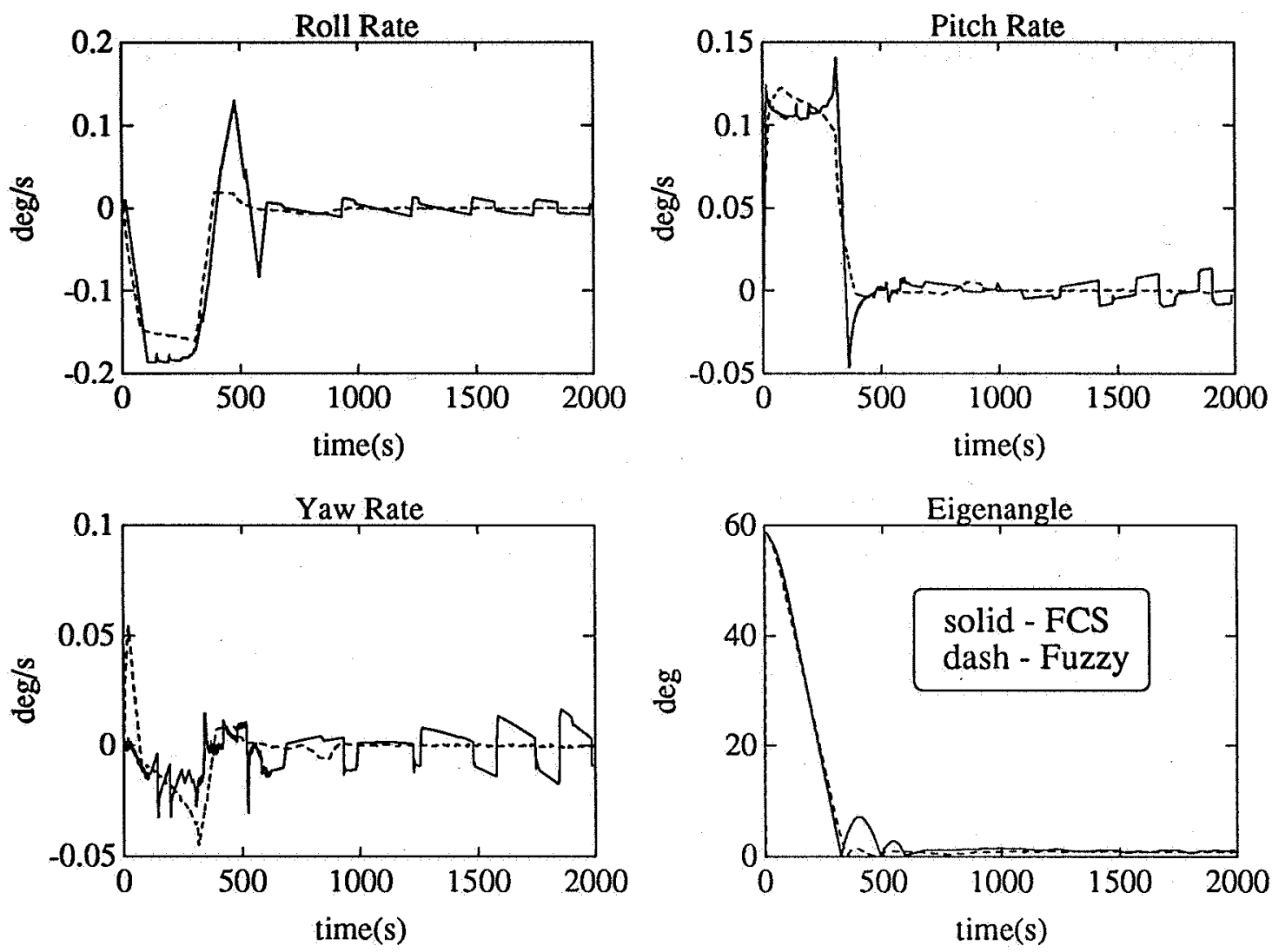

Figure 7.26. Body Rates and Eigenangle for Maneuver D with Verniers for FCS and Fuzzy with Baseline, Jet Limiting, and Anti-Chatter Rules

Time histories from the first 2000 seconds of D are shown in Figure 7.26 and Figure 7.27 for the FCS and the fuzzy attitude controller, 'fc'. From the roll rate and eigenangle plots one can see that the FCS overshoots the desired attitude. Then the FCS maneuver logic, section 6.5.3, initiates another maneuver attempting to build the body rates up to the maneuver rate of $.2 \mathrm{deg} / \mathrm{s}$ in the opposite direction only to overshoot again. This uses up lots of fuel and requires many jet switches. Maneuver cycling such as this highlights one of the weaknesses of the FCS with its crisp switching boundaries. The fuzzy attitude 
controller does not maneuver cycle because its commanded rate is based upon the degree to which the error is small or big. For bigger errors it commands a higher rate, for smaller errors a smaller rate, instead of employing a switch which changes the commanded rate from zero to the maneuver rate when the error crosses a crisp boundary.

These figures also illustrate the fuzzy attitude controller baseline rule package in attitude hold. There is no limit cycling based upon the disturbance acceleration. Attitude error and body rates are kept nearly at zero through frequent jet pulsing. Notice in Figure 7.27, after the attitude hold has begun, the slope of the fuzzy jet switches line is much steeper than that of the FCS due to fuzzy's shorter limit cycles.
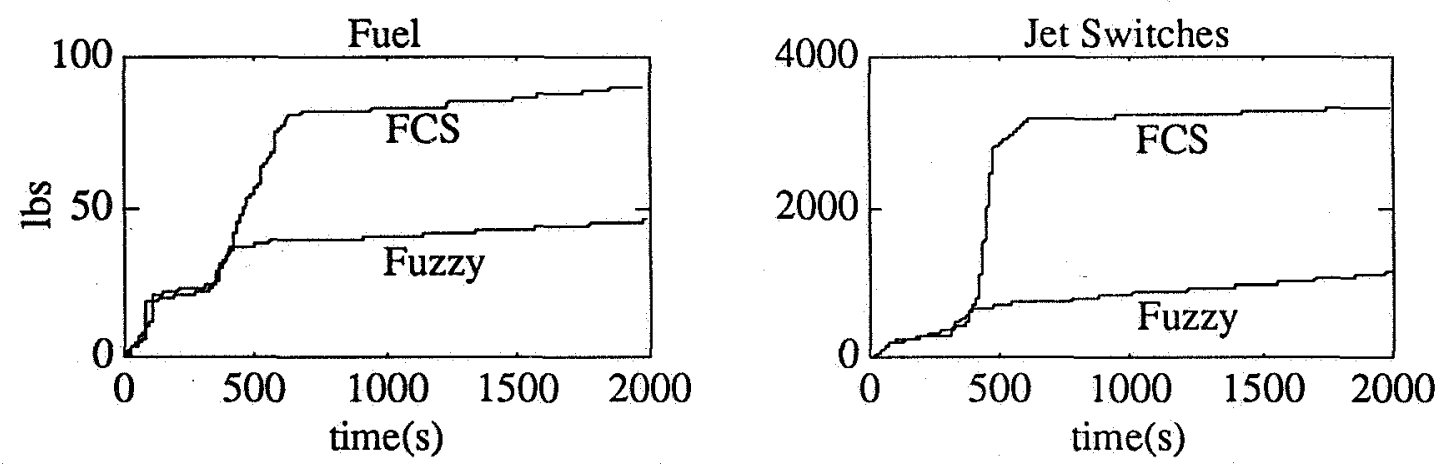

Figure 7.27. Fuel Use and Jet Switches for Maneuver D with Verniers for FCS and Fuzzy with Baseline, Jet Limiting, and Anti-Chatter Rules

The maneuver results for Alt Mode, Figure 7.28, are similar to the orbiter alone case. The fuzzy attitude controller still performs better; however, the margins between the two have reduced. Inexplicably the FCS performed better with these mass properties while the fuzzy controller performed slightly worse. 


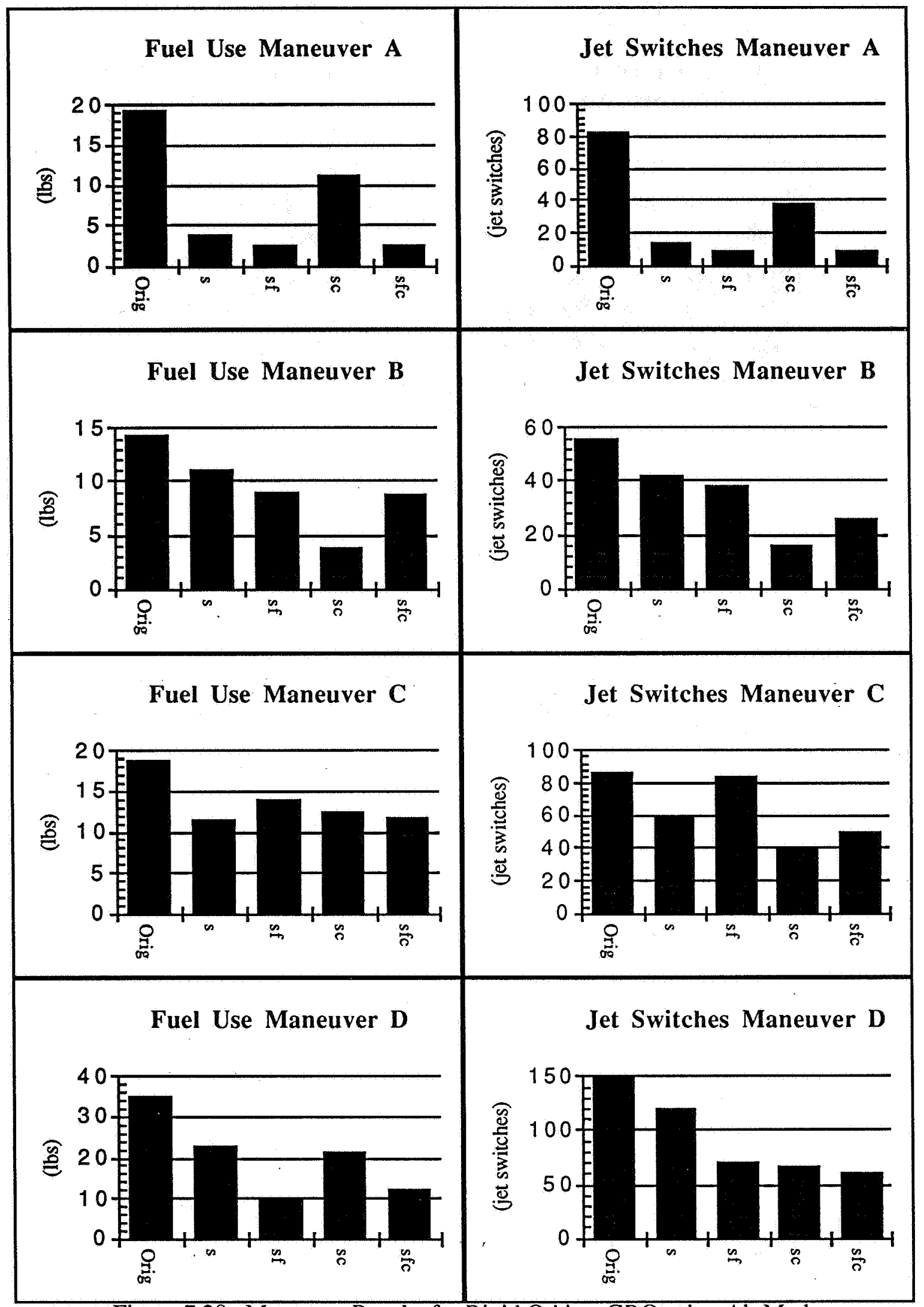

Figure 7.28. Maneuver Results for Rigid Orbiter-GRO using Alt Mode 


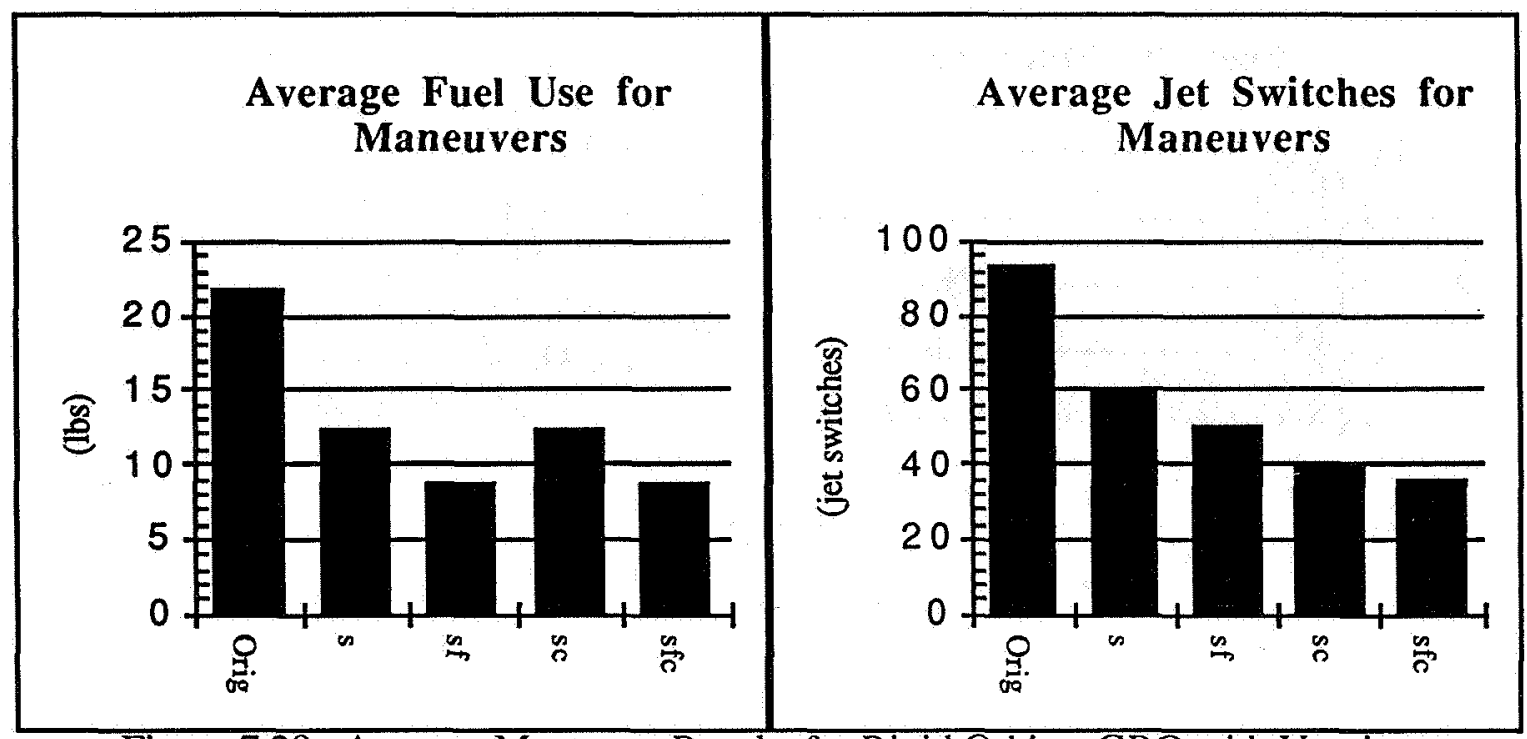

Figure 7.29. Average Maneuver Results for Rigid Orbiter-GRO with Verniers

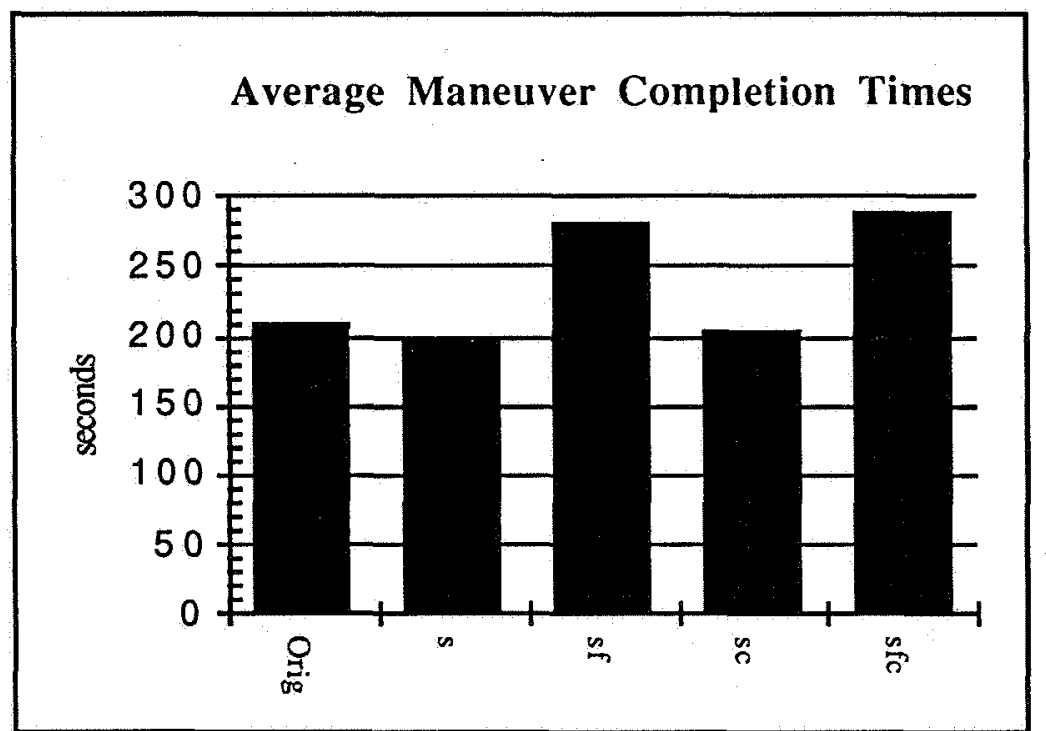

Figure 7.30. Average Maneuver Completion Time for Rigid OrbiterGRO using Alt Mode

The longer burns required by the fuzzy attitude controller to complete these maneuvers (note the increase in average fuel consumption of about $5 \mathrm{lbs}$. between Figure 7.12 and Figure 7.29) point out the kinds of operations for which the jet limiting and anti-chatter rule packages will be beneficial. The longer the burns, the greater the benefit from the additional rule packages due to the greater number of jet selections performed per burn. For maneuver $D$, which required the longest burns, the jet limiting package cut fuel 
consumption in half and the anti-chatter rules lowered the number of jet switches slightly, where in the orbiter alone case they had actually increased the quantity of jet switches.

\subsubsection{Inertial Attitude Hold Results}

There was not as great a differentiation in relative performance between the FCS and fuzzy attitude controllers in switching mass properties for the attitude holds as there was for the maneuvers. The combined mass properties present their greatest challenge when large rate changes must be performed since there is little control authority in some axes.

The fuel consumption rate for the Vernier jets more than doubled on average compared to the orbiter alone case due to the larger rotational inertia. The fuzzy attitude controller has a lower fuel burn rate while maintaining less pointing error than the FCS. The FCS still performs slightly better in terms of jet switches. In the slow trajectory with anti-chatter rule package runs, there is a significant fuel penalty to achieve the fewer jet switches, Figure 7.32; however, with the addition of the jet limiting package this penalty is removed without affecting the jet switch rate.

The goal of more accurately attaining the slow disturbance acceleration trajectory by lowering the minimum impulse was not realized. The average limit cycle times for the runs with the slow disturbance rules active are essentially unchanged from the previous case where a minimum impulse $150 \%$ larger was used. This indicates that the degree of accuracy with which the slow trajectory was attained was already sufficiently high that there was little or no improvement to be made. 


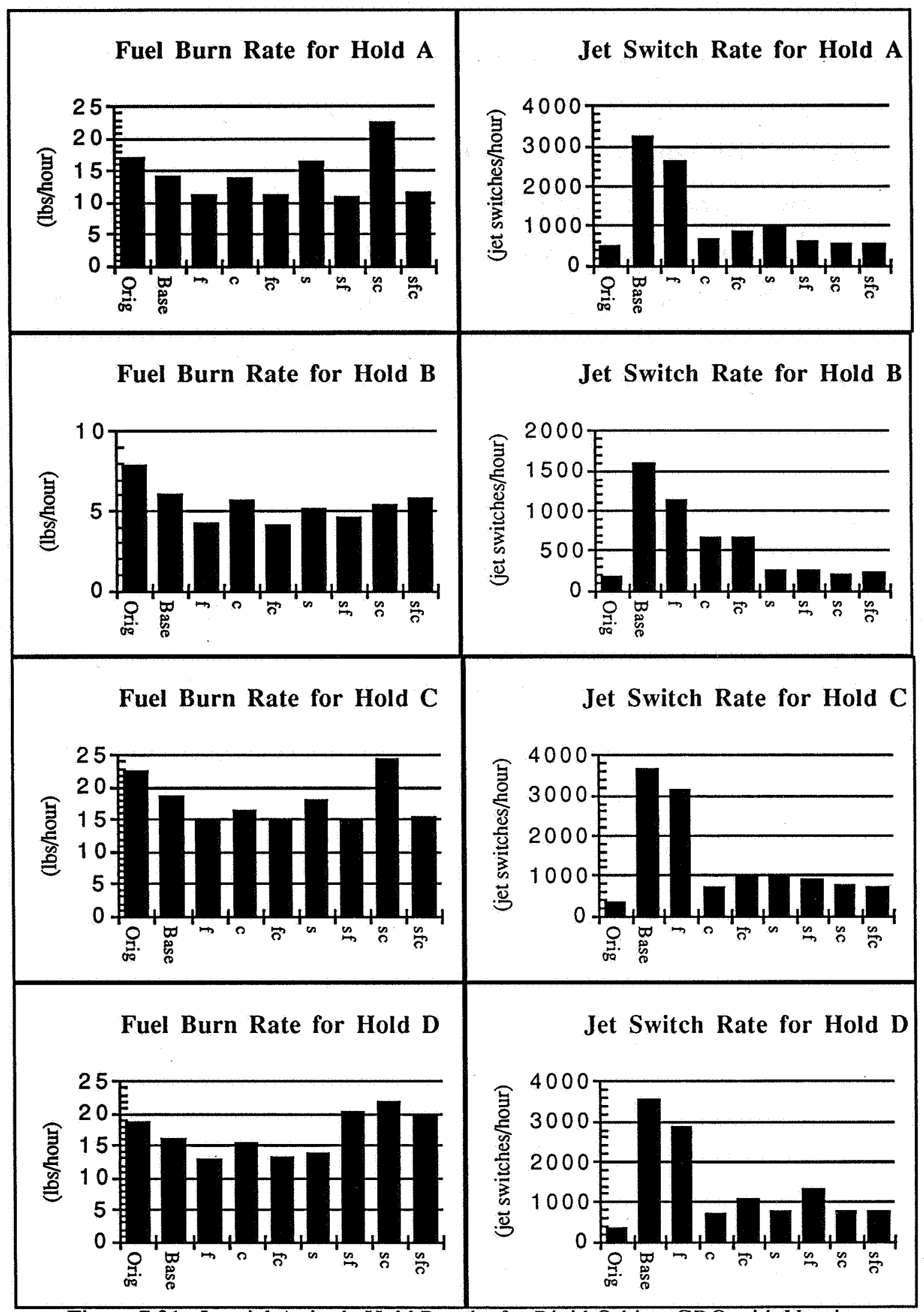

Figure 7.31. Inertial Attitude Hold Results for Rigid Orbiter-GRO with Verniers 


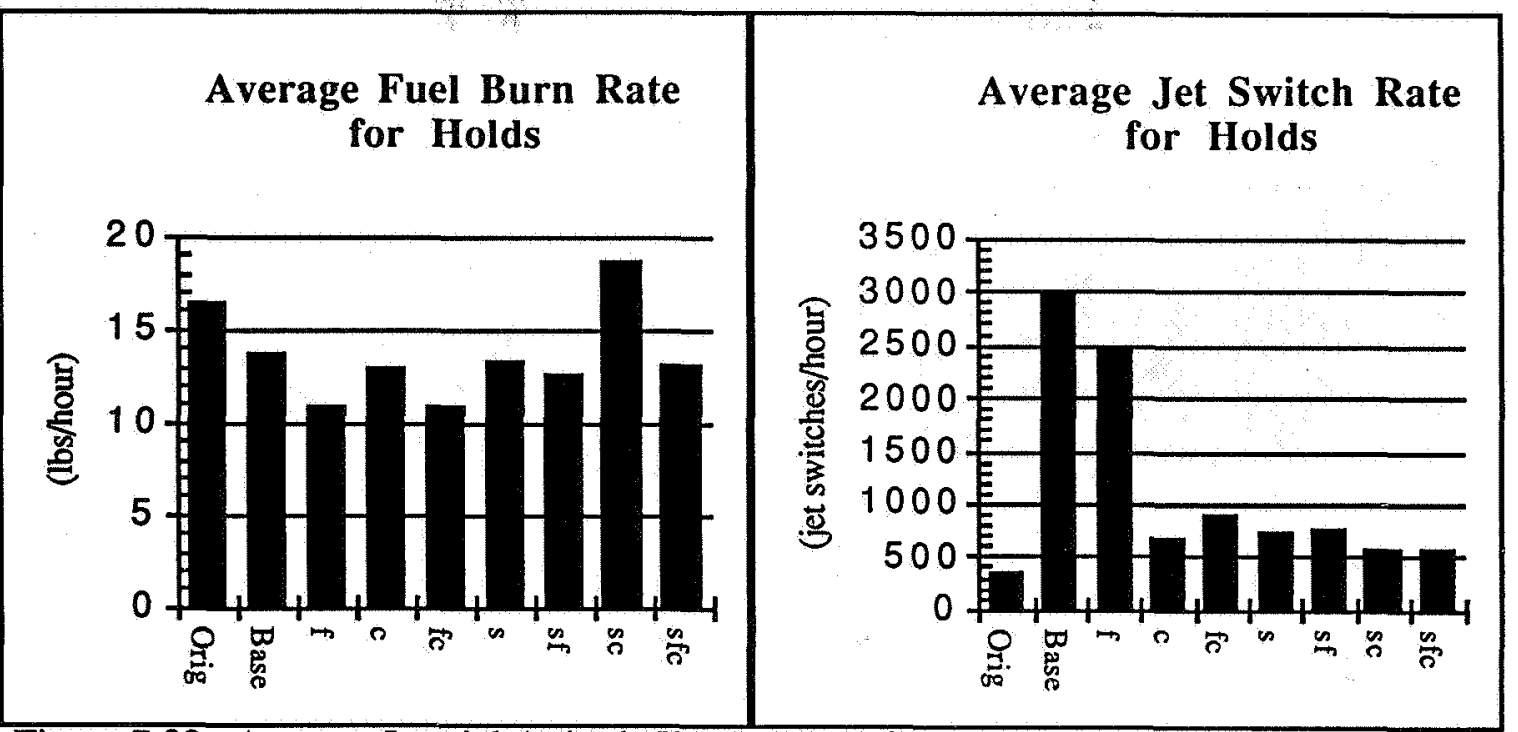

Figure 7.32. Average Inertial Attitude Hold Results for Rigid Orbiter-GRO with Verniers

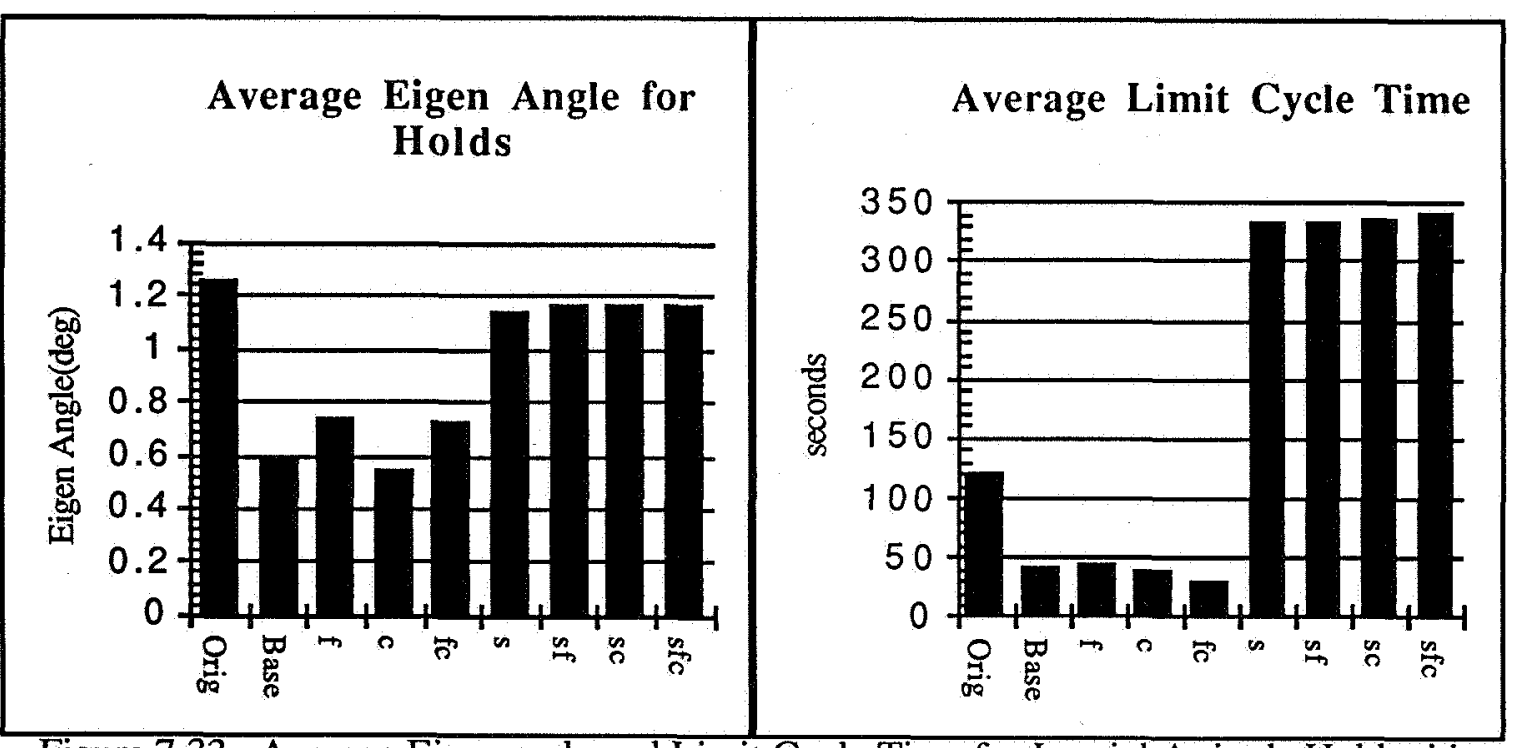

Figure 7.33. Average Eigenangle and Limit Cycle Time for Inertial Attitude Hold with Rigid Orbiter-GRO and Verniers

As was the case for the maneuvers, performance for the FCS using Alt Mode improved for the orbiter-GRO mass. Although the fuzzy attitude controller performance was quite similar for the orbiter-GRO mass as for the orbiter alone case, it still burned fuel at about $50 \%$ the rate of the FCS using fewer jet switches and maintaining nearly identical levels of pointing error. 


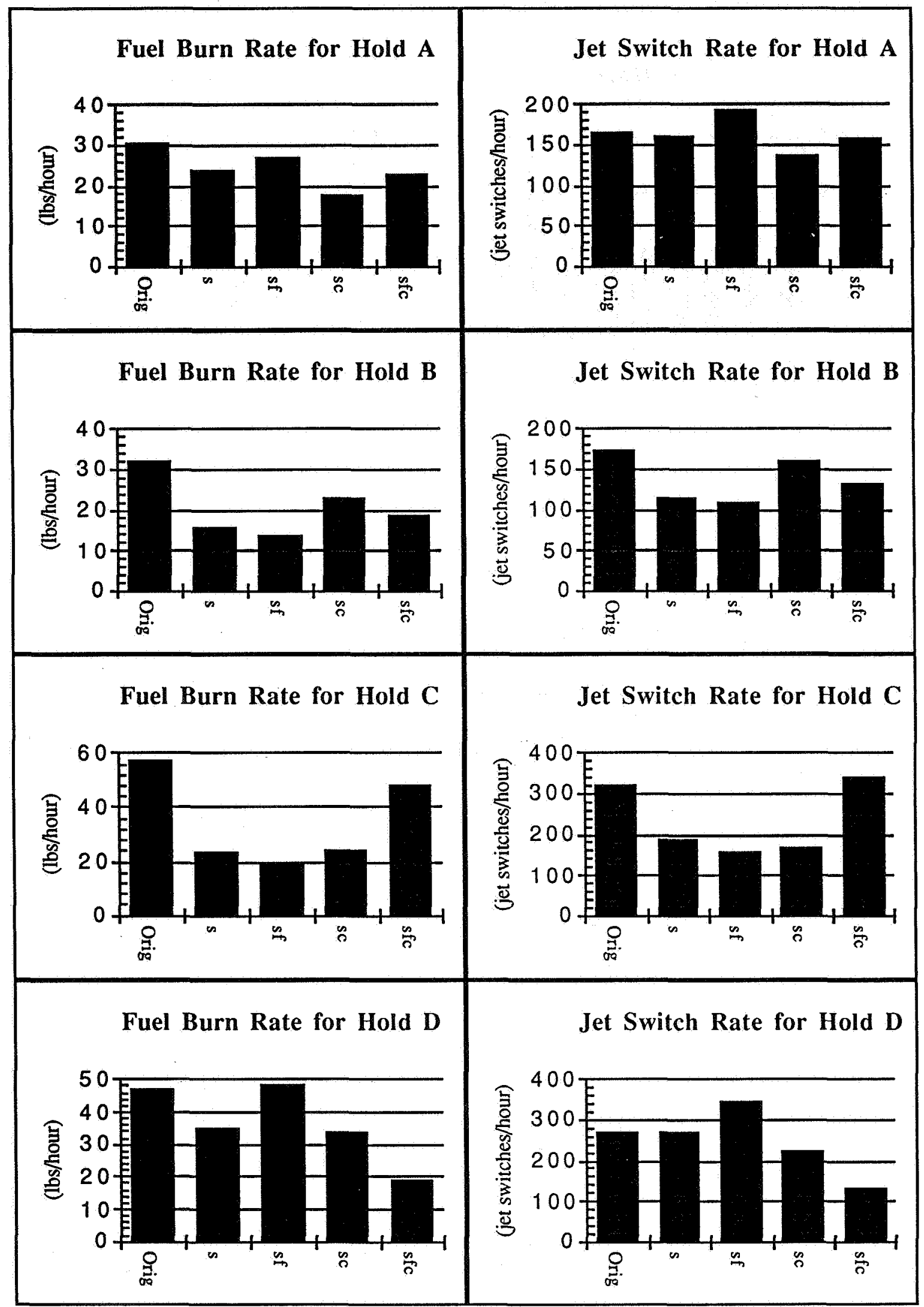

Figure 7.34. Inertial Attitude Hold Results for Rigid Orbiter-GRO with Alt Mode 


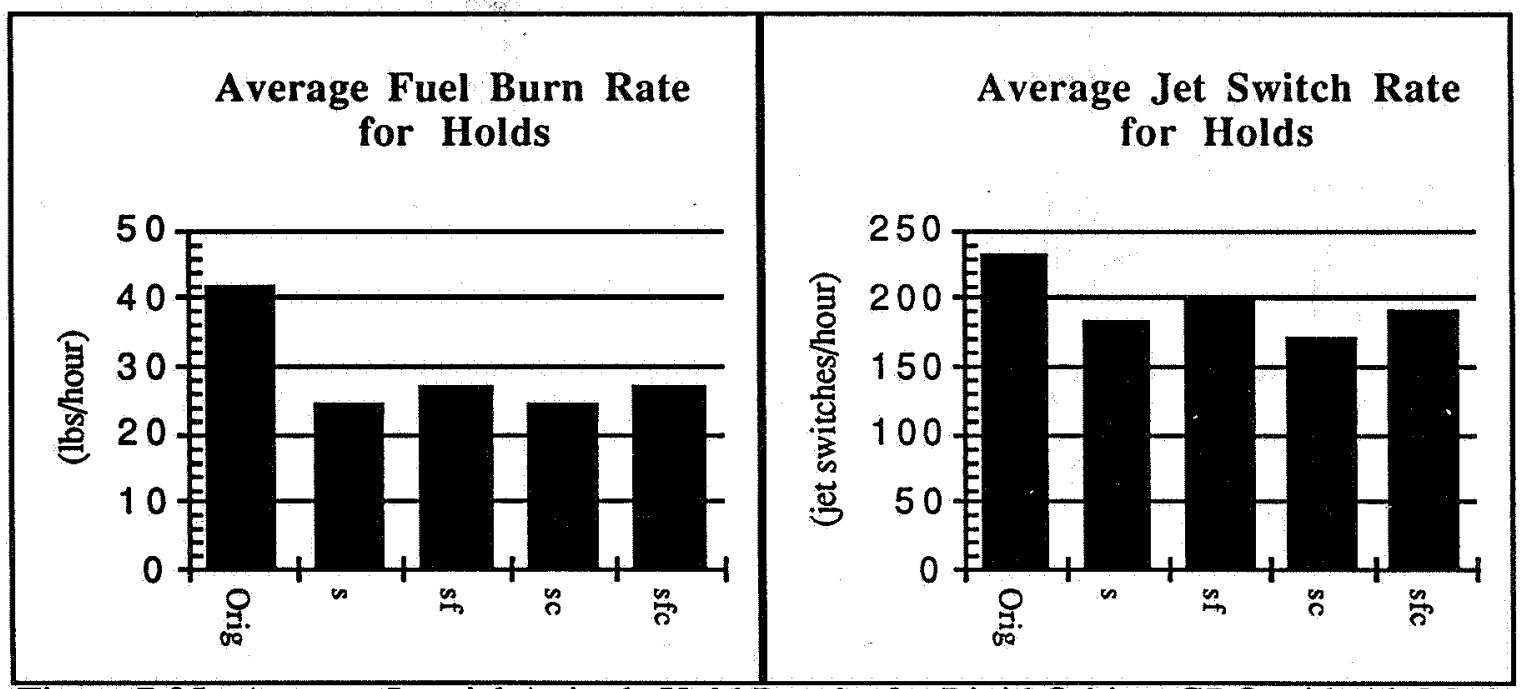

Figure 7.35. Average Inertial Attitude Hold Results for Rigid Orbiter-GRO with Alt Mode

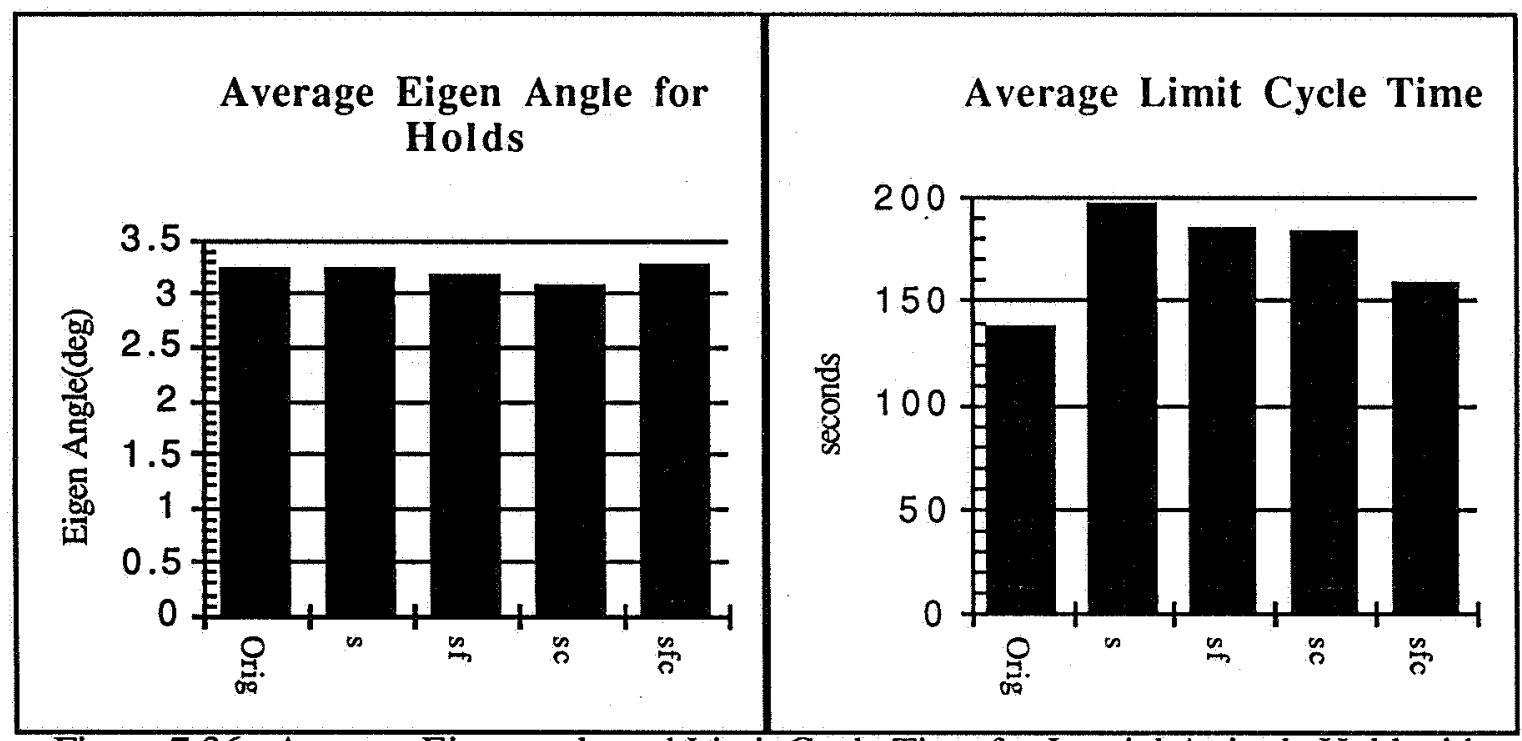

Figure 7.36. Average Eigenangle and Limit Cycle Time for Inertial Attitude Hold with Rigid Orbiter-GRO and Alt Mode

As indicated by the increase in average limit cycle time, Figure 7.36 , the effort to more accurately acquire the slow disturbance acceleration trajectory by decreasing the minimum impulse was successful. The level of accuracy, however, is still quite poor. The rate change from one $80 \mathrm{msec}$ firing in Alt Mode is often so large that the entire error envelope is excursed even if the disturbance acceleration is acting in the opposite direction. 

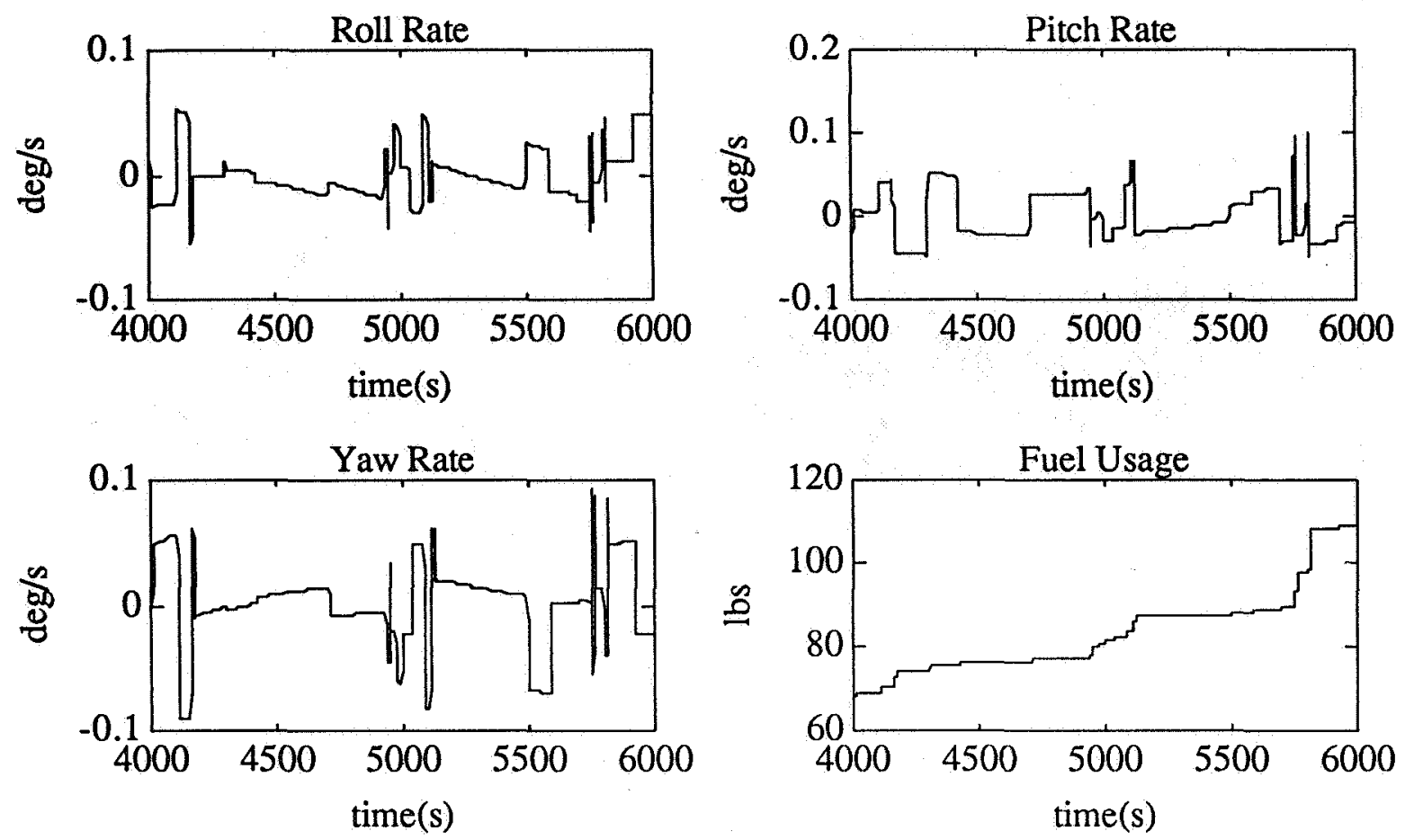

Figure 7.37. FCS Body Rates for a Segment of Hold C, Alt Mode
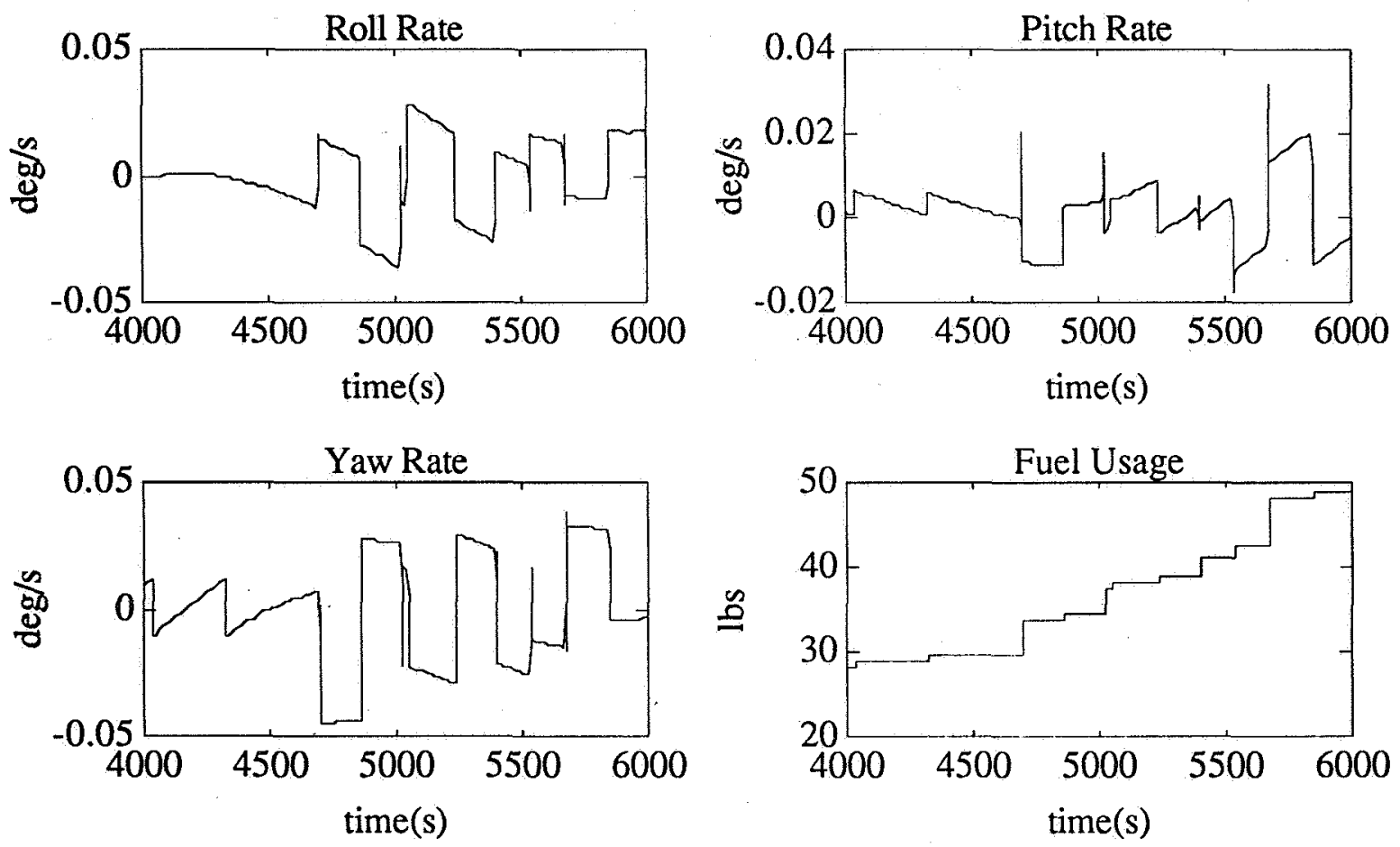

Figure 7.38. Fuzzy Attitude Controller Body Rates for a Segment of Hold C, Alt Mode 
Figure 7.37 and Figure 7.38 show body rates during an inertial attitude hold for the FCS and the fuzzy attitude controller using only the slow disturbance rule package. Neither controller does a good job of achieving a one sided limit cycle as indicated by the many rate changes which occur in the same direction as the disturbance. The fuzzy controller does manage to do a fairly good job for the first 700 seconds shown, then as the disturbance acceleration starts to change directions in yaw, firings begin to occur in both directions indicating that the entire error envelope is being excursed before the rate can be reversed.

The FCS, Figure 7.37, has much greater difficulty achieving an efficient limit cycle. The inter-axis coupling is far too great for the single axis approach. When a firing is commanded in one axis, this pushes another axis into the firing region of its phase plane causing another firing, and so forth. This is worsened by the lack of an attempt to reduce angle error in the jet selection in favor of maximizing acceleration. The result is more firings and greater fuel consumption.

\subsubsection{Orbiter with GRO, Modeled as Flexible}

This section presents results from 2000 second simulations which modeled the orbiterRMS-GRO system as flexible (flexible RMS). Full 6000 second runs were not possible. In order to capture the flexible dynamics, the sample rate for the output data had to be much higher than for the rigid cases.

The purpose of this section is to demonstrate that by adding additional rules to the jet selection process, specifically the anti-flex rule package, that the excitation of flexible modes can be reduced. Flex rate output 2-norms were calculated for each axis during maneuvers and attitude holds. The 2-norm from the axis with the greatest 2-norm was used as a measure of the anti-flex performance. Lower values of the 2-norm indicate less excitation of the flex modes. 
Two different shuttle FCS configurations were used for the simulations not employing the fuzzy controller, both of which exercise passive means of dealing with flexibility. The first configuration reflects the approach currently used during space shuttle operations with flexible structures. For these runs only attitude measurements were used. The attitude measurements were passed through notch filters, as described in section 6.5 .2 , on the first two flex modes before being passed to the rate estimators. This approach is designed to maintain stability by preventing rate information at the frequencies of the primary flexible modes from entering the rate estimator which has large phase lag at these frequencies. The second configuration used a rate measurement which in turn was used to estimate rigid rate, as described in section 7.1.2. Both configurations attempt to make the plant appear rigid to the controller to prevent the controller from responding to the flexible dynamics.

Essentially the controller will think it is controlling the rigid orbiter-GRO system to the limit of the filter performance.

All fuzzy attitude controller simulations used the rigid rate estimate. Runs were conducted with either the baseline and or the slow disturbance rules active and then with the anti-flex rules in different combinations with the jet limiting and anti-chatter rules, allowing the decrease in the 2-norm of the flex rates due to the anti-flex rule package to be measured.

The values for the fuzzy configurable parameters are listed in table 7.7. Good values for the weight on the output of the anti-flex rule package were found by conducting a few simulations with different weights and choosing the best one. If the weight is chosen too large performance will worsen because the controller begins to pick jets based solely upon how they will affect the flexible modes. This increases the number of firings in the long run to achieve a commanded rate change, ultimately leading to greater excitation of the flexible modes. Furthermore, the average control accelerations for verniers and Alt Mode were decreased to reflect the diminished control authority available due to the action of the anti-flex rules. For Alt Mode the average control acceleration was reduced much more than 
required to measure how performance is affected by setting this value conservatively. For verniers the other rule package weights were cut in half to provide the controller with greater flexibility to achieve its anti-flex goals. It is important to remember that there is no golden rule for picking these weights. Each supplemental rule package inhibits the controller in some manner. Reducing the weights gives the controller greater freedom to meet other objectives.

Table 7.7. Configurable Parameters for Fuzzy Attitude Controller Orbiter with GRO, Modeled as Flexible, Cases

\begin{tabular}{||c|c|c|}
\hline Parameter & Verniers & Alt. Mode \\
\hline Jet Limiting Package Weight & .1 & .2 \\
\hline $\begin{array}{c}\text { Anti-Chatter Package } \\
\text { Weight }\end{array}$ & .15 & .1 \\
\hline Anti-Flex Package Weight & .5 & .2 \\
\hline $\begin{array}{c}\text { Average Control } \\
\left.\text { Acceleration.(deg } / \mathrm{s}^{2}\right)\end{array}$ & .002 & .4 \\
\hline Minimum Impulse(deg/s) & .002 & .045 \\
\hline$\beta$ for slow trajectory & .5 & 0 \\
\hline
\end{tabular}

Results are first given for verniers and then for Alt Mode from the 2000 second simulations initiated with the four maneuvers listed in table 7.1. Figure 7.39 shows the 2-norm of the flex rates for each maneuver and subsequent hold using verniers. The runs with the antiflex rule package active, denoted by ' $m$ ', all achieved significant reductions. The average of the 2-norms over all maneuvers, Figure 7.40, shows a reduction of four times over the current shuttle FCS, described as configuration one earlier and denoted by 'Orig1'. Simply supplying the FCS with a rigid rate estimate, 'Orig2' shows some improvement over the current shuttle FCS, but not nearly as much as when jet selections are actively pursued which will avoid exciting the flexible modes or damp modes already excited. 


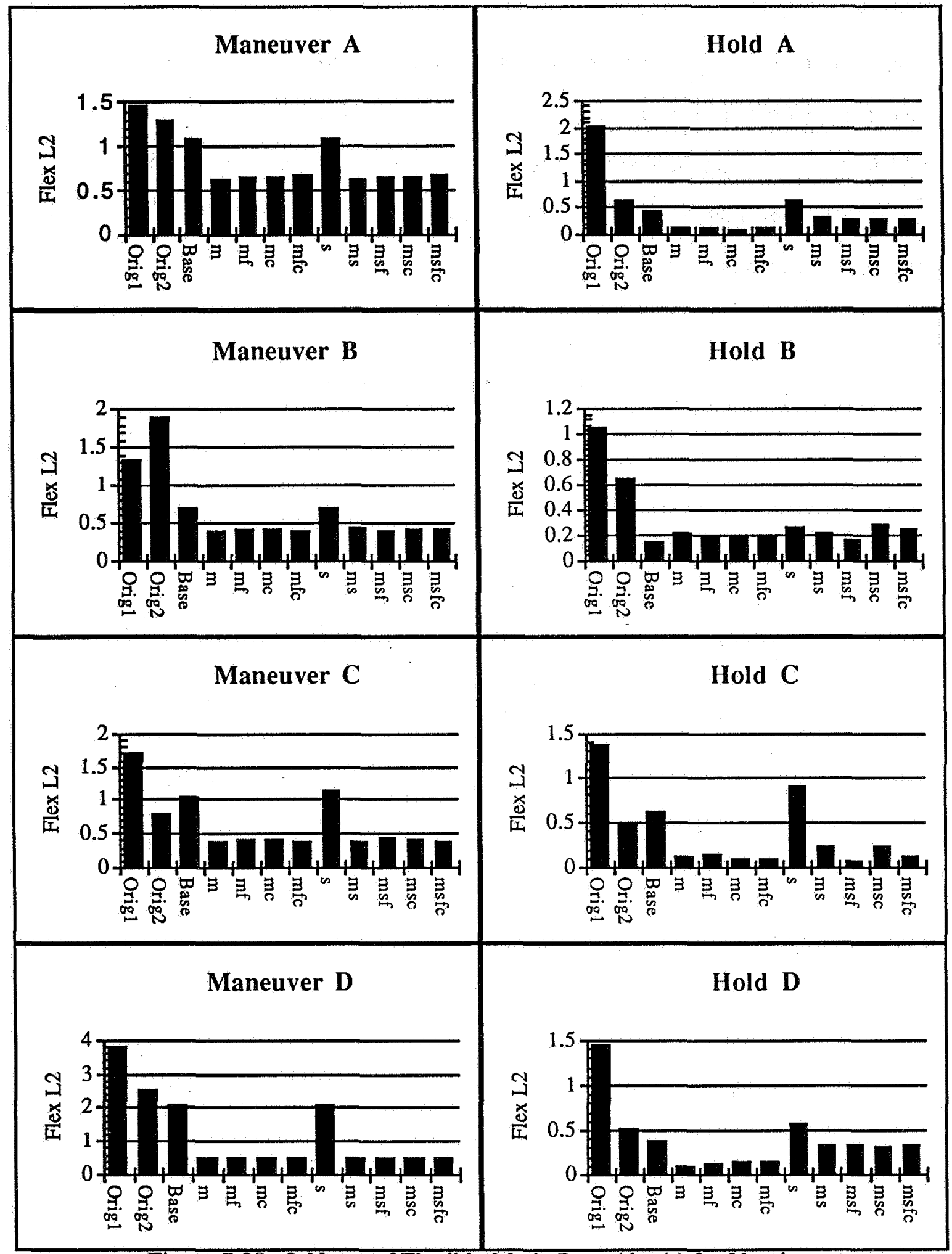

Figure 7.39. 2-Norm of Flexible Mode Rates(deg/s) for Verniers 


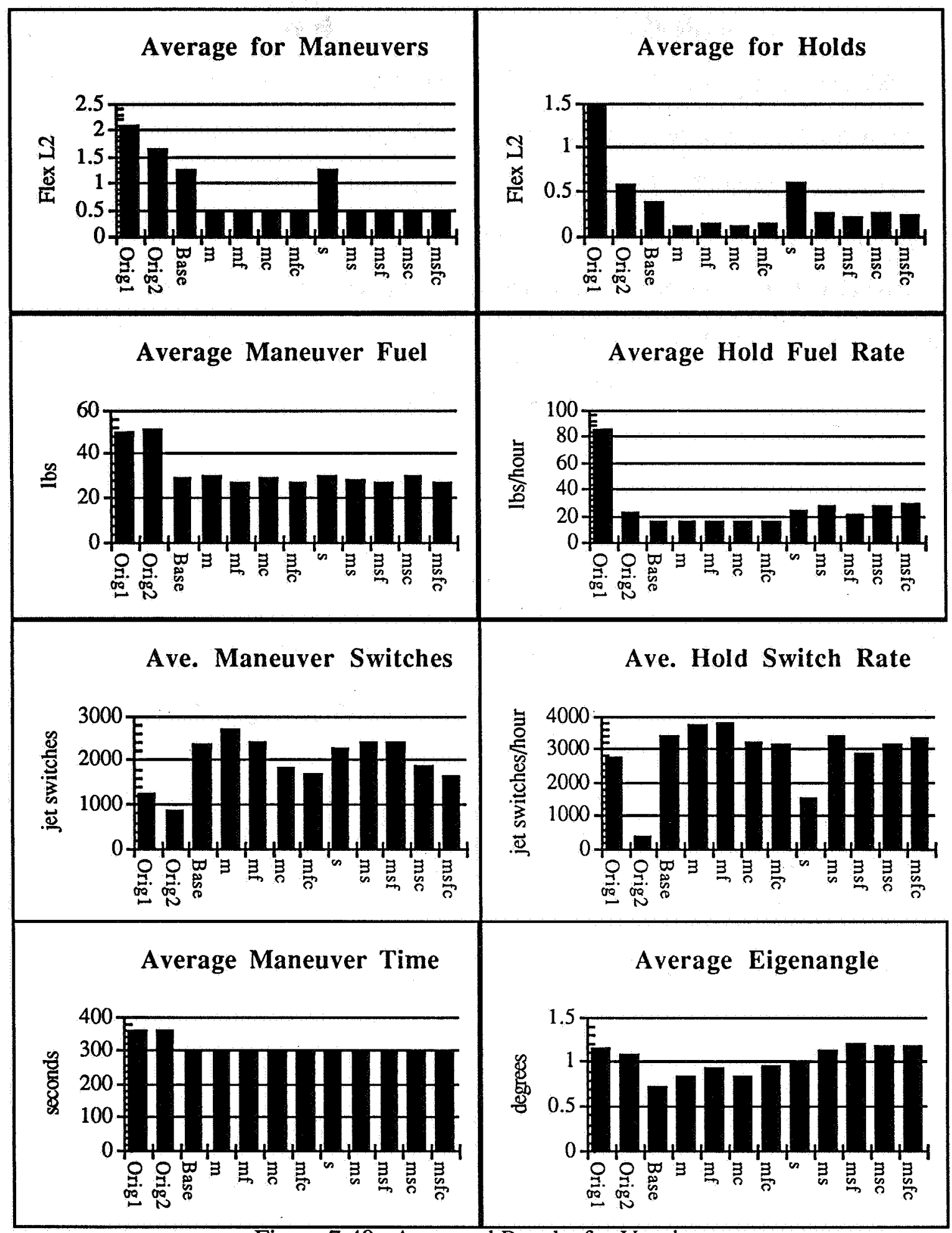

Figure 7.40. Averaged Results for Verniers 
The similar maneuver completion times and attitude hold eigenangles, Figure 7.40 , indicate that fair comparisons of fuel consumption and jet switches have been achieved. The fuzzy controllers all consumed about half the amount of fuel required by the FCS controllers to complete a maneuver. The fuzzy approach which does not specify a maneuver attitude trajectory and reduces corrective firings during the maneuver coast phase becomes more beneficial in the presence of flexibility than in the rigid case since fewer firings translate into less excitation of the flexible modes. Using the anti-flex rule package definitely leads to more jet switching. During attitude hold, turning on the package doubles the jet switch rate compared to cases with it off. This is due to the fuzzy attitude controller adjusting its jet selection more during a burn in response to the flexible modes than it would normally.

The following figures show time histories from Maneuver D. Maneuver D causes the greatest excitation of the flexible modes, as expected since it is primarily a maneuver in roll. The first thing to note is the difference between the pitch and yaw rates for the FCS and for the fuzzy attitude controller. The fuzzy attitude controller selects jets which avoid exciting the modes which couple into the yaw and pitch axes. The FCS excites them, especially in yaw. It is inevitable that flexible modes coupling into roll will be excited since a large rate change in roll is required for this maneuver; however, the fuzzy attitude controller manages to damp these modes where the FCS excites them continuously. The penalty of course is increased jet switches.

The fuzzy attitude controller attains a nice one sided limit cycle based upon the disturbance acceleration estimate. Fuel use tapers off at this point. The FCS does much worse in this respect. It does not settle into a nice firing pattern until about 1500 seconds. This may be due in part to insufficient attenuation of the flexible dynamics by the FCS disturbance acceleration filter. 

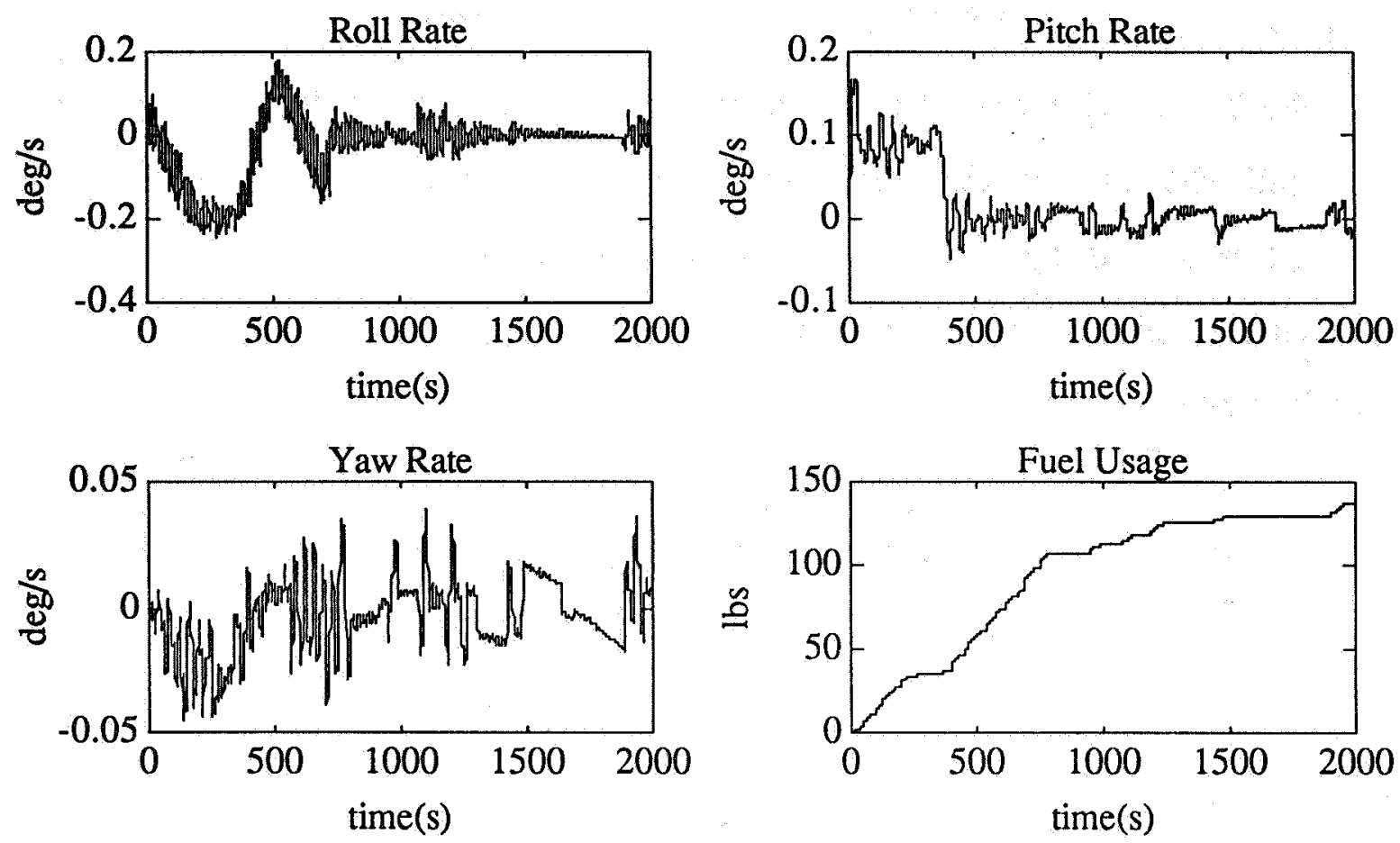

Figure 7.41. Body Rates and Fuel Usage for Verniers, Maneuver D, FCS 'Orig1'
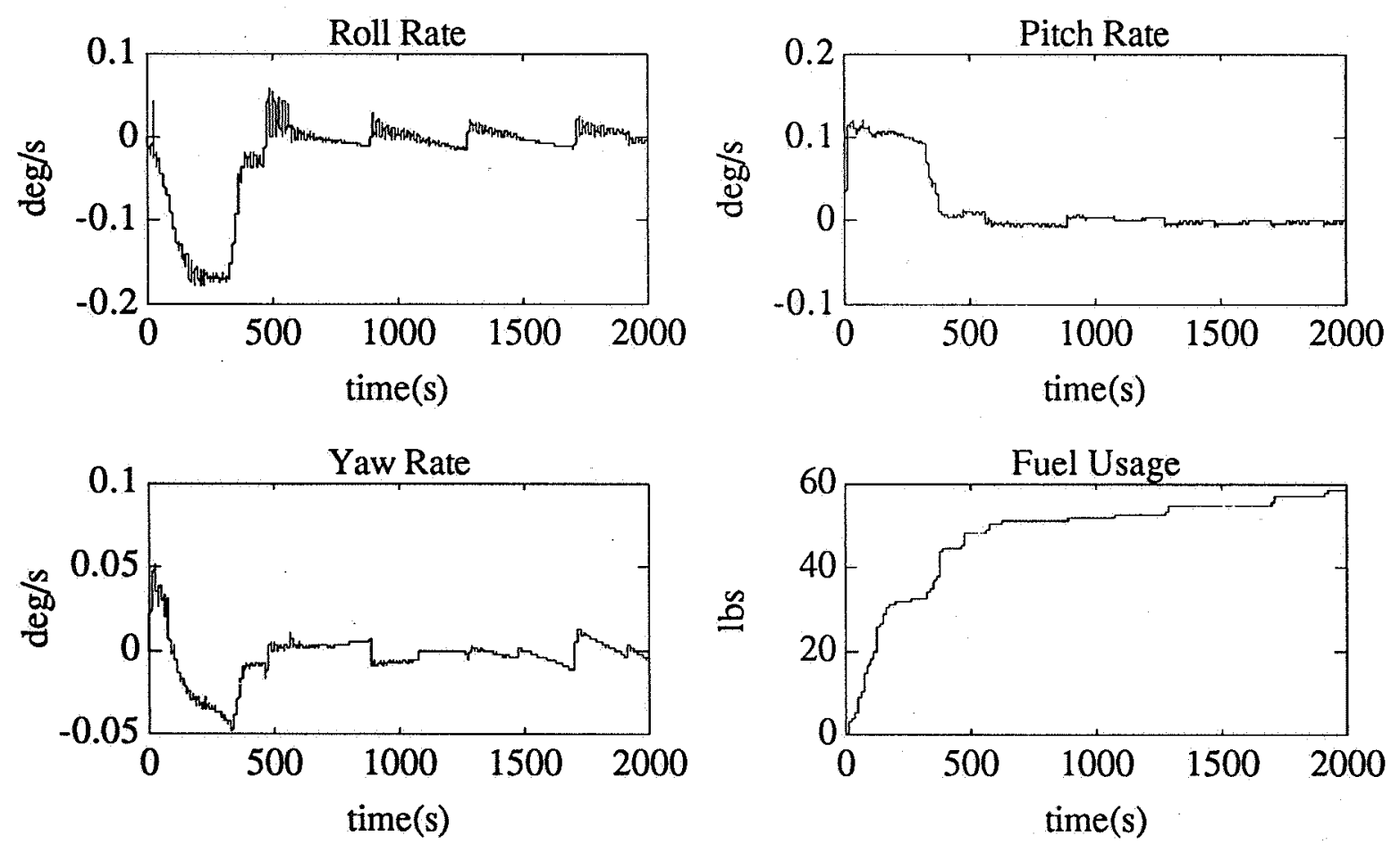

Figure 7.42. Body Rates and Fuel Usage for Verniers, Maneuver D, Fuzzy Attitude Controller ' $\mathrm{msfc}$ ' 
Figure 7.43 and Figure 7.44 show the results for Alt Mode. The comparative reduction in the flex 2-norm achieved by the fuzzy controller is not as great as that for the verniers, but it is still reduced by about a factor of two on average. This reduction is performed while using less fuel and requiring fewer jet switches than either of the FCS runs. As was the case for the rigid maneuvers, the anti-chatter and jet limiting rules cause generally worse performance.

In comparing the run with only the slow trajectory rules active to the run which added the anti-flex rules, the cost of reducing the flex 2-norm becomes apparent, Figure 7.44. Maneuver fuel was not affected much but there was a significant rise in the attitude hold fuel consumption rate due to the active damping of flexible modes after what would typically have only been a single jet pulse. Correspondingly, the jet switch rate is also higher.

The Figures 7.45 and 7.46 show the body rates from maneuver D for the FCS, configuration one, and the fuzzy attitude controller using the slow disturbance and anti-flex rule packages. The same things that were said about the vernier runs can be said about these. The FCS does do a better job of achieving longer limit cycles in this case, although it performs rate changes in both directions. The fuel plots show that the fuzzy attitude controller required about half the fuel used by the FCS to complete the maneuver and actively damp the flexible modes. 


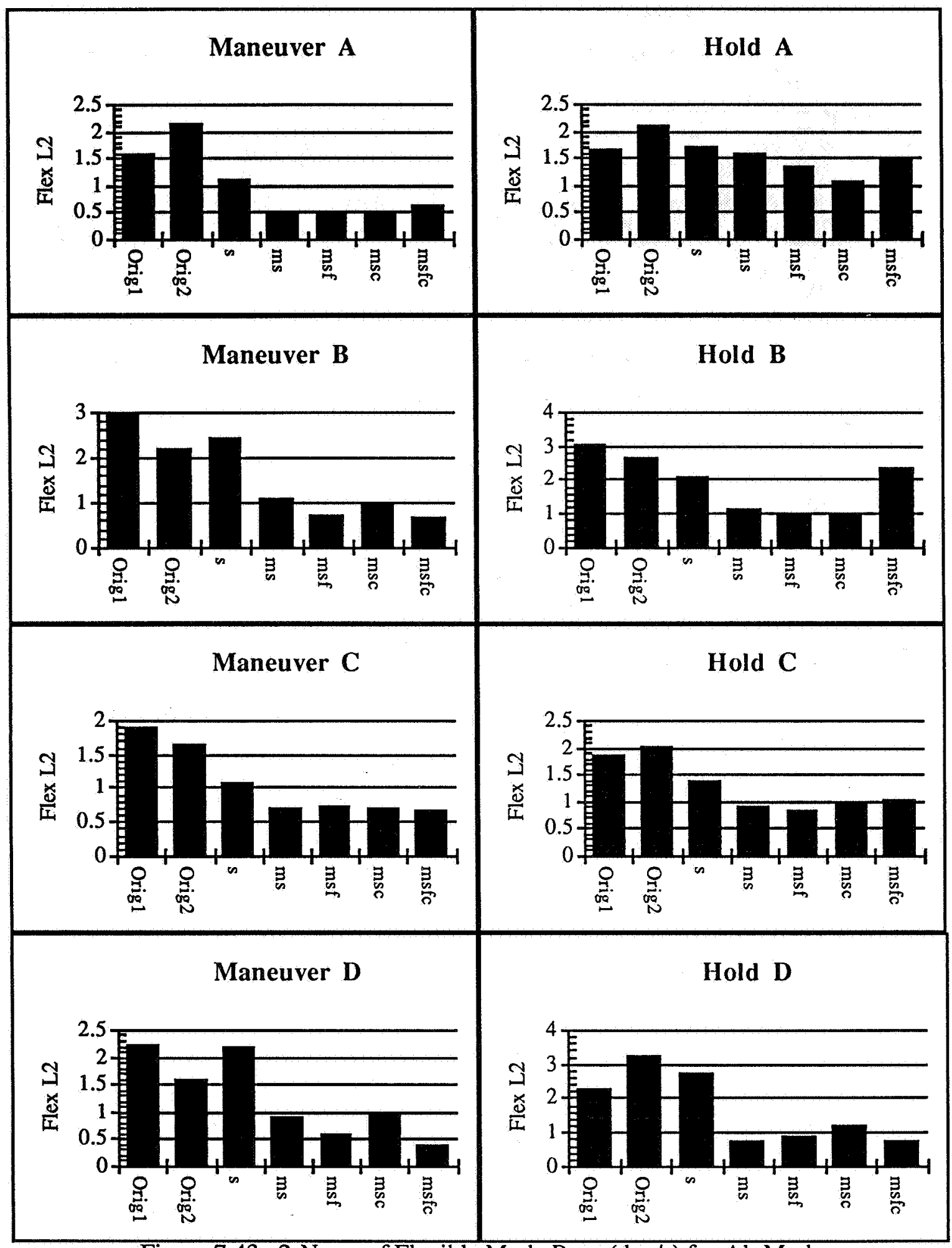

Figure 7.43. 2-Norm of Flexible Mode Rates(deg/s) for Alt Mode 


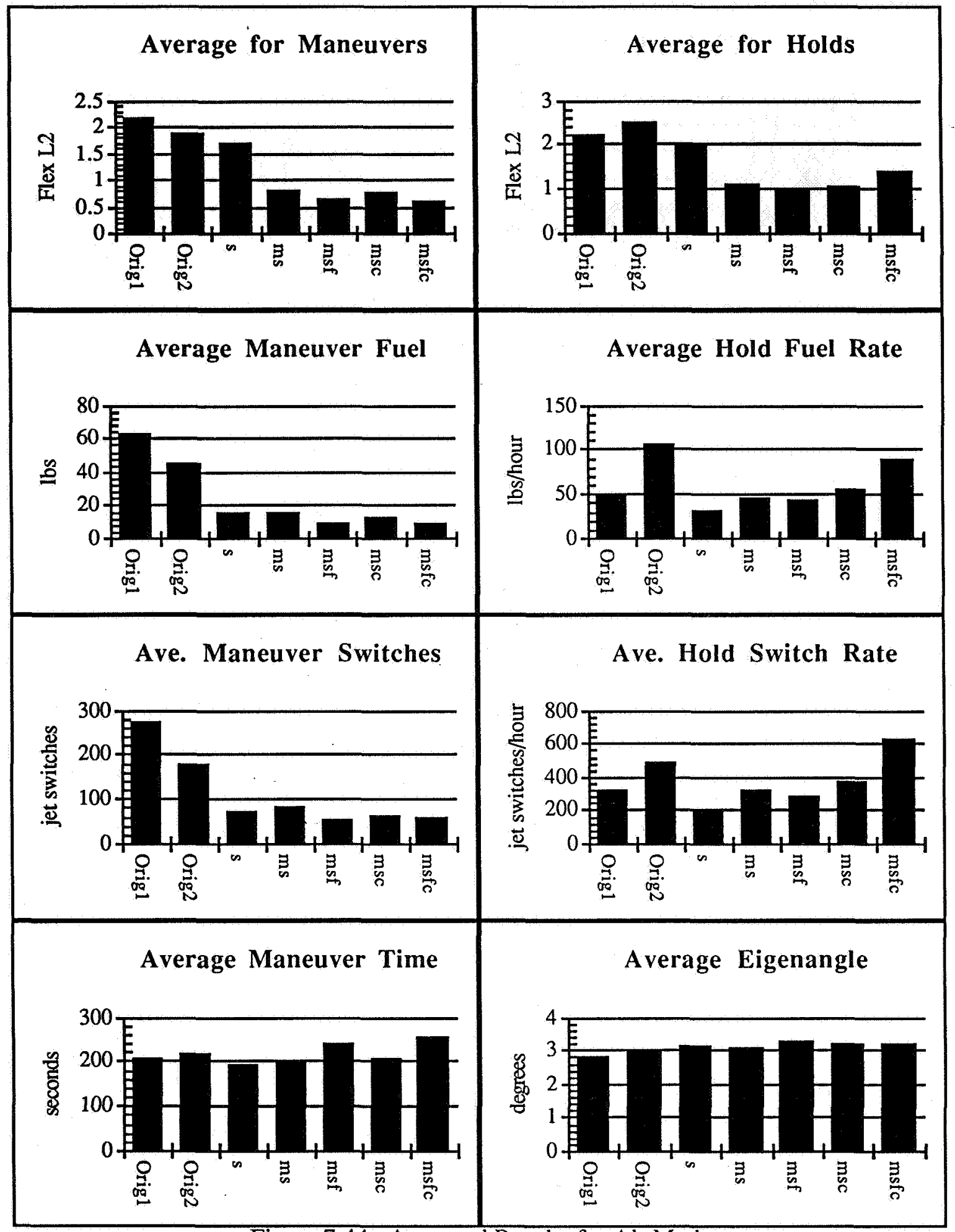

Figure 7.44. Averaged Results for Alt Mode 

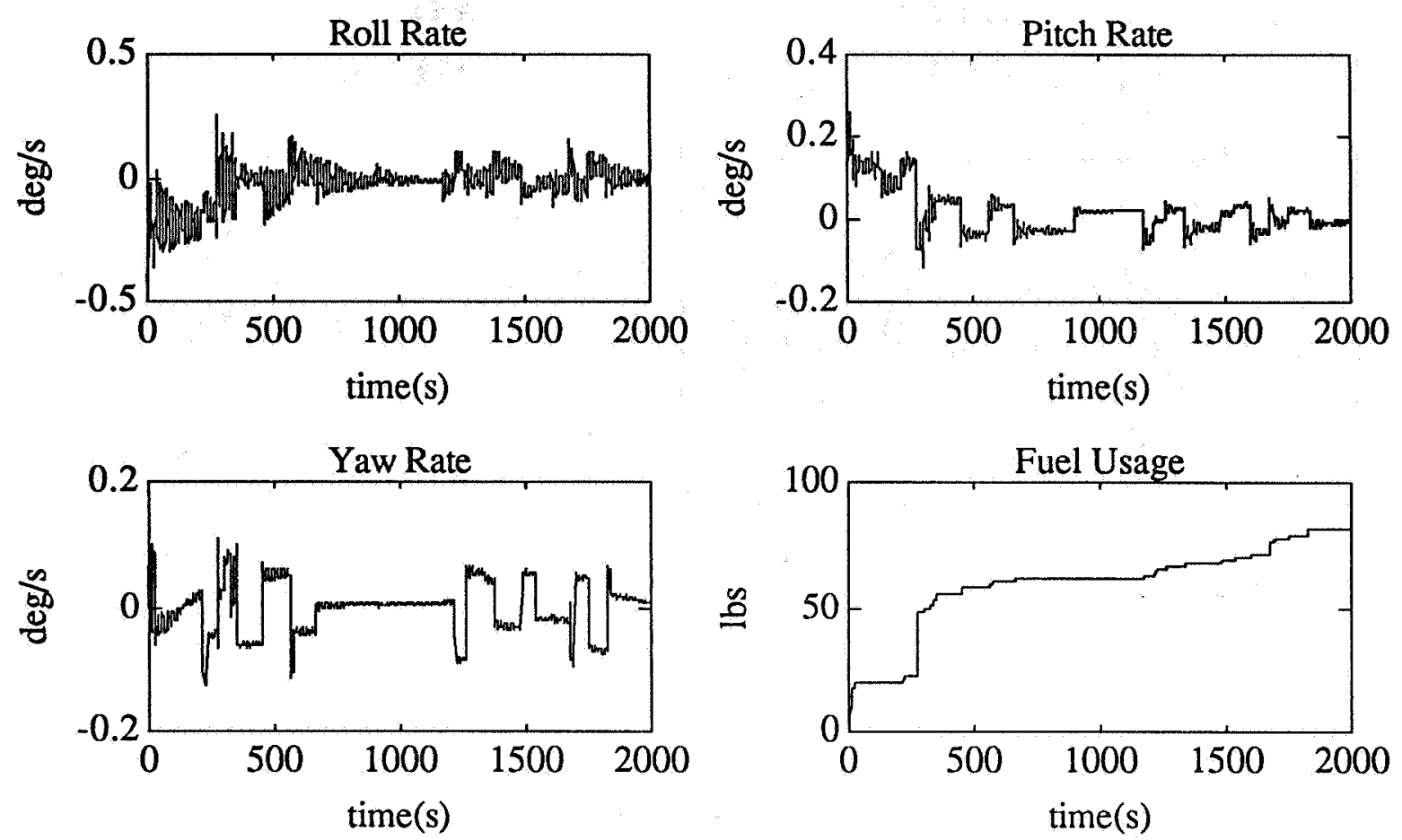

Figure 7.45. Body Rates and Fuel Usage for Alt Mode, Maneuver D, FCS 'Orig1'
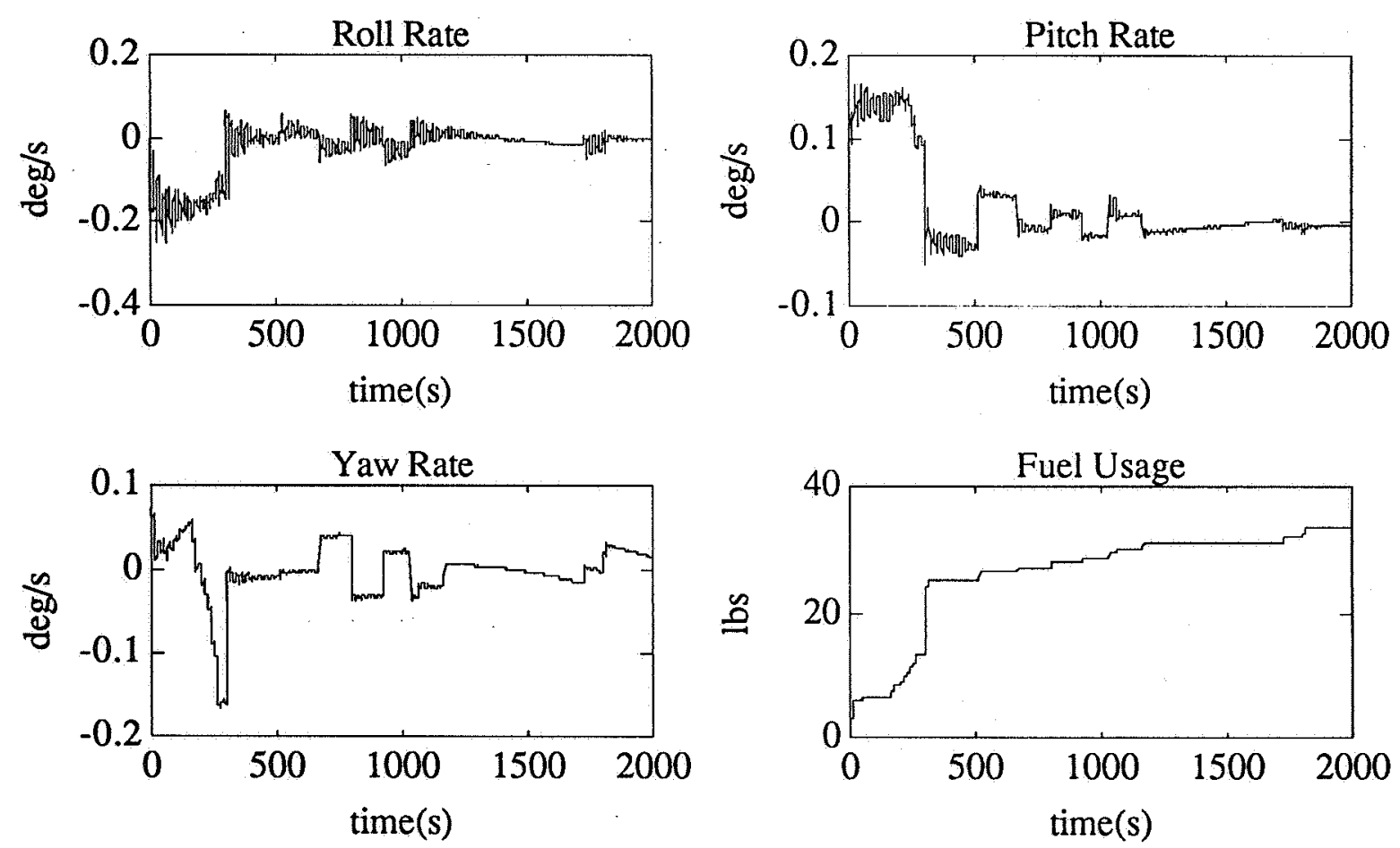

Figure 7.46. Body Rates and Fuel Usage for Alt Mode, Maneuver D, Fuzzy Attitude Controller ' $m s^{\prime}$ 


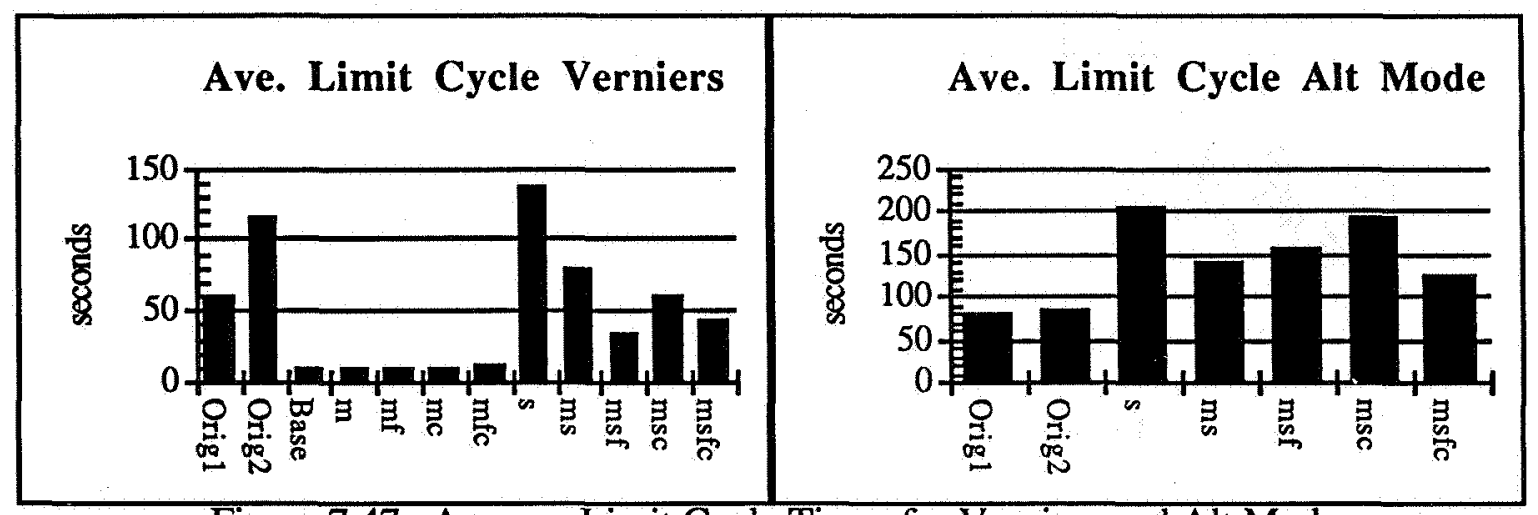

Figure 7.47. Average Limit Cycle Times for Verniers and Alt Mode

The length of time spent coasting on the slow disturbance trajectory is important because enough time must transpire for the transients from the last jet firings to die out of the disturbance acceleration estimate. According to Figure 7.47, when operating on verniers, limit cycles often were not long enough to allow for an accurate disturbance acceleration estimate forcing the controller to ignore the estimate and just command a rate change that points at the center of the error envelope as the baseline rules do. This suggests that jet switching performance for verniers, which is related to the length of the limit cycles, could be improved if a better filtering technique could be established, providing accurate information about the disturbance acceleration more rapidly.

\subsection{Off Nominal Results}

It has been previously stated that a controller based on fuzzy sets and logic is more flexible and adaptive than one based on crisp boundaries. To demonstrate this the robustness of both the FCS and fuzzy attitude controller to changes in operating specifications are discussed for both the rigid and flex cases. In the flex cases, flex modeling error is examined. Previous results illustrated fair comparisons between the FCS and fuzzy controllers by conducting maneuvers and attitude holds to mimic usual space shuttle operations. Here the question is asked, what happens if the attitude controllers are asked to operate in regimes which are not usually encountered during space shuttle operations? 
First, to study the affects of diverting from typical space shuttle operating conditions, the pointing requirements were tightened. For verniers the FCS deadband was reduced by a factor of four to .25 degrees. The pointing error constraint for the fuzzy attitude controller was set to .43 degrees to achieve similar pointing accuracy as reflected by the average eigenangle during attitude hold. The Alt Mode deadband was reduced by a factor of three to 1 degree. The corresponding fuzzy pointing error constraint was set at 1.42 degrees to achieve the same average eigenangle during attitude hold.

Both the FCS and the fuzzy attitude controller require knowledge of the flexible dynamics in order to perform their mission in its presence. To determine the effect of flexible modeling errors, the flexible dynamics used during simulation were modified. All modes were decreased in frequency by $20 \%$. Modal amplitudes were increased by $20 \%$. Runs were also conducted with the modal frequencies increased by $20 \%$.

\subsubsection{Orbiter Alone}

For orbiter alone mass properties maneuver $\mathrm{A}$ was chosen for the vernier jet runs with the tightened pointing requirements. The FCS was compared to the 'sfc' fuzzy attitude controller. Maneuver C was used for Alt Mode with the FCS compared to the fuzzy attitude controller with only slow trajectory rules active. The results are presented first for verniers (Figures 7.48 through 7.50) and then for Alt Mode (Figures 7.51 through 7.53). 

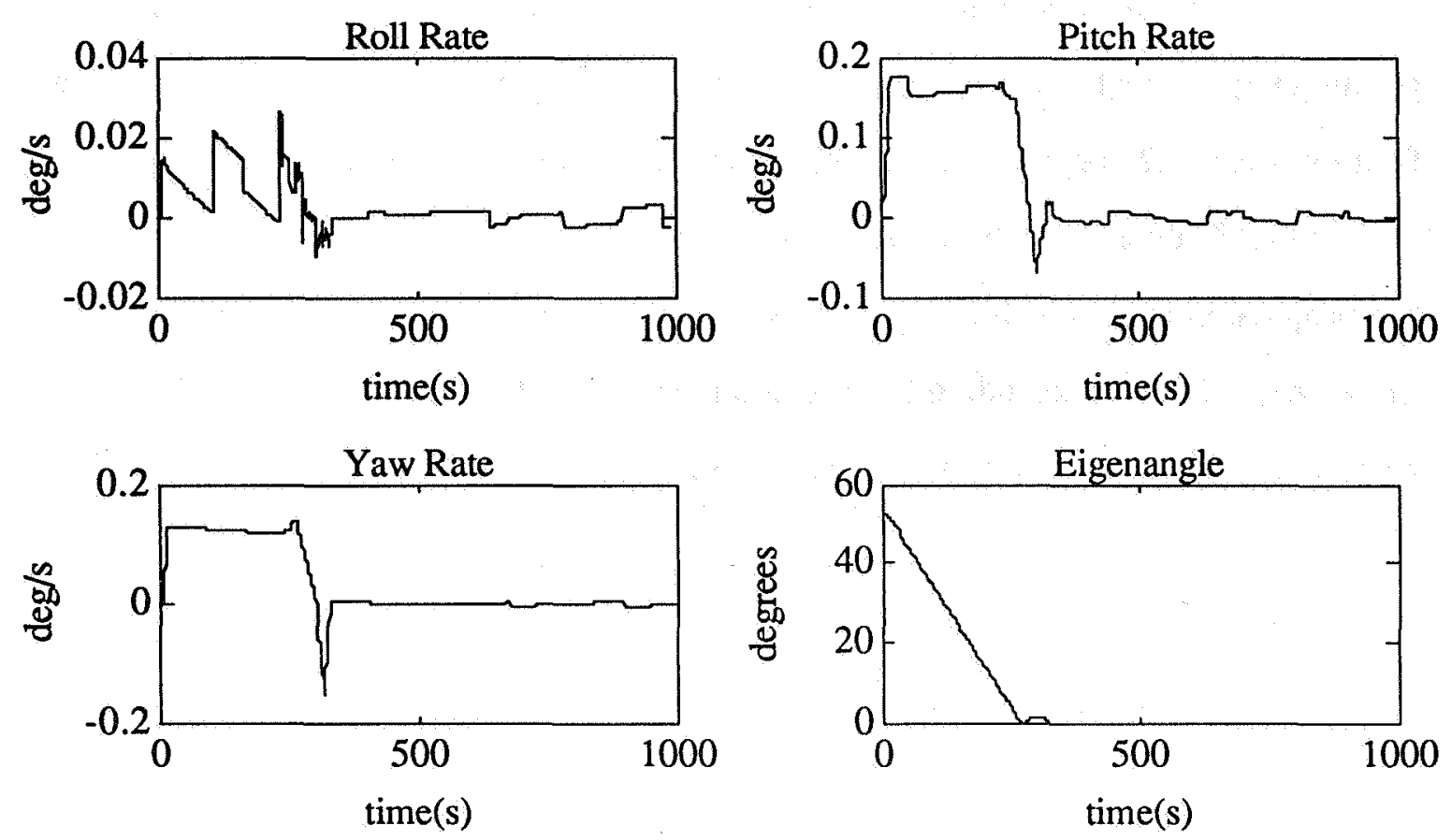

Figure 7.48. FCS Body Rates and Eigenangle for Maneuver A using Verniers
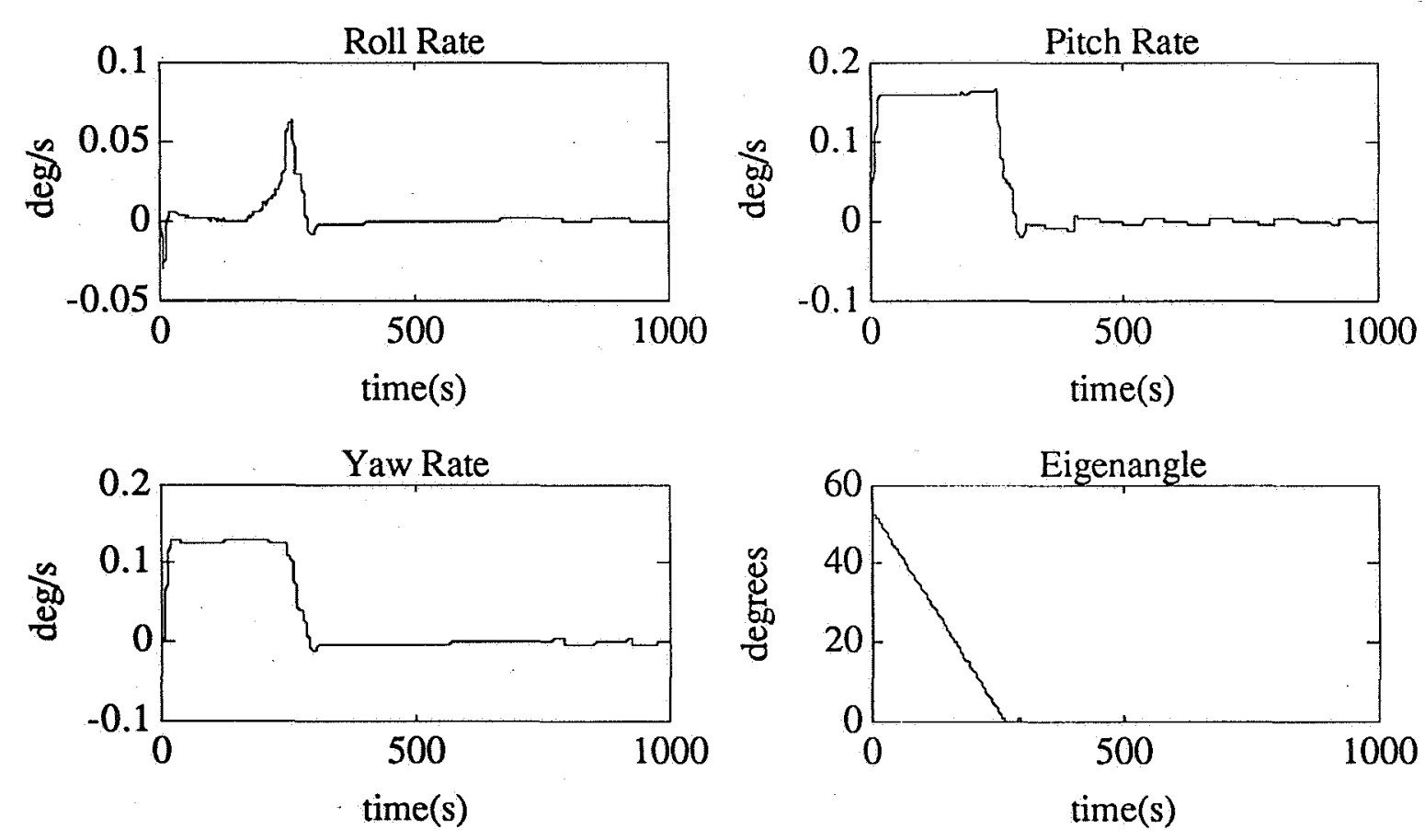

Figure 7.49. Fuzzy Attitude Controller Body Rates and Eigenangle for Maneuver A using Verniers 

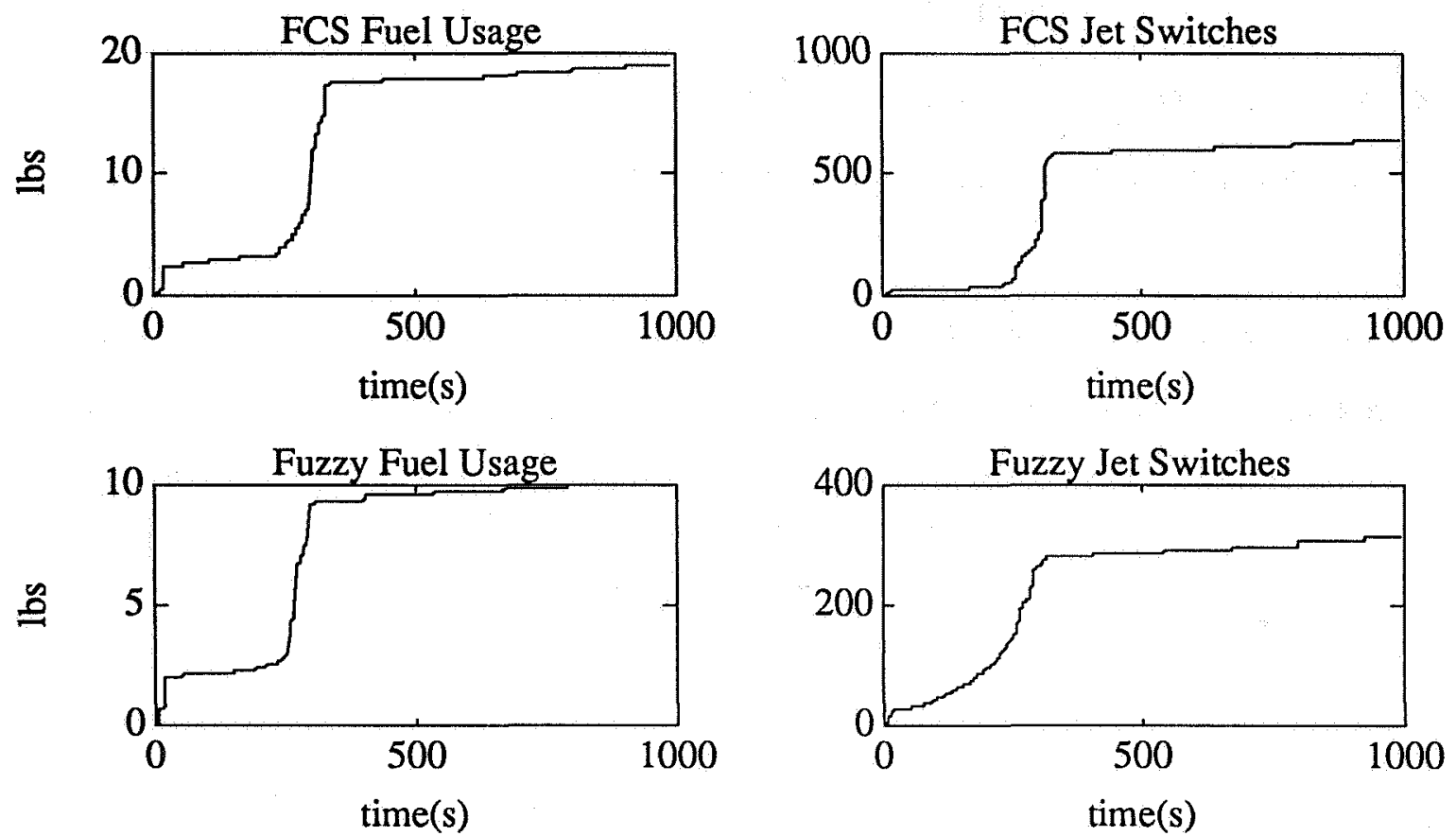

Figure 7.50. Comparison of Fuel Consumption and Jet Switches for Maneuver A using Verniers

Tightening the pointing requirements causes the FCS, which previously outperformed the fuzzy attitude controller, to have several problems. The result is that the fuzzy attitude controller whose performance was less sensitive to the pointing requirements now uses less fuel and jet switches. Surprisingly, the fuzzy attitude controller actually uses slightly less fuel than it did with nominal pointing, Table 7.8.

The first FCS performance problem to note is the poor maneuver completion. The miss of the desired final attitude is visible as the hump in the eigenangle plot, Figure 7.49. The underlying flaw with the FCS which causes this problem is its reliance upon crisp switching curves. The FCS can not perform a maneuver deceleration with enough accuracy to end up inside the off nominal deadband which is much smaller than in the nominal case. It then either commences another maneuver if twice (a crisp boundary) the attitude deadband is exceeded or fires until it hits the drift channel, see section 6.5. Either way this leads to the second problem. When the deceleration trajectory does not enter the 
deadband, too much rate will be commanded in the opposite direction. These large rate changes are especially visible in the yaw rate time history. The result is that the FCS uses about twice as much fuel to complete the maneuver.

The fuzzy attitude controller has no crisp deadband which it must acquire at the end of a deceleration burn in order to prevent the large rate change problem described above. It does not use crisp boundaries to determine whether the rate should be zero or the maneuver rate. Rather there is a smooth transition from commanding zero rate to the maneuver rate based upon how big the attitude error has become. There is no point at which the attitude error will cause the commanded rate to jump from zero to the maneuver rate. Table 7.8 documents the change in performance when changing from the nominal to off nominal pointing requirements.

Table 7.8. Comparison of Nominal to Off Nominal Results for the FCS and Fuzzy Attitude Controllers using Verniers

\begin{tabular}{||c|c|c|c|c||}
\hline & Nominal FCS & $\begin{array}{c}\text { Off Nominal } \\
\text { FCS }\end{array}$ & $\begin{array}{c}\text { Nominal } \\
\text { Fuzzy }\end{array}$ & $\begin{array}{c}\text { Off Nominal } \\
\text { Fuzzy }\end{array}$ \\
\hline $\begin{array}{c}\text { Maneuver } \\
\text { Fuel(lbs) }\end{array}$ & 9.0 & 17.6 & 11.2 & 9.3 \\
\hline $\begin{array}{c}\text { Maneuver Jet } \\
\text { Switches }\end{array}$ & 212 & 588 & 252 & 276 \\
\hline $\begin{array}{c}\text { Hold Fuel } \\
\text { Rate(lbs/hour) }\end{array}$ & 6.57 & 7.3 & 3.83 & 3.4 \\
\hline $\begin{array}{c}\text { Hold Jet } \\
\text { Switch Rate } \\
\text { (switches/hour) }\end{array}$ & 134.9 & 293.1 & 168.3 & 176.4 \\
\hline $\begin{array}{c}\text { Average Hold } \\
\text { Eigenangle(deg) }\end{array}$ & 1.18 & .27 & 1.02 & .28 \\
\hline
\end{tabular}

The following figures present results from the Alt Mode case. As for verniers, the tighter pointing requirements cause the FCS similar problems. 

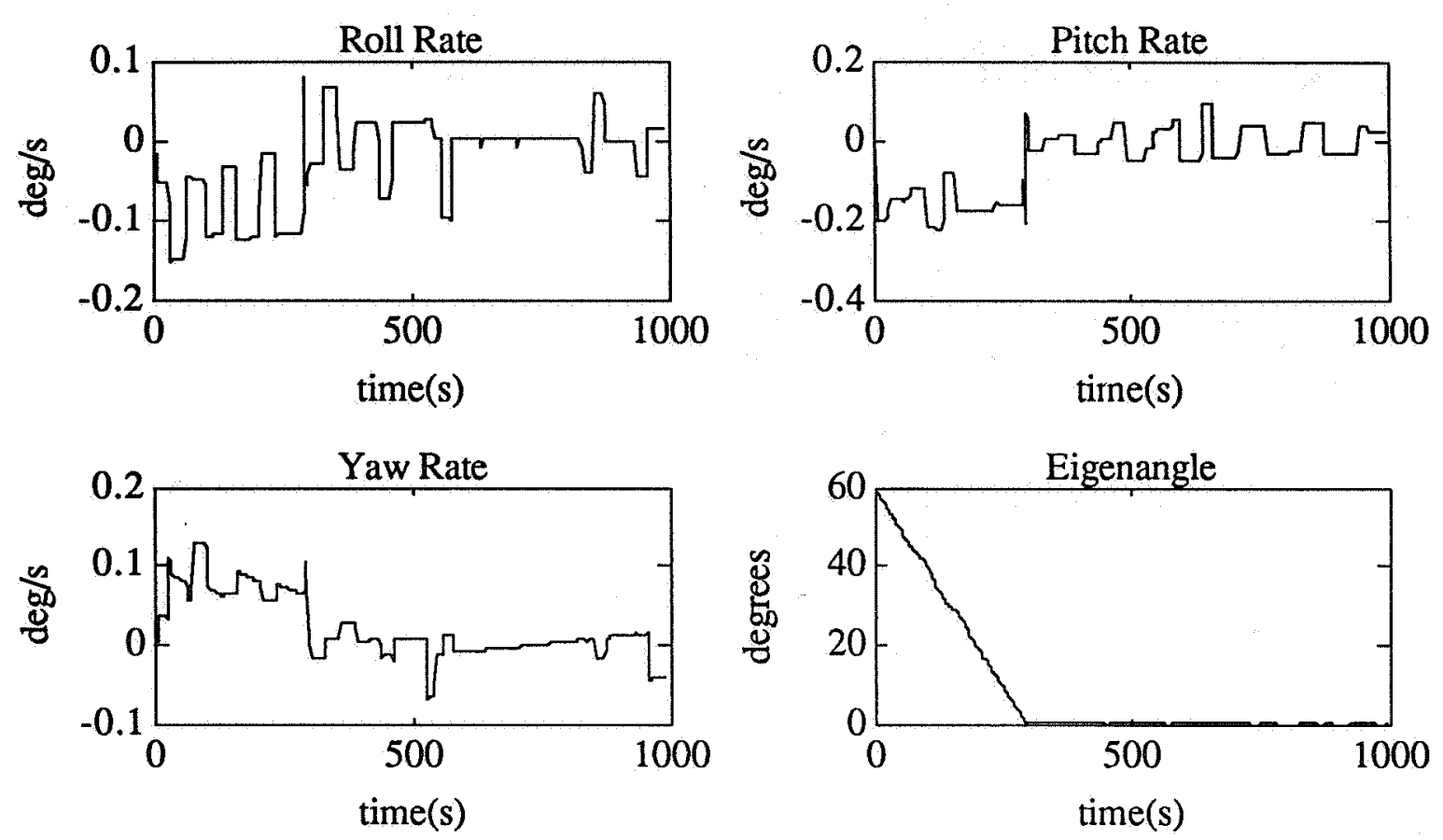

Figure 7.51. FCS Body Rates and Eigenangle for Maneuver C using Alt Mode
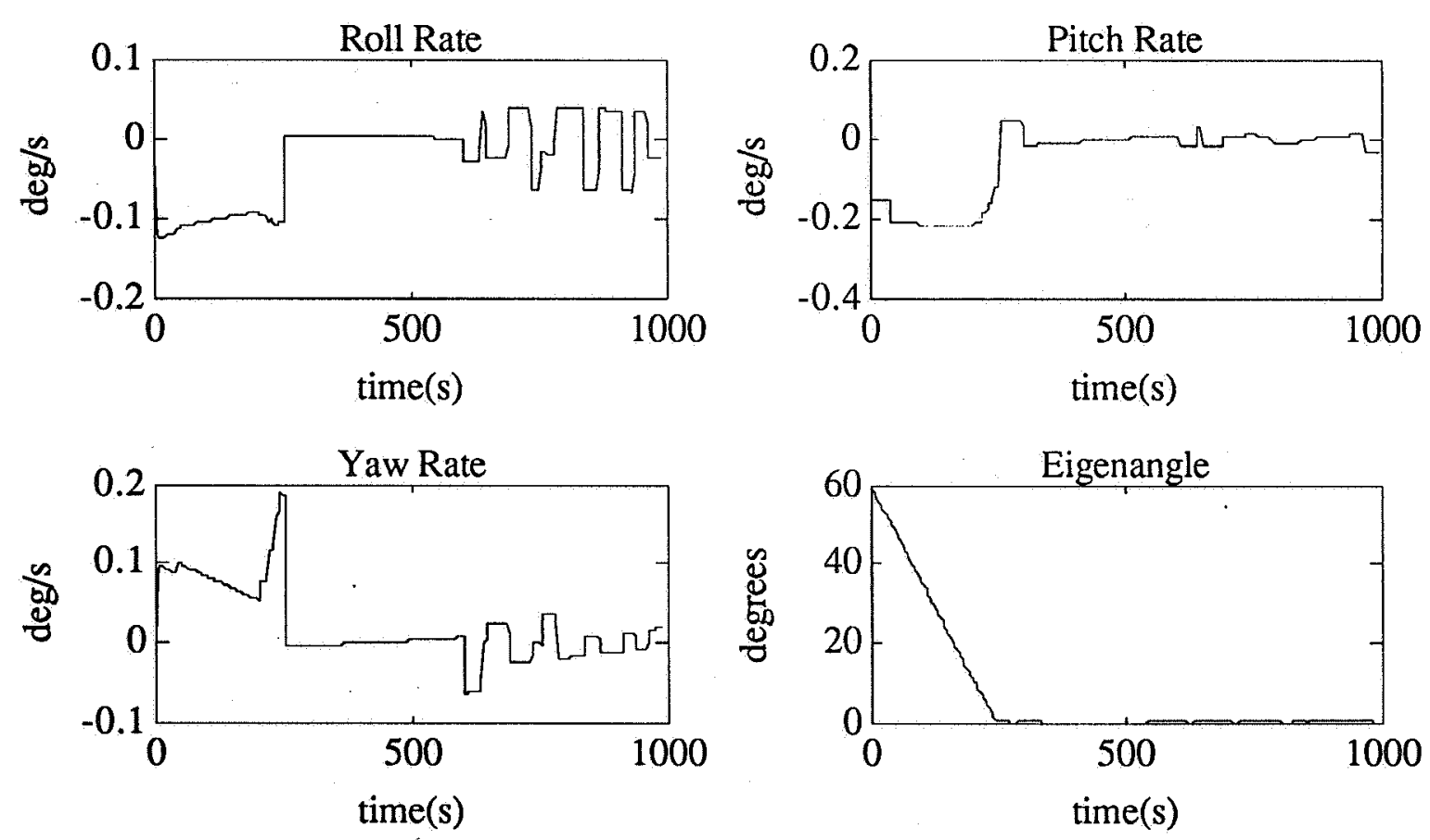

Figure 7.52. Fuzzy Attitude Controller Body Rates and Eigenangle for Maneuver $\mathrm{C}$ using Alt Mode 

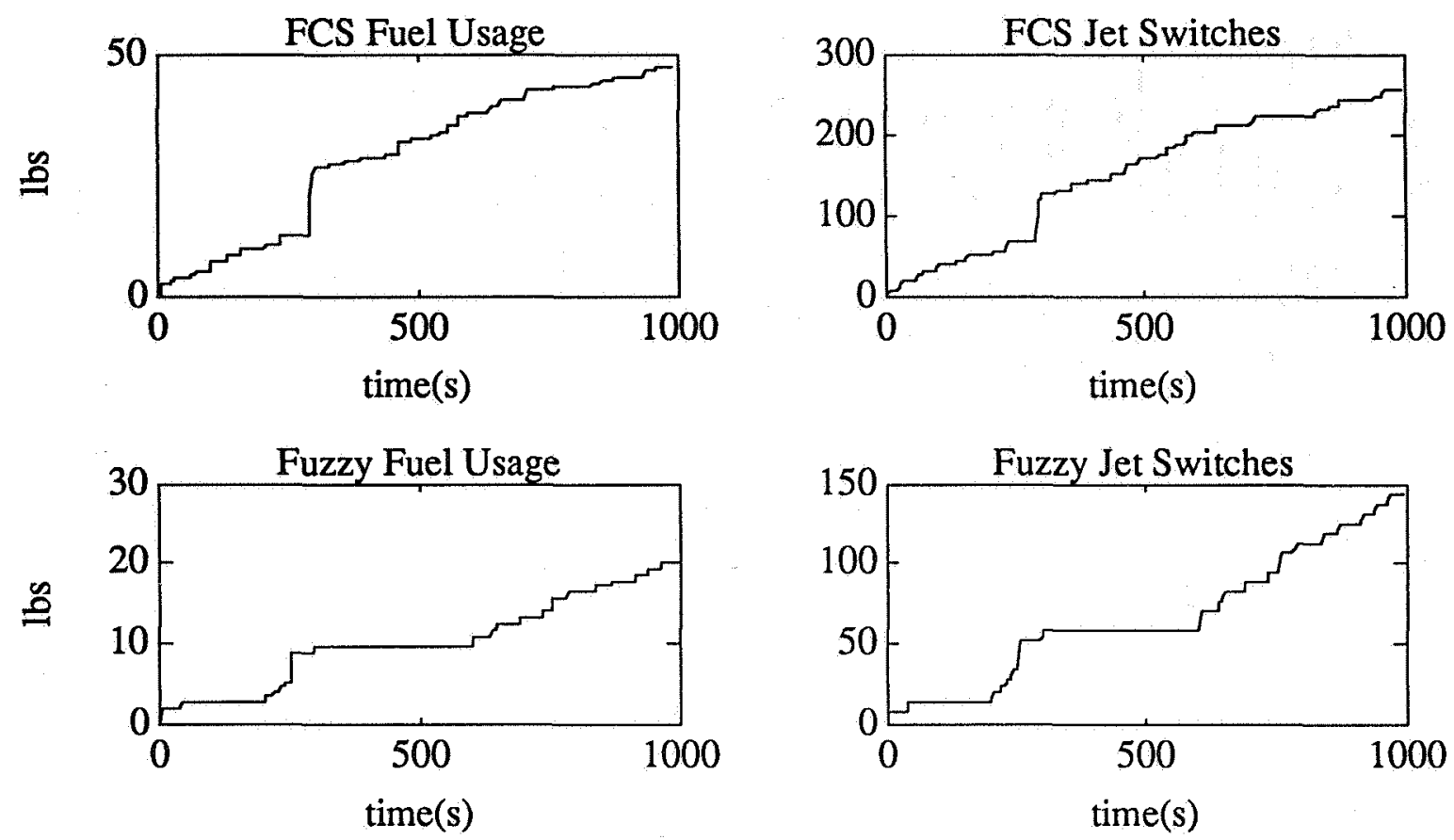

Figure 7.53. Comparison of Fuel Consumption and Jet Switches for Maneuver $\mathrm{C}$ using Alt Mode

As described earlier the FCS prescribes an attitude trajectory to be followed throughout a maneuver. With the smaller deadbands the excess rate changes this can cause is apparent. The large rate changes in roll, Figure 7.51 , bear testimony to this. Ultimately this leads to about twice as much fuel being consumed as for the fuzzy attitude controller, Figure 7.53. The fuzzy attitude controller whose rules do not specify any trajectory through which the error should be removed avoids all the limit cycling during the coast phase of the maneuver.

Neither controller performs exceptionally during the attitude hold. The small deadband does not provide a large enough area for setting up a one sided limit cycle. The disturbances are small enough such that even a rate change of the minimum impulse directly opposite the disturbance acceleration will not be reversed before the allowable error is excursed. 
Table 7.9 compares previous results with the current ones. As expected the FCS maneuver fuel performance is quite sensitive to the pointing requirements. The FCS experienced a $78 \%$ increase in fuel consumption while the fuzzy attitude controller required only $21 \%$ more fuel. For the other results the FCS had a greater percentage increase than the fuzzy attitude controller, but not to the extent that maneuver fuel consumption did.

Table 7.9. Comparison of Nominal to Off Nominal Results for the FCS and Fuzzy Attitude Controllers using Alt Mode

\begin{tabular}{|c|c|c|c|c||}
\hline & Nominal FCS & Off Nominal & $\begin{array}{c}\text { Nominal } \\
\text { F CS }\end{array}$ & $\begin{array}{c}\text { Off Nominal } \\
\text { Fuzzy }\end{array}$ \\
\hline $\begin{array}{c}\text { Maneuver } \\
\text { Fuel(lbs) }\end{array}$ & 14.9 & 26.5 & 7.3 & 8.8 \\
\hline $\begin{array}{c}\text { Maneuver Jet } \\
\text { Switches }\end{array}$ & 74 & 126 & 38 & 52 \\
\hline $\begin{array}{c}\text { Hold Fuel } \\
\text { Rate(lbs/hour) }\end{array}$ & 34.8 & 106.5 & 23.2 & 55.0 \\
\hline $\begin{array}{c}\text { Hold Jet } \\
\begin{array}{c}\text { Switch Rate } \\
\text { (switches/hour) }\end{array}\end{array}$ & 238.3 & 658.2 & 173.1 & 433.7 \\
\hline $\begin{array}{c}\text { Average Hold } \\
\text { Eigenangle(deg) }\end{array}$ & 2.97 & .97 & 3.01 & .85 \\
\hline
\end{tabular}

\subsubsection{Orbiter with GRO, Modeled as Rigid}

The same maneuvers, controllers, and pointing error requirements were used to generate results for the orbiter-GRO mass properties. Again results from runs using Vernier jet are presented followed by the Alt Mode results.

Although the rate plots look fairly reasonable for the FCS vernier case, Figure 7.54, pitch and yaw rate ramp up to the required rate for the maneuver and the maneuver deceleration is slightly more accurate than the orbiter alone case, but the fuel and jet switches plots tell a different story. The FCS is firing jets almost continuously throughout the entire maneuver. There is no leveling off of fuel consumption which is normally seen during the middle of a maneuver. 

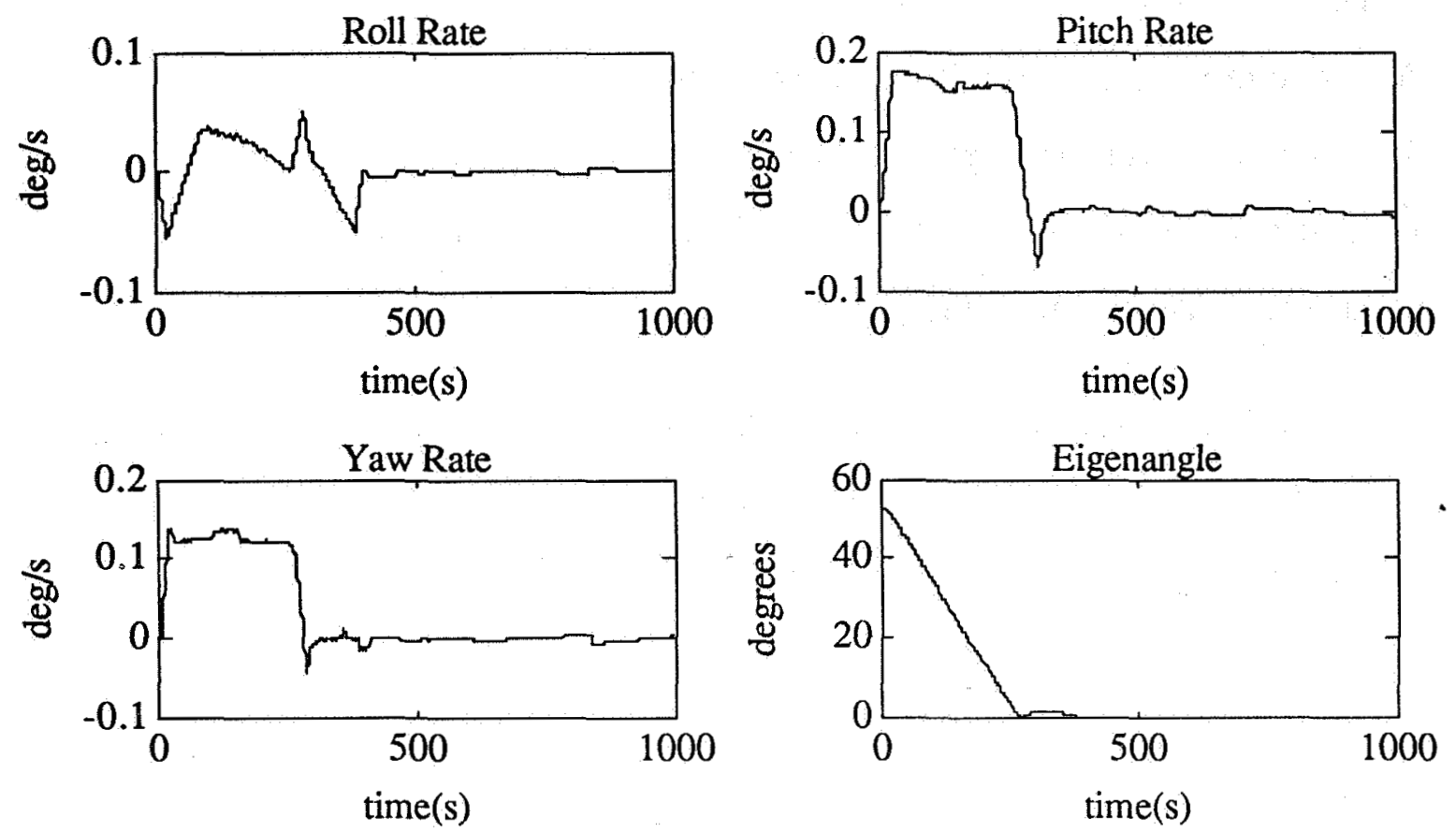

Figure 7.54. FCS Body Rates and Eigen Angle for Maneuver A using Verniers
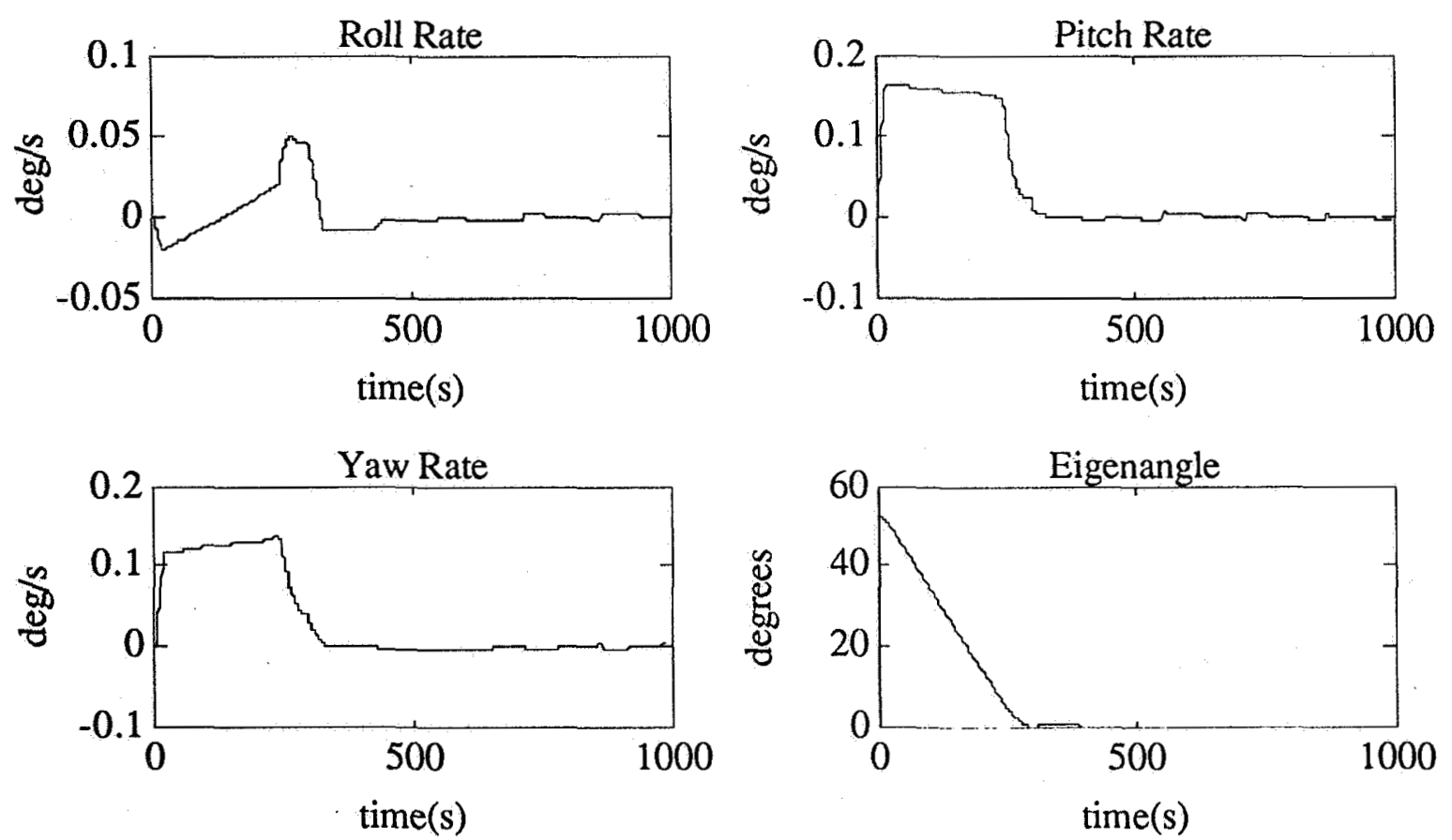

Figure 7.55. Fuzzy Attitude Controller Body Rates and Eigen Angle for Maneuver A using Verniers 

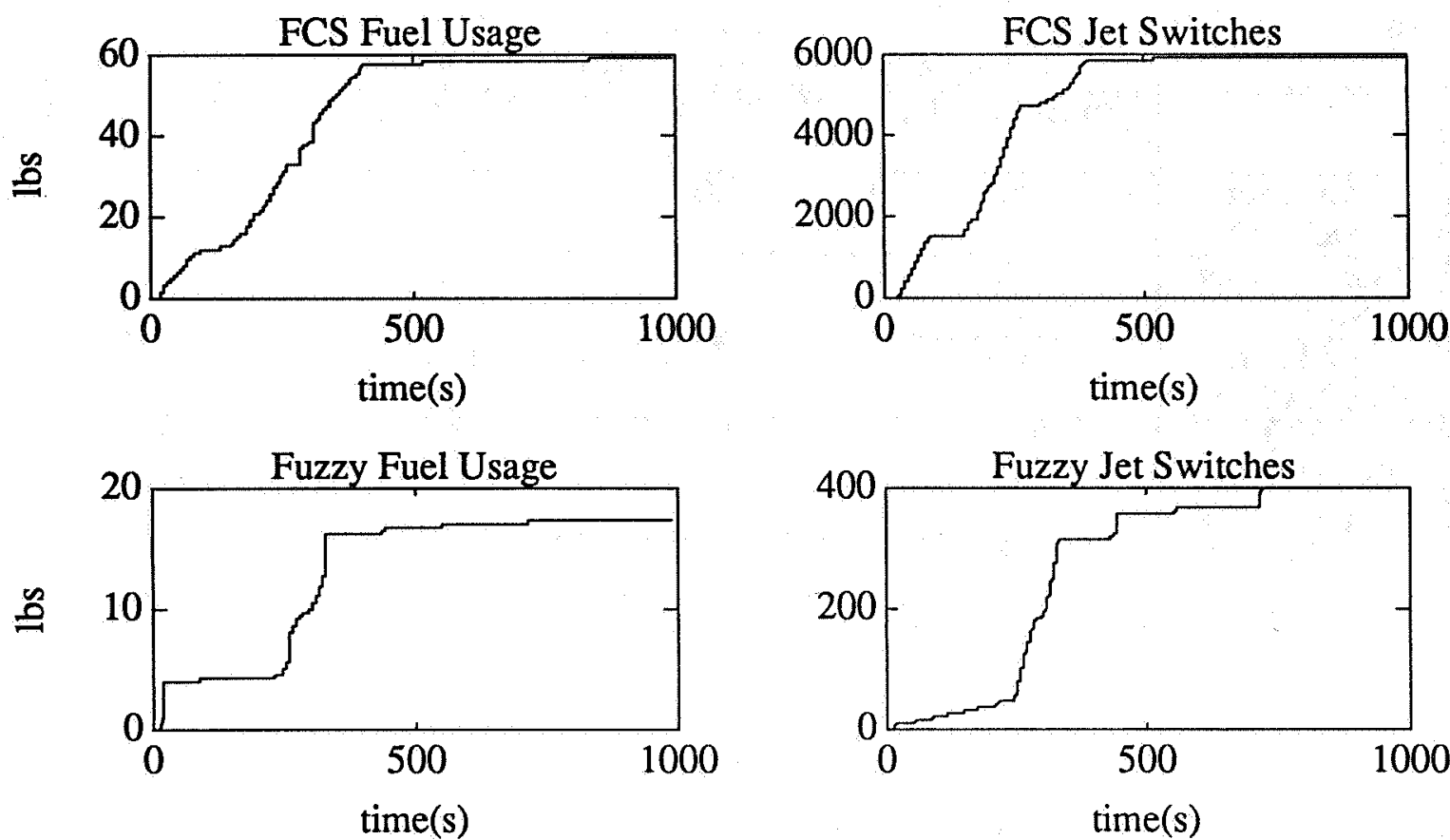

Figure 7.56. Comparison of Fuel Consumption and Jet Switches for Maneuver A using Verniers

The problem is caused by the low control authority in the roll axis due to the combined mass properties. Even though very little roll is required for maneuver A, the FCS performs rapid rate changes in roll which require a lot of firings.

Once again, as table 7.10 indicates, performance of the fuzzy attitude controller actually improves slightly with the tighter pointing requirements. This is unexpected and not readily explained. The jet switching rate for the FCS soars much above anything encountered previously. 
Table 7.10. Comparison of Nominal to Off Nominal results with Rigid-GRO for the FCS and Fuzzy Attitude Controllers using Verniers

\begin{tabular}{|c|c|c|c|c||}
\hline & Nominal FCS & Off Nominal & $\begin{array}{c}\text { Nominal } \\
\text { F C S }\end{array}$ & $\begin{array}{c}\text { Off Nominal } \\
\text { Fuzzy }\end{array}$ \\
\hline $\begin{array}{c}\text { Maneuver } \\
\text { Fuel(lbs) }\end{array}$ & 25.1 & 33.0 & 19.9 & 16.1 \\
\hline $\begin{array}{c}\text { Maneuver Jet } \\
\text { Switches }\end{array}$ & 278 & 4722 & 438 & 310 \\
\hline $\begin{array}{c}\text { Hold Fuel } \\
\text { Rate(lbs/hour) }\end{array}$ & 16.9 & 127.2 & 11.52 & 6.3 \\
\hline $\begin{array}{c}\text { Hold Jet } \\
\text { Switch Rate } \\
\text { (switches/hour) }\end{array}$ & 498.2 & 5878.8 & 559.5 & 483.6 \\
\hline $\begin{array}{c}\text { Average Hold } \\
\text { Eigenangle(deg) }\end{array}$ & 1.30 & .32 & 1.14 & .33 \\
\hline
\end{tabular}

The following figures present Alt Mode results from the rigid orbiter-GRO system. The FCS is still plagued by poor performance during the maneuver due to excessive firings. The change in mass properties seems to have lead to a greater amount of cross coupling between the roll and yaw axis which manifests itself in much more jet chattering, clearly visible in the roll and yaw rate plots for the FCS, Figure 7.57. The fuzzy attitude controller whose fuzzy rules pick jets for firing based upon the error in three axes all at once and attempts to minimize the jet selection angle error does a better job of preventing error correction firings in one axis from driving up the error in another axis and subsequently achieves better limit cycles. 

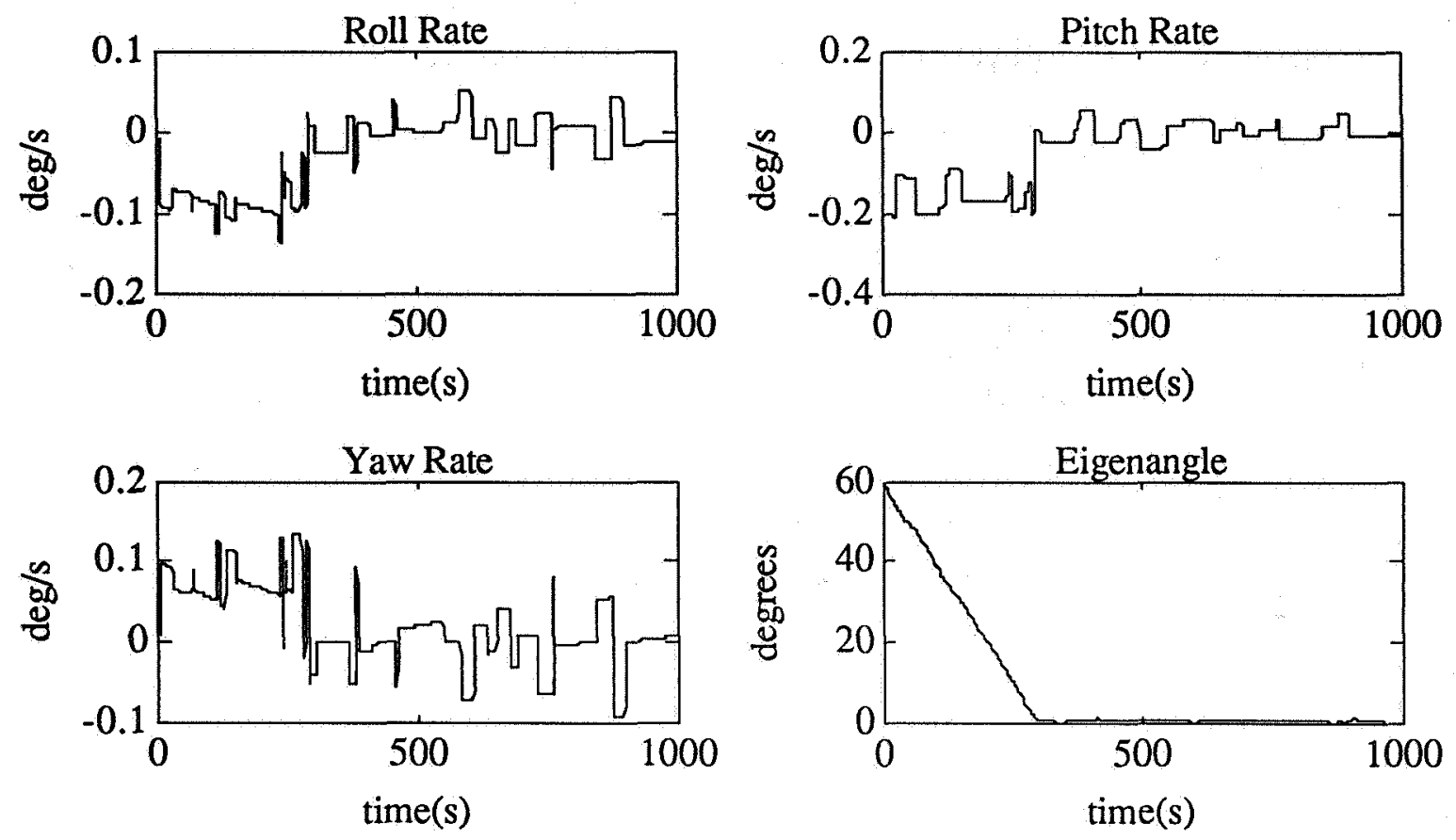

Figure 7.57. FCS Body Rates and Eigenangle for Maneuver C using Alt Mode
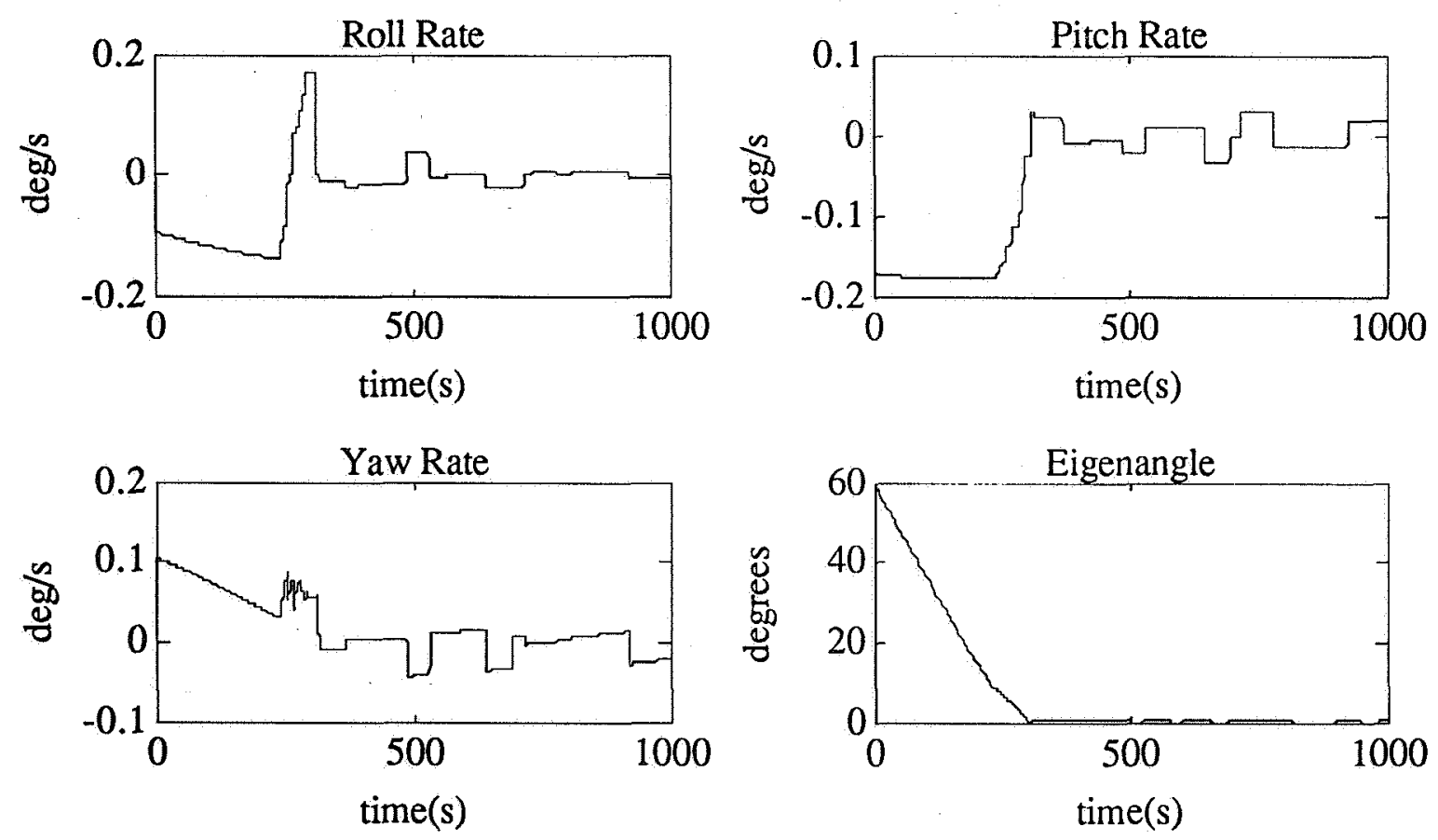

Figure 7.58. Fuzzy Attitude Controller Body Rates and Eigenangle for Maneuver C using Alt Mode 

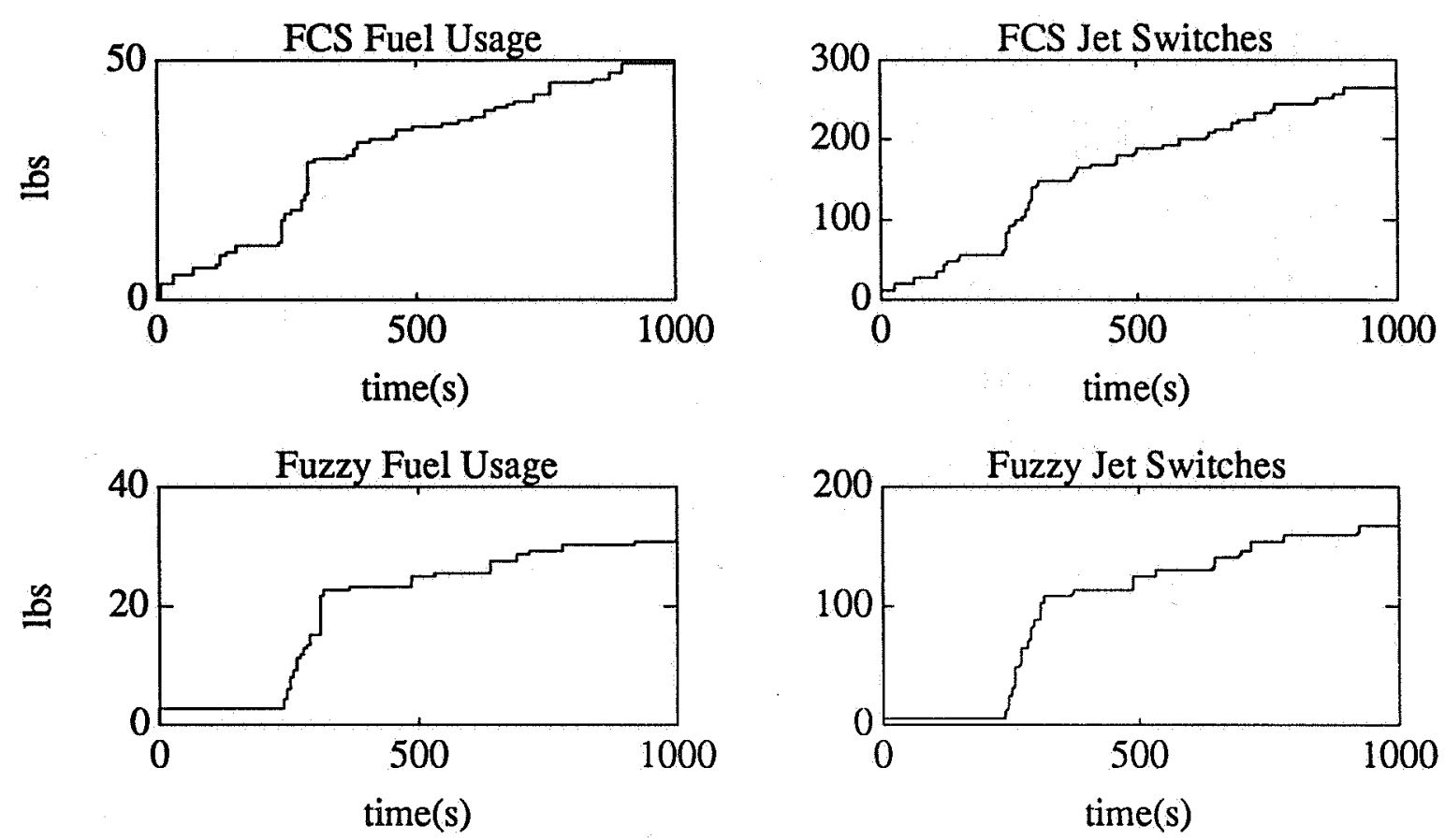

Figure 7.59. Comparison of Fuel and Jet Switches for Maneuver C using Alt Mode

For this case the fuzzy attitude controller had a greater percentage increase in fuel use and jet switches than did the FCS. It should be noted that the fuzzy attitude controller was maintaining $20 \%$ tighter pointing during the attitude hold and still outperformed the FCS for all the quantities measured.

Table 7.11. Comparison of Nominal to Off Nominal Results with Rigid-GRO for the FCS and Fuzzy Attitude Controllers using Alt Mode

\begin{tabular}{|c|c|c|c|c||}
\hline \hline & Nominal FCS & $\begin{array}{c}\text { Off Nominal } \\
\text { FCS }\end{array}$ & $\begin{array}{c}\text { Nominal } \\
\text { Fuzzy }\end{array}$ & $\begin{array}{c}\text { Off Nominal } \\
\text { Fuzzy }\end{array}$ \\
\hline $\begin{array}{c}\text { Maneuver } \\
\text { Fuel(lbs) }\end{array}$ & 18.8 & 29.2 & 11.0 & 22.6 \\
\hline $\begin{array}{c}\text { Maneuver Jet } \\
\text { Switches }\end{array}$ & 86 & 146 & 42 & 108 \\
\hline $\begin{array}{c}\text { Hold Fuel } \\
\text { Rate(lbs/hour) }\end{array}$ & 57.0 & 103.6 & 15.5 & 43.8 \\
\hline $\begin{array}{c}\text { Hold Jet } \\
\text { Switch Rate } \\
\text { (switches/hour) }\end{array}$ & 317.6 & 612.8 & 115.2 & 304.7 \\
\hline $\begin{array}{c}\text { Average Hold } \\
\text { Eigenangle(deg) }\end{array}$ & 3.49 & 1.14 & 2.99 & .93 \\
\hline
\end{tabular}




\subsubsection{Orbiter with GRO, Modeled as Flexible}

Finally, the anti-flex rule package's ability to decrease flexible mode excitation in the presence of modeling uncertainty in the flexible dynamics is examined. First modal amplitudes were increased by $20 \%$ on all modes. Then runs were conducted with the modal frequencies increased and decreased by $20 \%$, respectively.

For verniers, maneuver B was used. Both the FCS controllers using notch filtering and using the rigid rate estimate were run, along with the fuzzy attitude controller, 'msfc', antiflex rules using the slow disturbance rules with jet limiting and anti-chatter rules active. For Alt Mode, maneuver $\mathrm{C}$ was used. Both FCS configurations were run as well as the fuzzy attitude controller, 'ms', anti-flex with slow disturbance rules active.

The expected results are that the FCS runs relying upon the notch filter will have the greatest difficulty. Accurate knowledge of the modal frequencies is required to place the notches. Since knowledge of the modal frequencies was not required to set up the anti-flex rules, their performance should not be adversely affected by the frequency modeling error. The modal amplitude increase should manifest itself as an increase in the 2-norm of the flex rates for all the runs. 
Table 7.12. Flex 2-Norms with and without Modeling Error for Verniers, Maneuver B

\begin{tabular}{||c|c|c|c|}
\hline \hline & Nominal & $\begin{array}{c}\text { Off Nominal } \\
\text { Frequency } \\
\text { Increased }\end{array}$ & $\begin{array}{c}\text { Off Nominal } \\
\text { Frequency } \\
\text { Decreased }\end{array}$ \\
\hline Orig1 & 1.71 & 2.97 & 2.51 \\
\hline Orig2 & 2.00 & .98 & 2.13 \\
\hline msfc & .48 & .53 & .80 \\
\hline
\end{tabular}

Table 7.13. Flex 2-Norms with and without Modeling Error for Alt Mode, Maneuver C

\begin{tabular}{||c|c|c|c||}
\hline & Nominal & $\begin{array}{c}\text { Off Nominal } \\
\text { Frequency } \\
\text { Increased }\end{array}$ & $\begin{array}{c}\text { Off Nominal } \\
\text { Frequency } \\
\text { Decreased }\end{array}$ \\
\hline Orig1 & 2.66 & Unstable & 3.95 \\
\hline Orig2 & 2.60 & 3.85 & 5.25 \\
\hline ms & 1.14 & 1.54 & 2.16 \\
\hline
\end{tabular}

Tables 7.12 and 7.13 show the results for Vernier and Alt Mode respectively. As was expected, both runs using the notch filtering, 'Orig1', faired the worst. Since the modal frequencies were shifted they were no longer attenuated properly by the notch filters. In this specific case with the deployed GRO, the second mode is dominant. This is why the frequency increased runs caused worse results for 'Orig1' than the frequency decreased runs. When the frequency was decreased the dominant mode slid into the notch for the first mode, but when the frequencies were increased the dominant mode went unattenuated and in the case of Alt Mode, drove the FCS unstable. For verniers, performance actually improved for the FCS controller using rigid rate estimate for the higher frequency case. The most likely cause being that the rigid rate estimate improved since the flex mode moved further to the right, becoming further attenuated. This performance improvement was not offset by the larger modal amplitudes.

For both Alt Mode and verniers the anti-flex rule package performed as intended in the presence of modeling error. Since the anti-flex rules do not rely upon frequency information, both were still able to reduce the 2 -norm of the flex rates to levels below that achieved without the anti-flex rules active. 


\section{Chapter 8}

\section{Conclusions and Future Work}

An attitude controller based upon fuzzy rules was successfully developed and tested over a broad range of on-orbit operations using the space shuttle as a test platform. The results demonstrate that the general objectives set forth in the introduction were satisfied. Attitude error can be maintained within specified flight control error margins. In cases of large attitude error (i.e. a maneuver), the error can be removed at a specified rate. These tasks are performed in the presence of disturbances while preventing the excitation of flexible modes, using a reasonable amount of fuel, and limiting the number of jet firings.

The fuzzy attitude controller is easily configured to perform the attitude control objectives on different spacecraft by adjusting a few parameters which are used to size the fuzzy sets employed by the fuzzy controller. This capability was demonstrated by performing different maneuvers and attitude holds with two different actuator systems and two sets of mass properties. The performance of the fuzzy attitude controller proved to be robust to changes in the spacecraft characteristics and mission requirements. In similar exercises using the shuttle flight control system, performance was much more sensitive to changes in pointing requirements or mass properties, for example.

The framework of the fuzzy attitude controller allows easy extensions to address additional objectives. Once an objective is defined for the controller, the controller is extended to 
address it simply by writing an additional collection of rules which are then added to the currently active rules. The linguistic nature of the rules directly reflect the objectives being addressed, allowing intuitive comprehension of the operation of the controller. This is counter to many active spacecraft flight control systems which evolve into a maze of switching logic over many years as "bandaids" are applied to fix problems as they arise. Furthermore, many of the objectives set forth may conflict in some manner. The interpolative nature of the fuzzy rules provide a natural way of making the tradeoff between these conflicting objectives and arriving at a useful solution. The anti-flex rule package is an excellent example. It operates in concert with the baseline or slow trajectory rules. While the later rules are trying to achieve a commanded rate change, the anti-flex rules are promoting selections of jets which will reduce the flex rates. The two objectives may be conflicting, but the end result is that over time both objectives are met.

When comparing the results from simulations using the space shuttle flight control system to the results from the fuzzy attitude controller it becomes apparent that the fuzzy attitude controller avoids many of the pitfalls associated with the traditional, single axis, crisp switching curve techniques utilized by the FCS. The FCS calculates several switching curves as a function of parameters which are hard to characterize for non-symmetric spacecraft. The primary one is control authority. Depending on the direction of rotation this may vary widely. For instance, the control authority in roll for the combined space shuttle-GRO system is much less than for other directions. Utilizing maneuver logic which relies upon accurate knowledge of the control authority to set switching curves will lead to poor performance. The fuzzy rules of the fuzzy attitude controller contain no crisp switch curves, sidestepping this difficulty.

The multi-axis, one step approach used by the fuzzy attitude controller also offers advantages over the single axis, two step approach which first generates jet commands on a per axis basis and then selects jets to fire which best achieve the commands. First, the one 
step decision technique allows rule packages such as the anti-flex package to be more effective. Since the decision of whether to fire or not is based upon the quality of the available jet selection as determined by all the active rule packages, the fuzzy attitude controller may wait to correct small attitude errors until the flex rates have reversed direction and a jet selection is available which looks better from the anti-flex perspective. This capability would be very difficult to mimic within the confines of the FCS which first decides to fire and then tries to find appropriate jets to achieve the commanded rate change. Second, the fuzzy attitude controller does not include the assumption of no inter-axis coupling during rate changes. This prevents firing patterns where the error in one axis causes a firing which drives the error in another axis beyond its crisp deadband causing yet another firing, and potentially many more.

Based upon the results presented in Chapter 7 some simple recommendations can be made for appropriately configuring which rule packages are active and their relative output weighting. First, unless operations require extremely precise pointing, for which the baseline rules are quite good, or prevent an accurate slow disturbance acceleration estimate, the slow disturbance rule package should be active instead of the baseline rules. This dramatically reduces the number of jet duty cycles. In deciding whether, or how much to, employ the jet limiting and anti-chatter rules it is important to consider how many control system sample intervals will occur during a typical burn. The greater this quantity, the more beneficial these rules will be and thus they should be weighted more heavily. If there are very few control system sample intervals during a typical burn, it is better to weight these rules very little or none at all, allowing the controller greater freedom to choose a jet selection which maximizes acceleration in the direction of the commanded rate change while minimizing the angle error (In this case the number of jet switches will already be small precluding much of the need for the anti-chatter rule package anyway). If operations require reducing the excitation of flexible modes, the anti-flex rule package should be used, weighted more heavily for systems with greater number of control system sample intervals 
per burn. Following these guidelines, to attain best performance for shuttle operations with verniers the 'sfc' setup, table 7.4, should be used. For Alt Mode only the slow disturbance rules should be used.

\subsection{Recommendations for Future Work}

The next logical step for future work is to extend the three degree of freedom fuzzy attitude controller to six degrees of freedom, adding the capability to control translation. Then the possibility of codifying the expert knowledge used by pilots during on-orbit proximity operations into fuzzy rules should be investigated. For example, space shuttle pilots follow a well defined set of rules to conduct approaches to other orbiting spacecraft. Perhaps performance could be improved if these rules were automated through fuzzy logic, allowing control action decisions to be made based upon more complete and accurate state information than is presented to, or perceived by, the human pilot.

In Chapter 4 all the fuzzy sets were defined using simple trapezoidal membership functions for their simplicity and ease of implementation. Future work may show a benefit to using more complicated shapes. The shaping of membership functions could be used to control the rate of transition from one fuzzy set to another. For example, in some situations it may be beneficial to begin the transition gradually, followed by an increasing rate of transition . In this case, a semi-bell shaped membership function might be applicable. This could be useful for spacecraft applications with large values for MAXJETS. Instead of incurring a linearly increasing penalty for jet selection with greater number of jets on, the penalty could be greater, for example, when changing from a three jet on selection to a four jet on selection, than when changing from a two to three jet on selection.

The domains of the inputs to the fuzzy rules discussed in this thesis were generally divided into two fuzzy sets, big and small. Future research may include evaluation of the possible performance improvement achievable by increasing the number of state space divisions to 
include such fuzzy sets as medium, very big, or very small. This increases the interpolative quality of the fuzzy logic at the expense of increasing computational requirements. Clearly there is a tradeoff to be made.

The performance of the jet anti-chatter rule package may be improved if a new parameter, duration, were added to the predicate of the rules. The duration quantity could reflect how long jets had been firing consecutively. As this duration quantity increased it would decrease the benefit seen in the anti-chatter rule package output from not changing the selected jets. Another possible metric to vary the penalty associated with the anti-chatter rule package would be a measure of how much the commanded rate change has shifted in direction. If it has changed direction by a large amount then the penalty associated with switching jets should be reduced.

The baseline rule package determines commanded eigenangle rate independent of the available jet selections. Some benefit may be realized if this initial step could be incorporated into the jet selection rules. Problems arise because the search space would be increased to include combinations of jets at different commanded rate changes. This approach would also lead to an indeterminate maneuver completion time which may be unacceptable to mission planning and operations. 



\section{References}

[1] Zadeh, L., "Fuzzy Sets," Information and Control, vol. 8, pp. 338-353, 1965.

[2] Lea, R.N. and Jani, Y., "Spacecraft Attitude Control System Based on Fuzzy Logic Principles," Proceedings of ROBEX'89, 1989.

[4] Berenji, H. R., Jani, Y., and Lea, R.N., "Approximate Reasoning-Based Learning and Control for Proximity Operations and Docking in Space," Proceedings of the AIAA Guidance and Control Conference, Volume 3, August 12-14, 1991

[5] Stephanides, S., "Controls Applications of Fuzzy Set Theory," Masters Thesis, MIT, Cambridge, MA, CSDL-T-1124, June 1992

[6] Chen, Y. and Tsao, T., "A New Approach for the Global Analysis of Fuzzy Dynamical Systems", Proceedings of the 27th Conference on Decision and Control, IEEE 88CH2531-2/88/0000-1415, 1988

[7] Chen, T., "Stability Analysis of Fuzzy Control -- A Lyapunov Approach", IEEE CH2503-1/87/0000-1027, 1987

[8] Kaplan, M. H., Modern Spacecraft Dynamics \& Control, John Wiley \& Sons, NY, 1976

[9] Carroll, J. V., "The Notation and Use of Quaternions for Shuttle Ascent Steering," CSDL Memo 10C-75-47, 1975

[10] Greenwood, D. T., Principles of Dynamics, Second Edition, pp. 337-339, Prentice Hall, NJ, 1987.

[11] Bergmann, E. V., "A New Spacecraft Autopilot", Masters Thesis, Dept. of Aero. and Astro., MIT, 1976

[12] Athans, M., "Course Notes in Multivariable Control Systems, I \& II", MIT, 1991

[13] Zimpfer, D., "IOS/ICDS Flight Control and Environment Verification Cases", CSDL Memorandum DJZ-91-06 Task 75, Nov. 15, 1991

[14] Moore, D. E., "Payload Information Document, Remote Manipulator System", JSC21000-INF-RMS, 1986

[15] Hattis, P., "A Review of the Space Shuttle Orbital Flight Control System", CSDL-P1786,1983 
[16] Appleby, B., "Acceleration Estimation and Their Effect on Shuttle Performance", CSDL Memo Number: SSV-87-20, 1987

[17] Barrows, T. M., "A Simplified Simulation for the Analysis of Heavy Payloads on the Shuttle Remote Manipulator Arm," CSDL-R-1730, September 1984

[18] Phillips, M., "Space Shuttle Orbiter Operational Level C, Functional Subsystem Software Requirements Guidance, Navigation, and Control, Part C, Flight Control, Orbit DAP", Rockwell International, STS83-0009E OI-22, 1991 